Supporting Information

\title{
Exploring the Effects of Node Topology, Connectivity, and Metal Identity on the Binding of Nerve Agents and Their Hydrolysis Products in Metal- Organic Frameworks
}

\author{
Matthew L. Mendonca,$^{\dagger}$ Debmalya Ray ${ }^{\ddagger}$ Christopher J. Cramer, ${ }^{\ddagger}$ and Randall Q. Snurr ${ }^{*} \dagger$ \\ †Department of Chemical \& Biological Engineering, Northwestern University, 2145 Sheridan \\ Road, Evanston, IL 60208, United States \\ Department of Chemistry, Chemical Theory Center and Minnesota Supercomputing Institute, \\ University of Minnesota, 207 Pleasant Street Southeast, Minneapolis, Minnesota 55455, United \\ States
}

${ }^{*}$ Corresponding Author E-mail: snurr@northwestern.edu

\section{Computational Details: Periodic Calculations}

For the periodic DFT calculations, we tested the convergence of total electronic energy for the unit cell of Zr-mono-defect UiO-66 with respect to the plane-wave kinetic energy cutoff and k-point grid used in the calculation. As shown in Table S0a, the electronic energies are converged to $0.0001 \mathrm{eV} /$ atom. Thus, for all periodic structural optimizations, we used a plane-wave kinetic energy cutoff of $520 \mathrm{eV}$ and $\Gamma$-point sampling of the Brillouin zone.

Table S0a. Convergence test of total electronic energy for the periodic structure of Zr-mono-defect UiO66 with respect to plane-wave kinetic energy cutoff and k-point grid.

\begin{tabular}{|c|c|c|c|}
\hline $\begin{array}{c}\text { Plane-wave Kinetic } \\
\text { Energy Cutoff (eV) }\end{array}$ & k-point Grid & $\begin{array}{c}\text { Total Electronic } \\
\text { Energy (eV) }\end{array}$ & $\begin{array}{c}\text { Change in Electronic } \\
\text { Energy/Atom (eV/atom) }\end{array}$ \\
\hline 500 & $1 \times 1 \times 1$ & -1648.98021708 & -0.0005 \\
\hline 510 & $1 \times 1 \times 1$ & -1649.03621503 & -0.0002 \\
\hline 520 & $1 \times 1 \times 1$ & -1649.09361362 & 0.0000 \\
\hline 530 & $1 \times 1 \times 1$ & -1649.09777242 & 0.0000 \\
\hline 540 & $1 \times 1 \times 1$ & -1649.11978393 & 0.0001 \\
\hline 520 & $2 \times 2 \times 2$ & -1649.11760800 & 0.0001 \\
\hline
\end{tabular}

\section{Computational Details: Cluster Model Calculations}

For all cluster model DFT calculations discussed in this section, the SDD basis set and its associated effective core potential was used for metal atoms of the nodes. As described in the main text, we used the M06-L/def2-SVP level of theory to perform gas phase geometry optimizations and frequency calculations. We chose the M06-L density functional for geometry optimizations because it exhibits a reasonable balance between chemical accuracy and computational expense, it inherently includes some dispersion corrections, ${ }^{1}$ it performs well for transition-metal systems, ${ }^{2}$ and it has provided reliable results in previous computational studies involving MOF-catalyzed nerve agent hydrolysis. ${ }^{3-6}$ To refine the electronic energies, we then performed single point 
calculations with the M06-2X/def2-SVP level of theory on the gas phase optimized geometries, using the SMD continuum solvation model with parameters for water $(\varepsilon=78.355)$. All reported Gibbs free energies (at 298.15 K and $1 \mathrm{~atm}$ ) were computed by combining M06-2X(SMD)/def2SVP single point energies with thermochemical contributions obtained at the M06-L(gas phase)/def2-SVP level of theory.

To test the influence of the computational protocol, we performed additional SMD single point calculations at different levels of theory, using the M06-L gas phase optimized geometries. Specifically, we tested the B3LYP-D3(BJ) ${ }^{7-11}$ and $\omega-B 97 X-D^{12}$ density functionals as well as the larger def2-TZVP basis set. ${ }^{13} \mathrm{We}$ performed two orthogonal comparisons to evaluate the relative trends in binding free energies between molecules and across MOF nodes. First, we selected one MOF node site (Zr-NU-1000 (large pore)) and compared the trends in binding free energies for two molecules ( $\mathbf{6}$ and 18) on this node, where we used the most favorable molecular orientation on the node for each molecule (listed in Table S1). Second, we selected one molecule (6) and compared the trends in binding free energies for this molecule on two different node sites (Zr-NU1000 (large pore) and Zr-bi(trans)-defect UiO-66), again using the most favorable molecular orientation on each node.

As shown in Table S0b, SMD single point calculations performed at the B3LYP-D3(BJ)/def2SVP, $\omega$-B97X-D/def2-SVP, and M06-2X/def2-TZVP levels of theory provided the same trends in binding free energies between molecules and across MOF nodes as those reported for M062X/def2-SVP. This limited variation in results suggests little sensitivity to the choice of density functional and basis set, although the absolute binding free energies of specific systems understandably show more variability. For example, the more negative values obtained with B3LYP-D3(BJ) are most likely associated with the D3(BJ) correction overestimating dispersion contributions in such crowded node environments. ${ }^{14}$ Thus, all reported free energies in the main text and in the remainder of the Supporting Information correspond to those computed by combining M06-2X(SMD)/def2-SVP single point energies with thermochemical contributions obtained at the M06-L(gas phase)/def2-SVP level of theory.

Table S0b. Binding free energies (in $\mathrm{kJ} / \mathrm{mol}$ ) computed by performing SMD single point calculations at different levels of theory, using the M06-L gas phase optimized geometries.

\begin{tabular}{|c|c|c|c|c|}
\hline Node/Molecule & $\begin{array}{c}\text { M06-2X/ } \\
\text { def2-SVP }\end{array}$ & $\begin{array}{c}\text { B3LYP-D3(BJ)/ } \\
\text { def2-SVP }\end{array}$ & $\begin{array}{c}\boldsymbol{\omega} \text {-B97X-D/ } \\
\text { def2-SVP }\end{array}$ & $\begin{array}{c}\text { M06-2X/ } \\
\text { def2-TZVP }\end{array}$ \\
\hline Zr-NU-1000 (large pore)/6 & -2.7 & -20.1 & 1.0 & 31.3 \\
\hline Zr-NU-1000 (large pore)/18 & -18.7 & -42.6 & -20.0 & 28.5 \\
\hline Zr-bi(trans)-defect UiO-66/6 & -17.0 & -37.0 & -16.7 & 15.1 \\
\hline
\end{tabular}




\section{Hypothetical Hydrolysis Pathways on M(IV)-MOF Nodes}
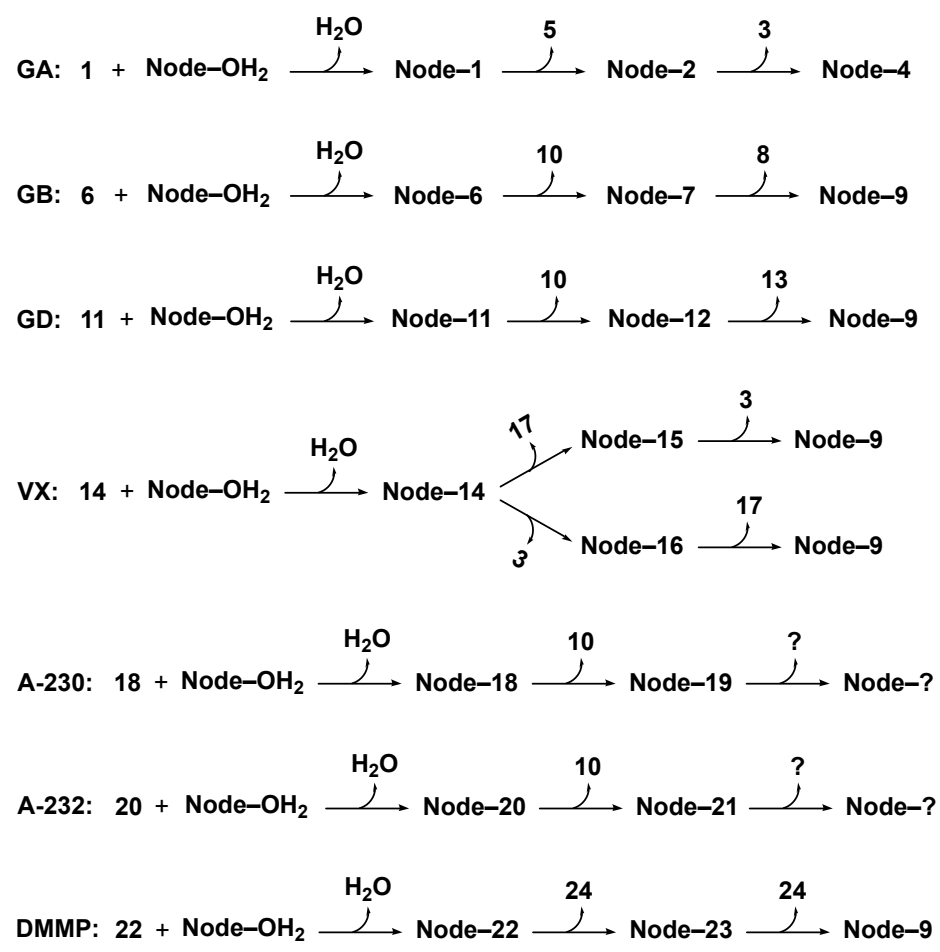

Figure S1. Hypothetical hydrolysis pathways for the nerve agent and simulant molecules occurring on M(IV)-MOF nodes, using the molecule numbering established in Figure 1a of the main text. The terminology "Node-N" represents molecule $\mathbf{N}$ bound to the node. Numbers listed above the curved reaction arrows represent hydrolysis product molecules (monodentate anions, alcohols, and thiols) released into solution. The question marks in the pathways for A-230 and A-232 indicate that the full hydrolysis mechanisms for these Novichok agents are currently unknown. 


\section{Representative Binding Modes}

In Figure S2a, the node-noOH $\mathrm{H}_{2}$ structure represents a bare node where the terminal $-\mathrm{OH}_{2}$ group has been removed from the active (binding) site, which was used in the formula to calculate the binding energies of neutral molecules throughout the study. In Figure S2b, the node-noOH structure represents a bare node where the terminal $-\mathrm{OH}$ group has been removed from the binding site, which was used in the formula to calculate the binding energies of monodentate anions. In Figure S2c, the node-noOH, $\mathrm{OH}$ structure represents a bare node where the terminal $-\mathrm{OH}_{2}$ and $\mathrm{OH}$ groups have been removed from the binding site, which was used in the formula to calculate the binding energies of bidentate anions.

(a)

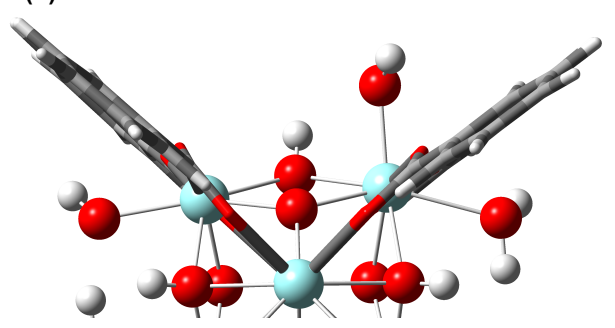

(b)

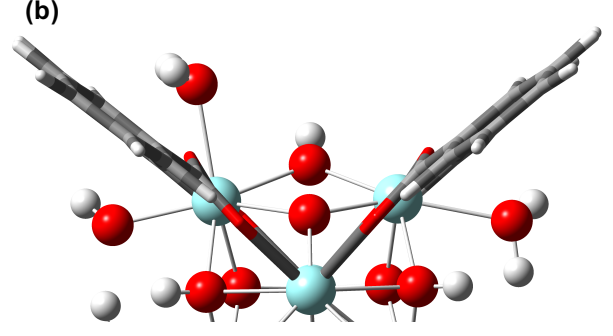

(c)

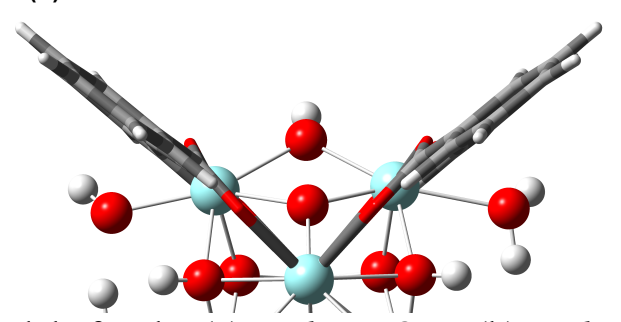

Figure S2. Optimized cluster models for the (a) node-noOH $\mathrm{H}_{2}$, (b) node-noOH, and (c) node-noOH, $\mathrm{OH}$ bare node structures of Zr-NU-1000 (large pore). The benzoate linkers around the binding site are shown in tube format for clarity, and only the top half of the node is shown to highlight the site where binding occurs. Dark gray, white, red, and turquoise spheres represent $\mathrm{C}, \mathrm{H}, \mathrm{O}$, and $\mathrm{Zr}$ atoms, respectively. 
Figure S3 shows structures that are representative of the binding modes for (a) nerve agent and simulant molecules, (b) alcohol and thiol product molecules, (c) bidentate anions, and (d) monodentate anions.
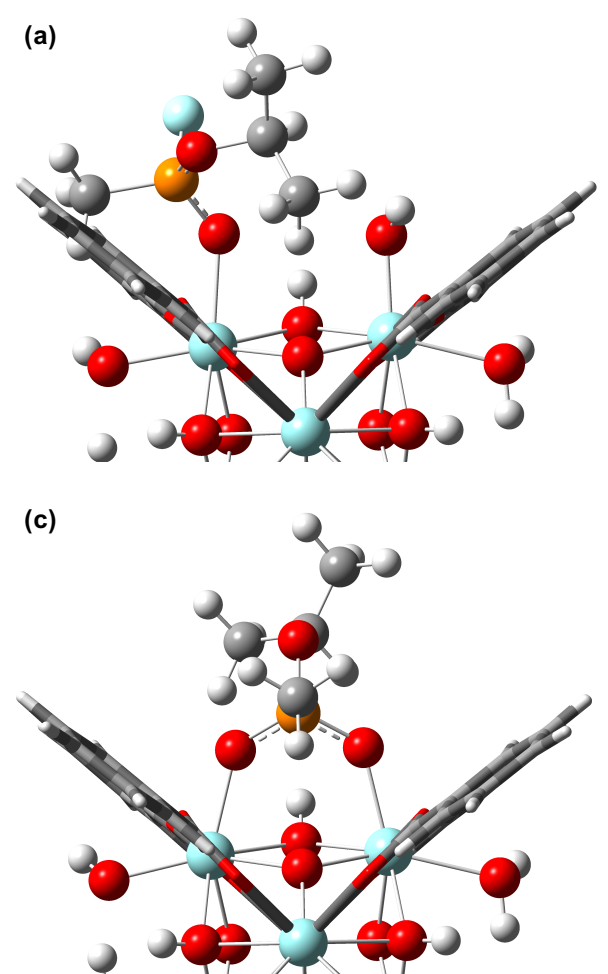

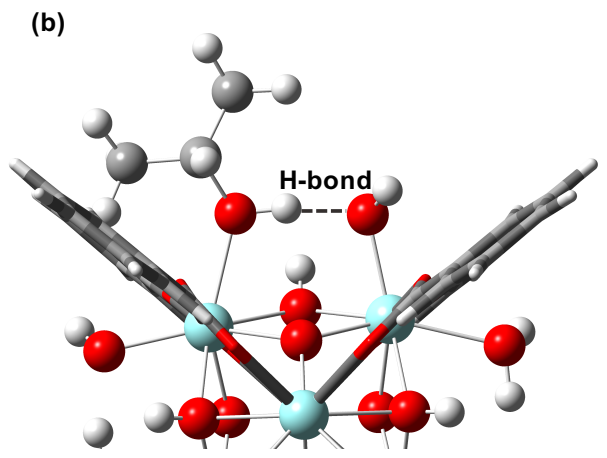

(d)

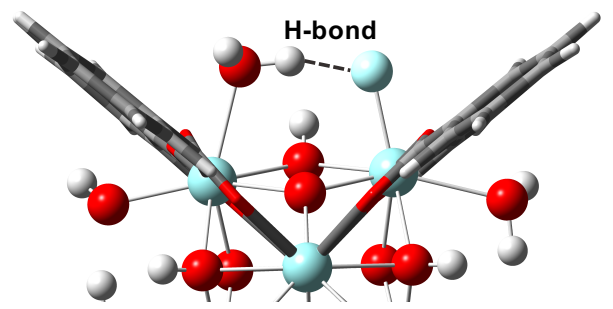

Figure S3. Optimized cluster models for molecules (a) 6 (sarin, GB), (b) 8 (isopropanol), (c) 7 (isopropyl methylphosphonic acid, IMPA), and (d) $\mathbf{1 0}$ (fluoride anion) bound to the node of Zr-NU-1000 (large pore). The benzoate linkers around the binding site are shown in tube format for clarity, and only the top half of the node is shown to highlight the site where binding occurs. Dark gray, white, red, orange, light blue, and turquoise spheres represent $\mathrm{C}, \mathrm{H}, \mathrm{O}, \mathrm{P}, \mathrm{F}$, and $\mathrm{Zr}$ atoms, respectively.

\section{Relative Stabilities of Different Binding Orientations: Nerve Agent/Simulant Molecules}
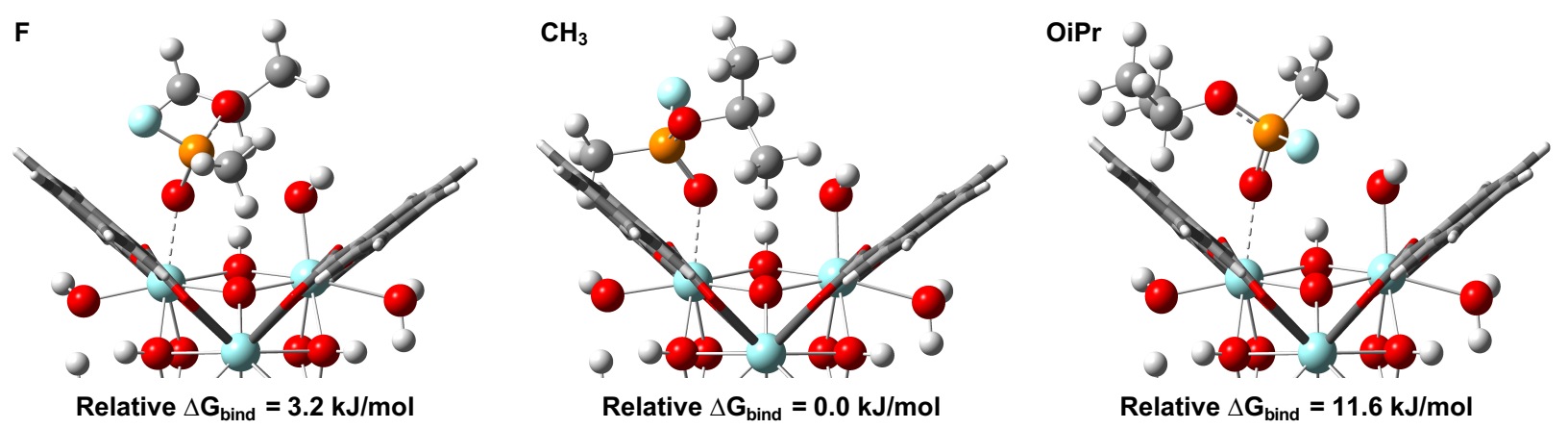

Figure S4. Optimized cluster models for the three possible orientations of GB (6) bound to the node of ZrNU-1000 (large pore), along with their relative binding free energies. Each orientation is named according to the $\mathrm{R}$ group $\left(\mathrm{F}, \mathrm{CH}_{3}\right.$, or $\left.\mathrm{OiPr}\right)$ that is approximately collinear with the neighboring terminal node $\mathrm{Zr}-\mathrm{OH}$ group. The benzoate linkers around the binding site are shown in tube format for clarity, and only the top half of the node is shown to highlight the site where binding occurs. Dark gray, white, red, orange, light blue, and turquoise spheres represent $\mathrm{C}, \mathrm{H}, \mathrm{O}, \mathrm{P}, \mathrm{F}$, and $\mathrm{Zr}$ atoms, respectively. 
Table S1. Relative binding free energies (in $\mathrm{kJ} / \mathrm{mol}$ ) for different orientations of nerve agent and simulant molecules bound to $\mathrm{Zr}(\mathrm{IV})$-MOF node sites. Values in red indicate the most favorable molecular orientation for each node site.

\begin{tabular}{|c|c|c|c|c|c|c|c|}
\hline Molecule & Orientation & MOF-808 & $\begin{array}{c}\text { NU-1000 } \\
\text { (large pore) }\end{array}$ & $\begin{array}{c}\text { bi(trans)-defect } \\
\text { UiO-66 }\end{array}$ & $\begin{array}{c}\text { mono-defect } \\
\text { UiO-66 }\end{array}$ & $\begin{array}{c}\text { bi(cis)-defect } \\
\text { UiO-66 }\end{array}$ & $\begin{array}{l}\text { NU-1000 } \\
\text { (c pore) }\end{array}$ \\
\hline \multirow{3}{*}{1} & OEt & 0.0 & 4.6 & 0.0 & 14.8 & 30.8 & 3.1 \\
\hline & $\mathrm{NMe}_{2}$ & 7.3 & 0.0 & 4.2 & 0.0 & 0.0 & 0.0 \\
\hline & $\mathrm{CN}$ & 43.6 & 12.5 & 11.6 & 18.8 & 24.2 & 3.4 \\
\hline \multirow{3}{*}{6} & OiPr & 0.0 & 11.6 & 29.7 & 12.6 & 0.4 & 17.7 \\
\hline & $\mathrm{CH}_{3}$ & 29.5 & 0.0 & 0.0 & 0.0 & 14.8 & 19.7 \\
\hline & $\mathrm{F}$ & 39.5 & 3.2 & 12.5 & 4.4 & 0.0 & 0.0 \\
\hline \multirow{3}{*}{11} & OR & 0.0 & 16.8 & 9.3 & 5.3 & 0.0 & 0.0 \\
\hline & $\mathrm{CH}_{3}$ & 21.9 & 0.0 & 0.0 & 3.8 & 2.9 & 35.2 \\
\hline & $\mathrm{F}$ & 53.4 & 19.0 & 18.3 & 0.0 & 18.1 & 6.6 \\
\hline \multirow{3}{*}{14} & $\mathrm{CH}_{3}$ & 0.0 & 0.0 & 0.0 & 0.0 & 0.0 & 6.8 \\
\hline & SR & 2.4 & 30.4 & 68.6 & 16.4 & 20.9 & 0.0 \\
\hline & $\mathrm{OEt}$ & 5.8 & 44.8 & $\mathrm{~N} / \mathrm{A}^{a}$ & 17.9 & 13.4 & 11.8 \\
\hline \multirow{3}{*}{18} & $\mathrm{CH}_{3}$ & 0.0 & 0.0 & 1.3 & 0.0 & 0.0 & 10.3 \\
\hline & NR & 9.2 & 14.9 & 19.1 & 1.9 & 23.9 & 25.2 \\
\hline & $\mathrm{F}$ & 20.3 & 4.9 & 0.0 & 3.9 & 7.2 & 0.0 \\
\hline \multirow{3}{*}{20} & $\mathrm{~F}$ & 0.0 & 23.8 & 19.3 & 21.2 & 0.0 & 12.8 \\
\hline & NR & 6.2 & 12.4 & 24.2 & 20.3 & 1.6 & 39.0 \\
\hline & $\mathrm{OMe}$ & 19.1 & 0.0 & 0.0 & 0.0 & 12.3 & 0.0 \\
\hline \multirow{2}{*}{22} & $\mathrm{OMe}$ & 0.0 & 0.0 & 7.3 & 0.0 & 0.0 & 0.9 \\
\hline & $\mathrm{CH} 3$ & 10.7 & 14.5 & 0.0 & 9.6 & 28.1 & 0.0 \\
\hline
\end{tabular}

${ }^{a} \mathrm{~N} / \mathrm{A}$ indicates that the OEt orientation for molecule $\mathbf{1 4}$ could not be optimized on the Zr-bi(trans)-defect UiO-66 node, despite multiple attempts. 


\section{Effects of Molecular Size and Dispersion on Binding Energy: Neutral Molecules}

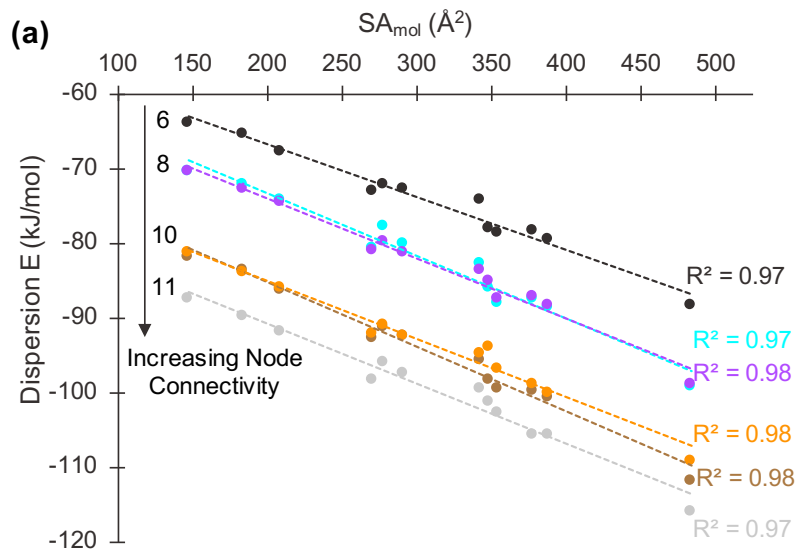

Zr-MOF-808

Zr-NU-1000 (large pore)

Zr-bi(trans)-defect UiO-66 (b)

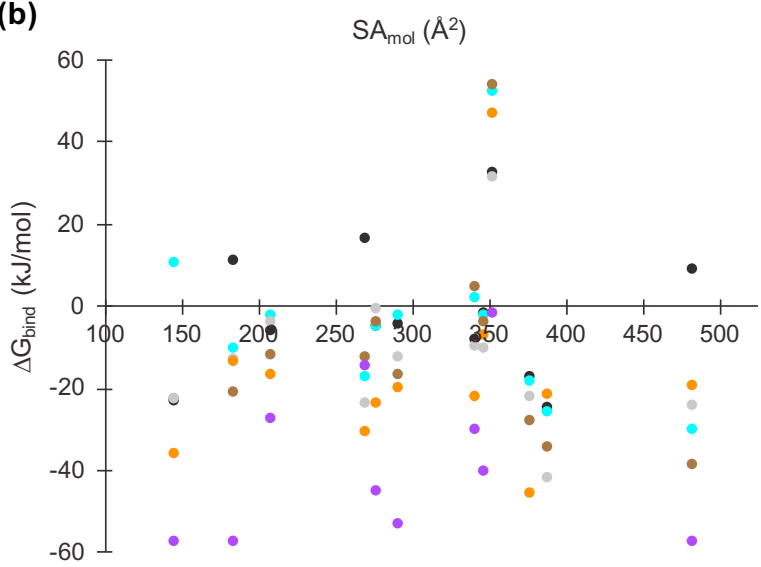

Zr-mono-defect UiO-66

Zr-bi(cis)-defect UiO-66

$\mathrm{Zr}-\mathrm{NU}-1000$ (c pore)

Figure S5. The effect of molecular solvent-accessible surface area $\left(S A_{m o l}\right)$ on (a) the dispersion energy and (b) the binding free energy computed for node-bound neutral molecules. Neutral molecules refer to molecules 1, 3, 6, 8, 11, 13, 14, 17, 18, 20, 22, 24, and $\mathrm{H}_{2} \mathrm{O}$.

\section{Relative Stabilities of Different Binding Orientations: Bidentate Anions}
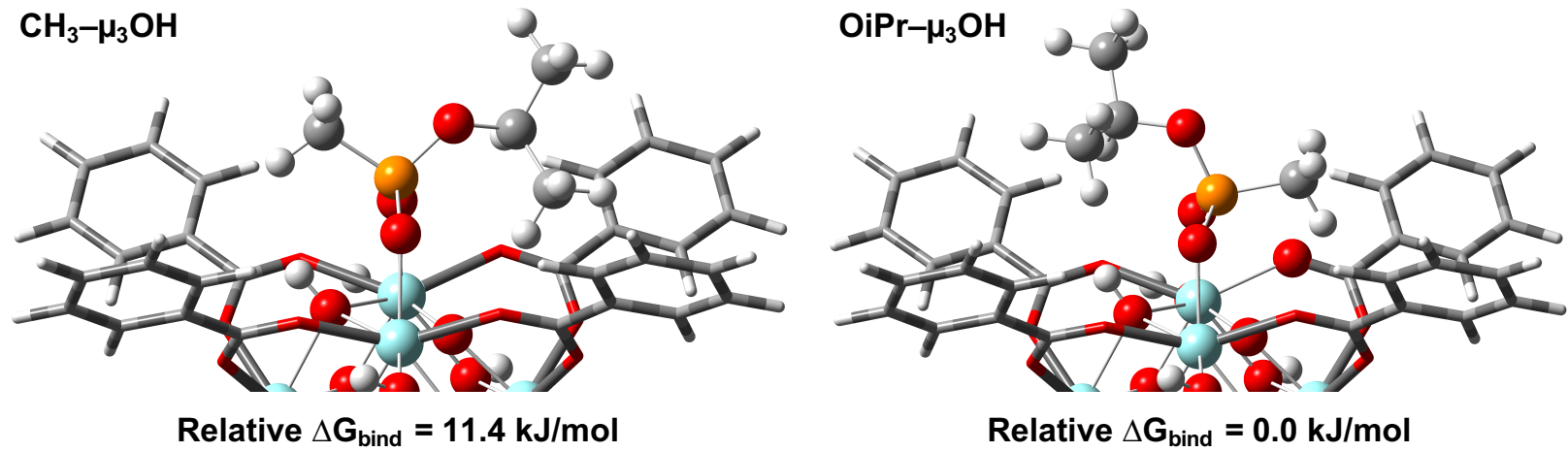

Figure S6. Optimized cluster models for the two possible orientations of IMPA (7) bound to the node of Zr-NU-1000 (large pore), along with their relative binding free energies. Each orientation is named according to the $\mathrm{R}$ group $\left(\mathrm{CH}_{3}\right.$ or $\left.\mathrm{OiPr}\right)$ that is directed towards the bridging node hydroxyl group at the binding site. The benzoate linkers around the binding site are shown in tube format for clarity, and only the top half of the node is shown to highlight the site where binding occurs. Dark gray, white, red, orange, and turquoise spheres represent $\mathrm{C}, \mathrm{H}, \mathrm{O}, \mathrm{P}$, and $\mathrm{Zr}$ atoms, respectively. 
Table S2. Relative binding free energies (in $\mathrm{kJ} / \mathrm{mol}$ ) for different orientations of bidentate anion products bound to $\mathrm{Zr}(\mathrm{IV})-\mathrm{MOF}$ node sites. Values in green indicate the most favorable orientation for each node site.

\begin{tabular}{|c|c|c|c|c|c|c|c|}
\hline Molecule & Orientation & MOF-808 & $\begin{array}{l}\text { NU-1000 } \\
\text { (large pore) }\end{array}$ & $\begin{array}{l}\text { bi(trans)-defect } \\
\text { UiO-66 }\end{array}$ & $\begin{array}{l}\text { mono-defect } \\
\text { UiO-66 }\end{array}$ & $\begin{array}{l}\text { bi(cis)-defect } \\
\text { UiO-66 }\end{array}$ & $\begin{array}{c}\text { NU-1000 } \\
\text { (c pore) }\end{array}$ \\
\hline \multirow{2}{*}{2} & $\mathrm{NMe}_{2}-\mu_{3} \mathrm{OH}$ & 0.0 & 0.0 & 0.0 & 9.1 & 12.8 & 0.0 \\
\hline & $\mathrm{OEt}-\mu_{3} \mathrm{OH}$ & 12.9 & 14.5 & 5.1 & 0.0 & 0.0 & 7.8 \\
\hline \multirow{2}{*}{4} & $\mathrm{NMe}_{2}-\mu_{3} \mathrm{OH}$ & 0.0 & 4.0 & 3.4 & 0.0 & 0.0 & 8.7 \\
\hline & $\mathrm{OH}-\mu_{3} \mathrm{OH}$ & 4.2 & 0.0 & 0.0 & 1.7 & 7.1 & 0.0 \\
\hline \multirow{2}{*}{7} & $\mathrm{CH}_{3}-\mu_{3} \mathrm{OH}$ & 0.0 & 11.4 & 0.1 & 11.6 & 3.7 & 0.0 \\
\hline & $\mathrm{OiPr}-\mu_{3} \mathrm{OH}$ & 23.9 & 0.0 & 0.0 & 0.0 & 0.0 & 4.5 \\
\hline \multirow{2}{*}{9} & $\mathrm{CH}_{3}-\mu_{3} \mathrm{OH}$ & 0.0 & 0.0 & 0.5 & 0.2 & 0.0 & 0.0 \\
\hline & $\mathrm{OH}-\mu_{3} \mathrm{OH}$ & 9.4 & 8.9 & 0.0 & 0.0 & 10.8 & 0.2 \\
\hline \multirow{2}{*}{12} & $\mathrm{CH}_{3}-\mu_{3} \mathrm{OH}$ & 0.0 & 10.8 & 4.4 & 11.0 & 11.6 & 3.5 \\
\hline & $\mathrm{OR}-\mu_{3} \mathrm{OH}$ & 7.6 & 0.0 & 0.0 & 0.0 & 0.0 & 0.0 \\
\hline \multirow{2}{*}{15} & $\mathrm{CH}_{3}-\mu_{3} \mathrm{OH}$ & 0.0 & 0.0 & 0.03 & 2.0 & 0.0 & 0.0 \\
\hline & $\mathrm{OEt}-\mu_{3} \mathrm{OH}$ & 30.0 & 35.8 & 0.0 & 0.0 & 2.8 & 0.4 \\
\hline \multirow{2}{*}{16} & $\mathrm{CH}_{3}-\mu_{3} \mathrm{OH}$ & 0.0 & 24.3 & 16.7 & 11.1 & 5.1 & 15.5 \\
\hline & $\mathrm{SR}-\mu_{3} \mathrm{OH}$ & 11.0 & 0.0 & 0.0 & 0.0 & 0.0 & 0.0 \\
\hline \multirow{2}{*}{19} & $\mathrm{CH}_{3}-\mu_{3} \mathrm{OH}$ & 0.0 & 0.0 & 0.2 & 5.1 & 24.9 & 4.5 \\
\hline & $\mathrm{NR}-\mu_{3} \mathrm{OH}$ & 24.8 & 6.6 & 0.0 & 0.0 & 0.0 & 0.0 \\
\hline \multirow{2}{*}{21} & $\mathrm{OMe}-\mu_{3} \mathrm{OH}$ & 0.0 & 0.0 & 0.0 & 0.0 & 9.1 & 0.0 \\
\hline & $\mathrm{NR}-\mu_{3} \mathrm{OH}$ & 17.0 & 7.8 & 5.6 & 26.5 & 0.0 & 1.0 \\
\hline \multirow{2}{*}{23} & $\mathrm{CH}_{3}-\mu_{3} \mathrm{OH}$ & 0.0 & 10.9 & 1.9 & 12.2 & 4.3 & 3.4 \\
\hline & $\mathrm{OMe}-\mu_{3} \mathrm{OH}$ & 4.8 & 0.0 & 0.0 & 0.0 & 0.0 & 0.0 \\
\hline
\end{tabular}




\section{Effects of Molecular Size and Dispersion on Binding Energy: Bidentate Anions}

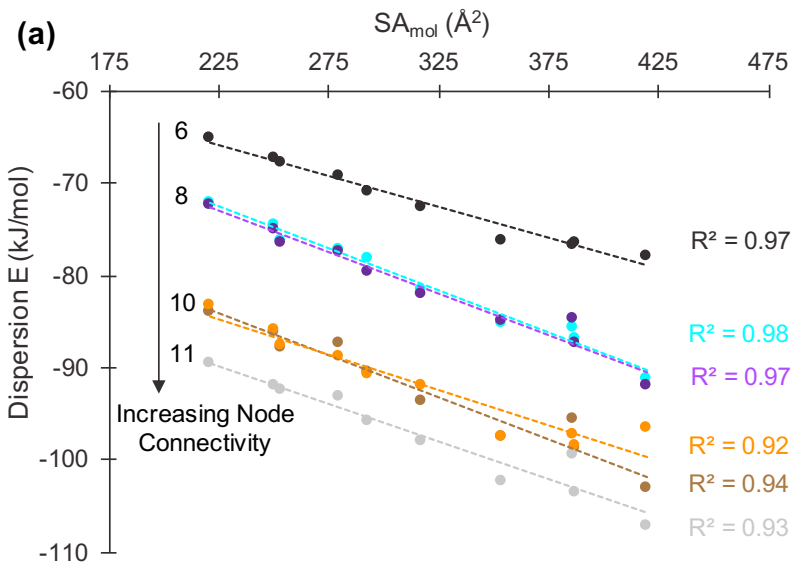

Zr-MOF-808

$\mathrm{Zr}-\mathrm{NU}-1000$ (large pore) Zr-bi(trans)-defect UiO-66

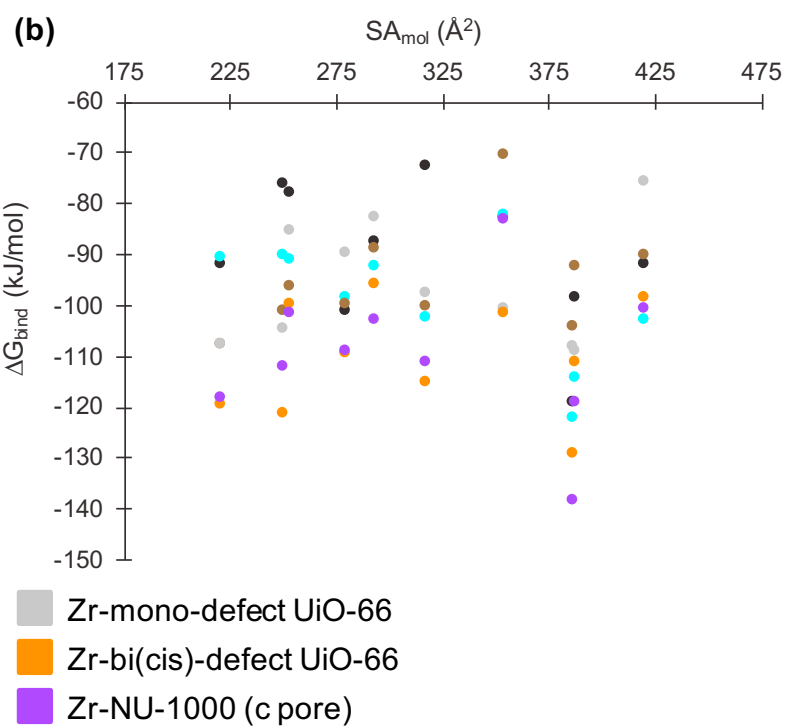

Figure S7. The effect of molecular solvent-accessible surface area $\left(S A_{m o l}\right)$ on (a) the dispersion energy and (b) the binding free energy computed for node-bound bidentate anions. Bidentate anions refer to molecules $2,4,7,9,12,15,16,19,21$, and 23. 


\section{Binding Free Energy Values for All Molecules: Zr(IV)-MOF Nodes}

Table S3. Binding free energies (in $\mathrm{kJ} / \mathrm{mol}$ ) for molecules bound to $\mathrm{Zr}(\mathrm{IV})-\mathrm{MOF}$ node sites in their most favorable orientations, corresponding to results in Figure 2. Negative and positive values indicate favorable and unfavorable binding, respectively.

\begin{tabular}{|c|c|c|c|c|c|c|}
\hline Molecule & MOF-808 & $\begin{array}{c}\text { NU-1000 } \\
\text { (large pore) }\end{array}$ & $\begin{array}{c}\text { bi(trans)-defect } \\
\text { UiO-66 }\end{array}$ & $\begin{array}{c}\text { mono-defect } \\
\text { UiO-66 }\end{array}$ & $\begin{array}{c}\text { bi(cis)-defect } \\
\text { UiO-66 }\end{array}$ & $\begin{array}{l}\text { NU-1000 } \\
\text { (c pore) }\end{array}$ \\
\hline $\mathrm{H}_{2} \mathrm{O}$ & -7.9 & -28.1 & -29.9 & -40.9 & -54.5 & -61.1 \\
\hline 1 & -8.5 & 1.9 & 4.6 & -10.0 & -22.6 & -30.3 \\
\hline 2 & -72.9 & -102.6 & -100.4 & -97.9 & -115.4 & -111.6 \\
\hline 3 & 10.8 & -10.8 & -21.4 & -13.4 & -13.6 & -57.7 \\
\hline 4 & -78.0 & -91.5 & -96.7 & -85.4 & -100.0 & -101.6 \\
\hline 5 & -30.7 & -45.3 & -26.4 & -33.6 & -13.5 & -32.2 \\
\hline 6 & -4.8 & -2.7 & -17.0 & -12.5 & -20.3 & -53.3 \\
\hline 7 & -87.8 & -92.7 & -89.1 & -82.9 & -96.0 & -102.9 \\
\hline 8 & -6.3 & -2.8 & -12.1 & -4.2 & -17.0 & -27.7 \\
\hline 9 & -92.3 & -90.9 & -107.9 & -108.0 & -119.7 & -118.6 \\
\hline 10 & -241.3 & -266.8 & -242.2 & -255.0 & -267.0 & -248.9 \\
\hline 11 & -2.2 & -2.8 & -4.2 & -10.8 & -7.5 & -40.6 \\
\hline 12 & -83.0 & -82.7 & -70.7 & -101.0 & -101.9 & -83.5 \\
\hline 13 & 15.9 & -17.5 & -12.8 & -24.2 & -31.1 & -15.1 \\
\hline 14 & 8.6 & -30.5 & -39.1 & -24.6 & -19.4 & -57.5 \\
\hline 15 & -101.4 & -98.9 & -100.2 & -89.8 & -109.6 & -109.2 \\
\hline 16 & -92.0 & -102.9 & -90.4 & -75.8 & -98.9 & -100.8 \\
\hline 17 & 32.4 & 52.1 & 53.6 & 31.1 & 46.8 & -2.0 \\
\hline 18 & -17.5 & -18.7 & -28.5 & -22.4 & -45.8 & -62.2 \\
\hline 19 & -119.4 & -122.3 & -104.3 & -108.2 & -129.3 & -138.4 \\
\hline 20 & -24.9 & -26.1 & -34.9 & -42.3 & -22.1 & -61.2 \\
\hline 21 & -98.8 & -114.6 & -92.5 & -109.1 & -111.3 & -119.3 \\
\hline 22 & -5.3 & -5.0 & -4.4 & -1.1 & -24.2 & -45.4 \\
\hline 23 & -76.3 & -90.2 & -101.6 & -104.9 & -121.6 & -112.4 \\
\hline 24 & -23.6 & 10.5 & -22.7 & -22.9 & -36.4 & -57.9 \\
\hline
\end{tabular}


Binding Free Energies on M-MOF-808 Nodes
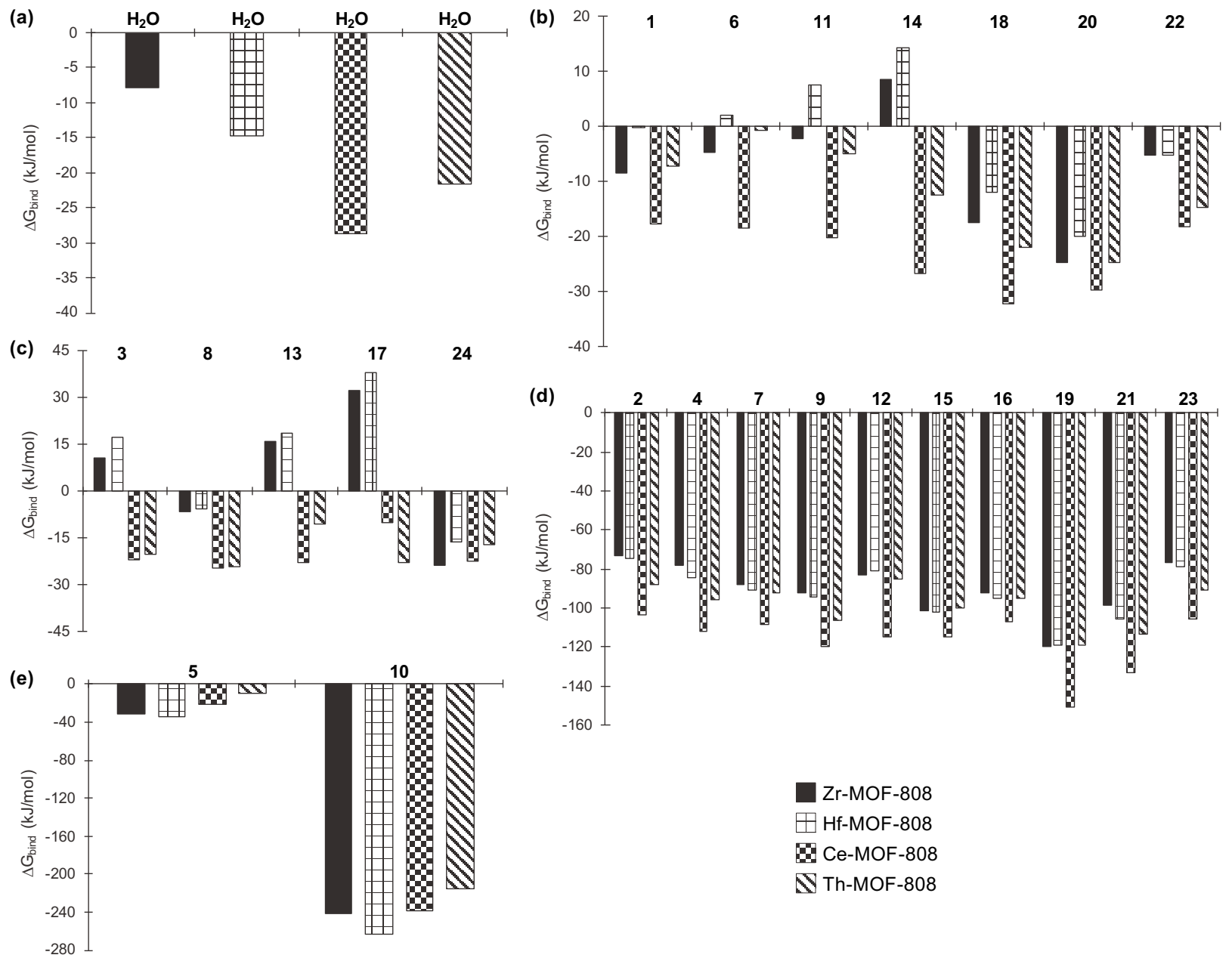

Figure S8. Binding free energies for (a) water, (b) nerve agent and simulant molecules, (c) alcohol and thiol hydrolysis product molecules, (d) bidentate anion products, and (e) monodentate anion products bound to the nodes of M-MOF-808 (M = Zr, Hf, Ce, Th). 


\section{Effect of Metal Electronegativity on Water Binding Energy: M(IV)-MOF Nodes}

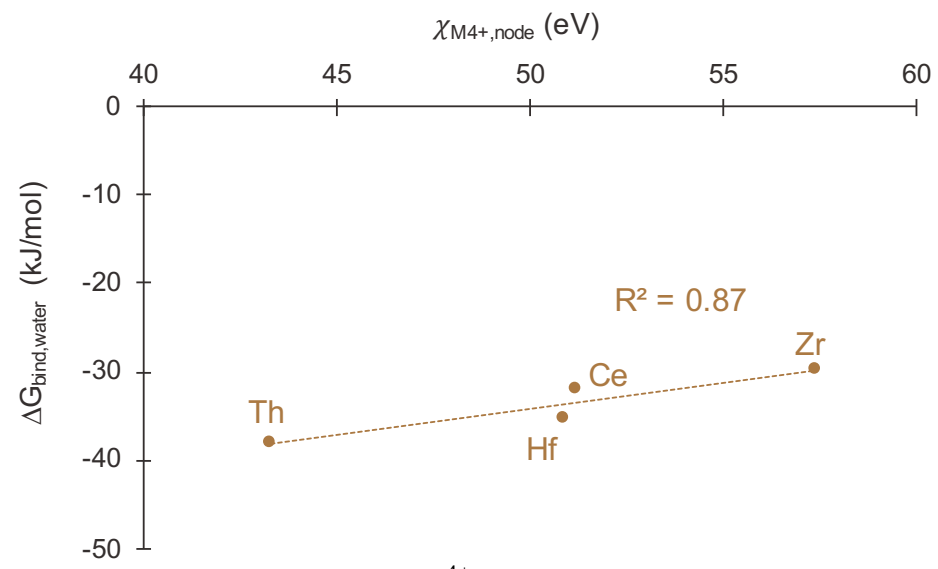

Figure S9. The effect of the electronegativity of $\mathrm{M}^{4+}$ cations in the nodes of M-bi(trans)-defect UiO-66 on the binding free energy for water.

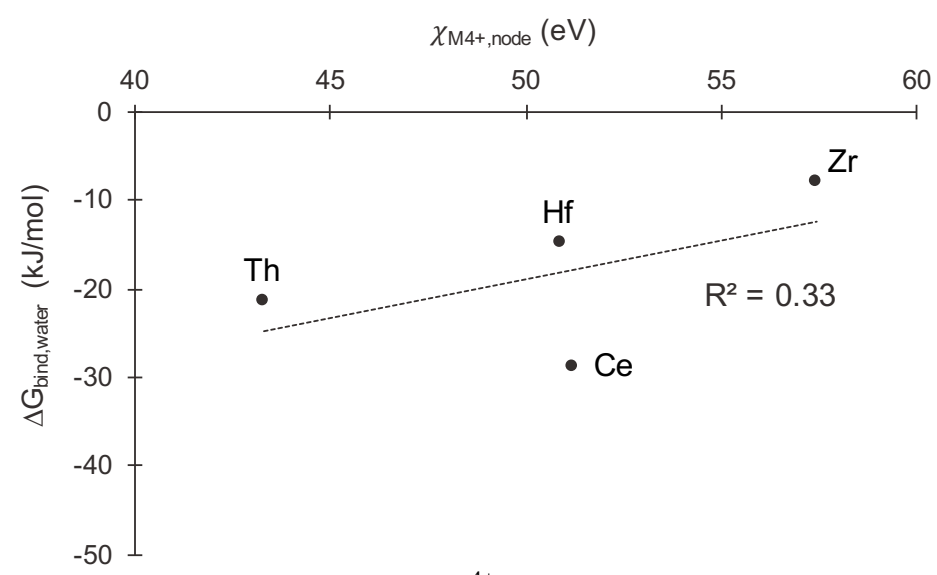

Figure S10. The effect of the electronegativity of $\mathrm{M}^{4+}$ cations in the nodes of M-MOF- 808 on the binding free energy for water. 


\section{Binding Free Energy Values for All Molecules: M(IV)-MOF Nodes}

Table S4. Binding free energies (in $\mathrm{kJ} / \mathrm{mol}$ ) for molecules bound to M-bi(trans)-defect UiO-66 node sites, corresponding to results in Figure 3. Negative and positive values indicate favorable and unfavorable binding, respectively.

\begin{tabular}{|c|c|c|c|c|}
\hline Molecule & $\mathrm{Zr}$ & Hf & $\mathrm{Ce}$ & Th \\
\hline $\mathrm{H}_{2} \mathrm{O}$ & -29.9 & -35.4 & -32.0 & -38.2 \\
\hline 1 & 4.6 & -4.6 & -27.2 & -19.0 \\
\hline 2 & -100.4 & -103.6 & -135.3 & -86.8 \\
\hline 3 & -21.4 & -29.5 & -30.6 & -37.6 \\
\hline 4 & -96.7 & -99.7 & -129.9 & -89.0 \\
\hline 5 & -26.4 & -26.6 & -27.8 & -21.1 \\
\hline 6 & -17.0 & -25.5 & -31.1 & -12.7 \\
\hline 7 & -89.1 & -92.4 & -131.4 & -71.0 \\
\hline 8 & -12.1 & -17.6 & -27.5 & -34.5 \\
\hline 9 & -107.9 & -110.6 & -135.5 & -92.5 \\
\hline 10 & -242.2 & -242.8 & -250.1 & -229.1 \\
\hline 11 & -4.2 & -13.2 & -30.2 & -19.3 \\
\hline 12 & -70.7 & -75.0 & -127.4 & -58.9 \\
\hline 13 & -12.8 & -33.5 & -30.3 & -36.5 \\
\hline 14 & -39.1 & -40.7 & -59.9 & -45.9 \\
\hline 15 & -100.2 & -104.4 & -130.1 & -101.6 \\
\hline 16 & -90.4 & -96.5 & -145.0 & -101.5 \\
\hline 17 & 53.6 & 42.3 & 2.9 & 33.9 \\
\hline 18 & -28.5 & -33.5 & -42.4 & -28.9 \\
\hline 19 & -104.3 & -110.0 & -157.4 & -87.4 \\
\hline 20 & -34.9 & -41.1 & -48.0 & -32.5 \\
\hline 21 & -92.5 & -95.2 & -154.7 & -95.1 \\
\hline 22 & -4.4 & -10.5 & -26.5 & -17.4 \\
\hline 23 & -101.6 & -105.1 & -130.9 & -84.0 \\
\hline 24 & -22.7 & -29.3 & -28.9 & -60.2 \\
\hline
\end{tabular}


Table S5. Binding free energies (in $\mathrm{kJ} / \mathrm{mol}$ ) for molecules bound to M-MOF-808 node sites, corresponding to results in Figure S8. Negative and positive values indicate favorable and unfavorable binding, respectively.

\begin{tabular}{|c|c|c|c|c|}
\hline Molecule & $\mathbf{Z r}$ & $\mathbf{H f}$ & $\mathbf{C e}$ & $\mathbf{T h}$ \\
\hline $\mathbf{H}_{\mathbf{2}} \mathbf{O}$ & -7.9 & -14.8 & -28.8 & -21.6 \\
\hline $\mathbf{1}$ & -8.5 & -0.4 & -17.9 & -7.4 \\
\hline $\mathbf{2}$ & -72.9 & -74.6 & -103.4 & -88.0 \\
\hline $\mathbf{3}$ & 10.8 & 17.4 & -21.8 & -20.2 \\
\hline $\mathbf{4}$ & -78.0 & -84.1 & -112.1 & -95.5 \\
\hline $\mathbf{5}$ & -30.7 & -34.6 & -20.6 & -9.5 \\
\hline $\mathbf{6}$ & -4.8 & 1.9 & -18.5 & -0.7 \\
\hline $\mathbf{7}$ & -87.8 & -90.8 & -108.3 & -92.5 \\
\hline $\mathbf{8}$ & -6.3 & -5.8 & -24.6 & -24.1 \\
\hline $\mathbf{9}$ & -92.3 & -94.4 & -120.0 & -106.0 \\
\hline $\mathbf{1 0}$ & -241.3 & -263.1 & -239.2 & -214.9 \\
\hline $\mathbf{1 1}$ & -2.2 & 7.4 & -20.2 & -5.0 \\
\hline $\mathbf{1 2}$ & -83.0 & -80.8 & -115.0 & -85.3 \\
\hline $\mathbf{1 3}$ & 15.9 & 18.7 & -23.0 & -10.6 \\
\hline $\mathbf{1 4}$ & 8.6 & 14.2 & -26.8 & -12.5 \\
\hline $\mathbf{1 5}$ & -101.4 & -102.1 & -114.8 & -100.0 \\
\hline $\mathbf{1 6}$ & -92.0 & -95.0 & -107.2 & -95.3 \\
\hline $\mathbf{1 7}$ & 32.4 & 37.9 & -10.3 & -22.7 \\
\hline $\mathbf{1 8}$ & -17.5 & -12.0 & -32.3 & -22.0 \\
\hline $\mathbf{1 9}$ & -119.4 & -118.9 & -150.4 & -119.2 \\
\hline $\mathbf{2 0}$ & -24.9 & -20.0 & -29.8 & -24.8 \\
\hline $\mathbf{2 1}$ & -98.8 & -105.3 & -133.1 & -113.5 \\
\hline $\mathbf{2 2}$ & -5.3 & -5.2 & -18.3 & -14.8 \\
\hline $\mathbf{2 3}$ & -76.3 & -79.0 & -105.9 & -90.9 \\
\hline $\mathbf{2 4}$ & -23.6 & -16.4 & -22.2 & -17.0 \\
\hline & & & & \\
\hline
\end{tabular}




\section{QSAR Modeling: Molecular Descriptors}

Table S6. List of molecular descriptors used in the QSAR models for the prediction of binding free energies.

\begin{tabular}{|c|c|c|c|}
\hline Descriptor & Notation & Unit & Range $^{a}$ \\
\hline NBO partial atomic charge on binding $\mathrm{O} / \mathrm{S}$ atom $^{b}$ & qo/S,mol & a.u. & $(-1.149)-(-0.148)$ \\
\hline average NBO partial atomic charge on binding $\mathrm{O}$ atoms ${ }^{c}$ & $A v q O, m o l$ & a.u. & $(-1.226)-(-1.189)$ \\
\hline Wiberg bond index of binding $\mathrm{O} / \mathrm{S}$ atom $^{b}$ & BIo/S,mol & & $1.424-2.041$ \\
\hline average Wiberg bond index of binding $\mathrm{O}$ atoms ${ }^{c}$ & $A v B I_{O, m o l}$ & & $1.308-1.368$ \\
\hline molecular volume & $V_{m o l}$ & $\mathrm{~cm}^{3} / \mathrm{mol}$ & $17.289-205.526,63.697-184.317$ \\
\hline solvent-accessible surface area & $S A_{m o l}$ & $\AA^{2}$ & $113.827-483.313,220.756-420$ \\
\hline molecular dipole moment & $\mu_{m o l}$ & Debye & $1.704-7.723,4.911-16.278$ \\
\hline total number of atoms in molecule & $n A t_{m o l}$ & & $3-42,9-35$ \\
\hline number of electrons in molecule & $n$ Elec $_{\text {mol }}$ & & $10-146,50-130$ \\
\hline molecular ionization potential & $I P_{m o l}$ & hartree & $0.261-0.383,0.254-0.318$ \\
\hline molecular electron affinity & $E A_{m o l}$ & hartree & $(-0.112)-(-0.019),(-0.115)-(-0.057)$ \\
\hline molecular hardness & $\eta_{m o l}$ & hartree & $0.160-0.246,0.162-0.217$ \\
\hline molecular softness & $S_{m o l}$ & hartree $^{-1}$ & $4.057-6.259,4.611-6.180$ \\
\hline molecular electronegativity & $\chi_{m o l}$ & hartree & $0.092-0.147,0.081-0.108$ \\
\hline molecular electrophilicity & $\omega_{m o l}$ & hartree & $0.025-0.065,0.018-0.034$ \\
\hline number of hydrogen atoms in molecule & $n H_{m o l}$ & & $2-26,4-21$ \\
\hline number of carbon atoms in molecule & $n C_{m o l}$ & & $0-11,1-9$ \\
\hline number of nitrogen atoms in molecule & $n N_{m o l}$ & & $0-2,0-2$ \\
\hline number of oxygen atoms in molecule ${ }^{b}$ & $n O_{m o l}$ & & $0-3$ \\
\hline number of non-hydrogen atoms in molecule & $n N o n H_{m o l}$ & & $1-16,5-14$ \\
\hline number of rotatable bonds in molecule & $n R B_{m o l}$ & & $0-8,0-6$ \\
\hline number of donor atoms for $\mathrm{H}$-bonds in molecule ${ }^{b}$ & $n H B d_{m o l}$ & & $0-2$ \\
\hline number of acceptor atoms for $\mathrm{H}$-bonds in molecule & $n H B a_{m o l}$ & & $1-5,3-5$ \\
\hline molecular unsaturation index ${ }^{b}$ & $U I_{m o l}$ & & $0-1.585$ \\
\hline molecular hydrophilicity index & $H y I_{m o l}$ & & $(-0.673)-6.169,(-0.626)-0.671$ \\
\hline molecular weight & $M W_{m o l}$ & $\mathrm{~g} / \mathrm{mol}$ & $18.015-267.368,95.014-238.306$ \\
\hline average molecular weight & $A M W_{\text {mol }}$ & $\mathrm{g} / \mathrm{mol}$ & $4.866-7.783,6.636-10.557$ \\
\hline
\end{tabular}

${ }^{a}$ The values in red and green correspond to neutral molecules and bidentate anions, respectively. ${ }^{b}$ These descriptors were only computed for neutral molecules. ${ }^{c}$ These descriptors were only computed for bidentate anions. 
Below, we provide more explicit definitions and equations for some of the molecular descriptors described in the main text and shown in Table S6.

Molecular ionization potential $\left(I P_{m o l}\right)$ : refers to the amount of energy required to remove the valence electron from an isolated neutral species. Koopmans' theorem ${ }^{15}$ may be used to relate the ionization potential to the energy of the highest occupied molecular orbital, where $I P_{\text {mоl }} \approx-E_{\text {Номо. }}$

Molecular electron affinity $\left(E A_{m o l}\right)$ : refers to the difference in energy of a neutral species and its anion. Koopmans' theorem ${ }^{15}$ may be used to relate the electron affinity to the energy of the lowest unoccupied molecular orbital, where $E A_{m o l} \approx-E_{L U M O}$.

Molecular hardness $\left(\eta_{\text {mol }}\right)$ : measures the stability of a molecule.

Molecular softness $\left(S_{m o l}\right)$ : measures the reactivity of a molecule.

Molecular electronegativity $\left(\chi_{m o l}\right)$ : describes the strength with which a species attracts electrons.

Molecular electrophilicity $\left(\omega_{\text {mol }}\right)$ : measures the reactivity of a species towards attracting electrons from a nucleophile.

Using Koopmans' theorem, Parr and co-workers ${ }^{16}$ derived $\eta_{m o l}, S_{m o l}, \chi_{m o l}$, and $\omega_{m o l}$ as:

$$
\begin{aligned}
& \eta_{m o l}=\frac{I P_{m o l}-E A_{m o l}}{2} \\
& S_{m o l}=\frac{1}{\eta_{m o l}} \\
& \chi_{m o l}=\frac{I P_{m o l}+E A_{m o l}}{2} \\
& \omega_{m o l}=\frac{\chi_{m o l}{ }^{2}}{2 \eta_{m o l}}
\end{aligned}
$$

Molecular unsaturation index $\left(U I_{m o l}\right)$ : measures the extent of unsaturated bonds in a molecule. The definition as described in the user manual for Dragon ${ }^{17}$ is:

$$
U I_{m o l}=\log _{2}\left(1+n D B_{m o l}+n T B_{m o l}+n A B_{m o l}\right)
$$

where $n D B_{m o l}, n T B_{m o l}$, and $n A B_{m o l}$ denote the number of double, triple, and aromatic bonds in the molecule, respectively.

Molecular hydrophilicity index $\left(H y I_{m o l}\right):$ measures the extent of hydrophilicity of a molecule. ${ }^{18}$

$$
H y I_{m o l}=\frac{\left(1+n H y_{m o l}\right) \log _{2}\left(1+n H y_{m o l}\right)+n C_{m o l}\left(\frac{1}{n N o n H_{m o l}} \log _{2} \frac{1}{n N o n H_{m o l}}\right)+\sqrt{\frac{n H y_{m o l}}{\left(n N o n H_{m o l}\right)^{2}}}}{\log _{2}\left(1+n N o n H_{m o l}\right)}
$$


where $n H y_{m o l}$ denotes the number of hydrophilic groups (number of $\mathrm{H}$ atoms bonded to $\mathrm{O}, \mathrm{S}$, or $\mathrm{N}$ atoms) in the molecule.

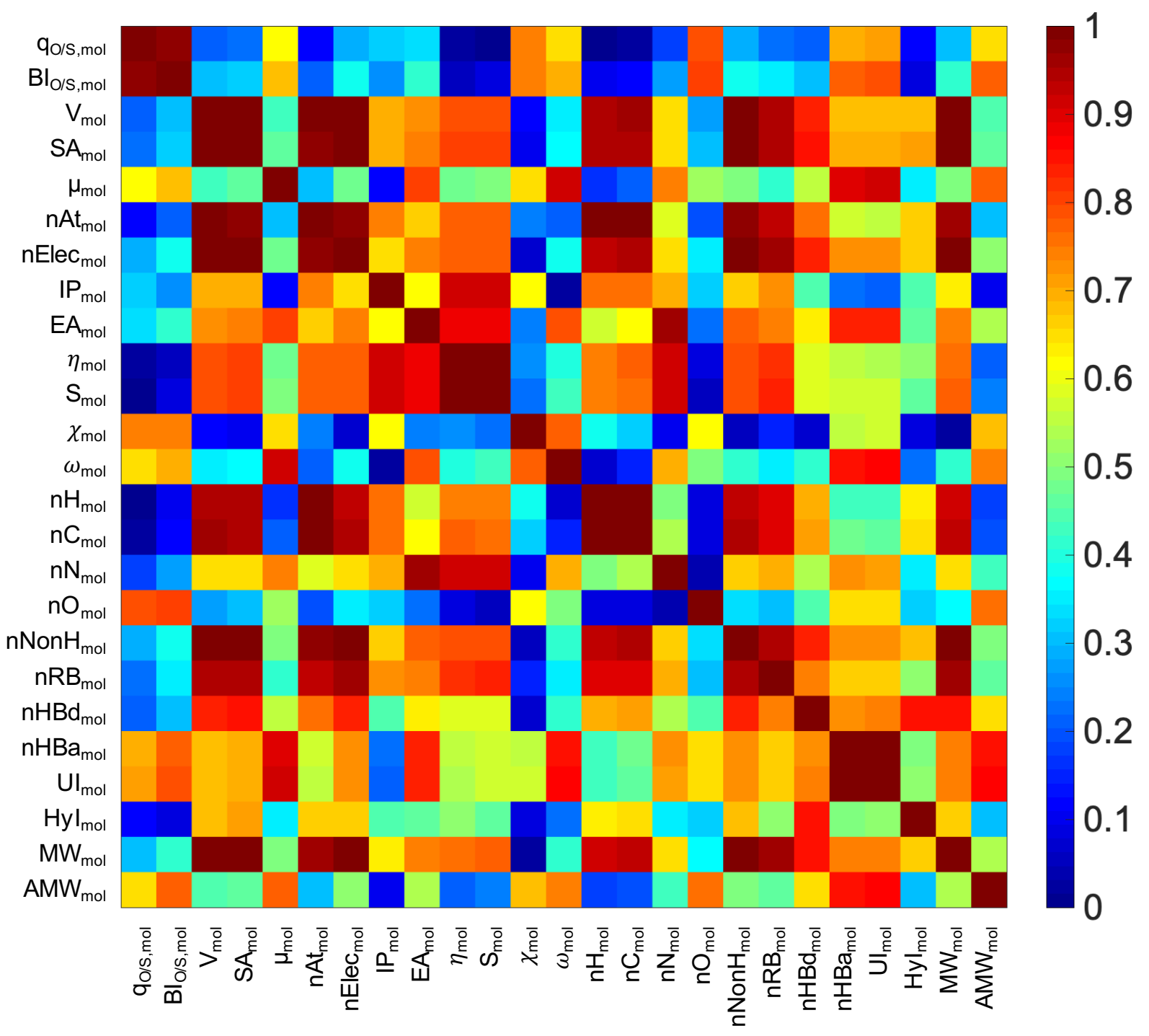

Figure S11. Heatmap of absolute value Pearson's correlation coefficients between molecular descriptors for the neutral molecules. 


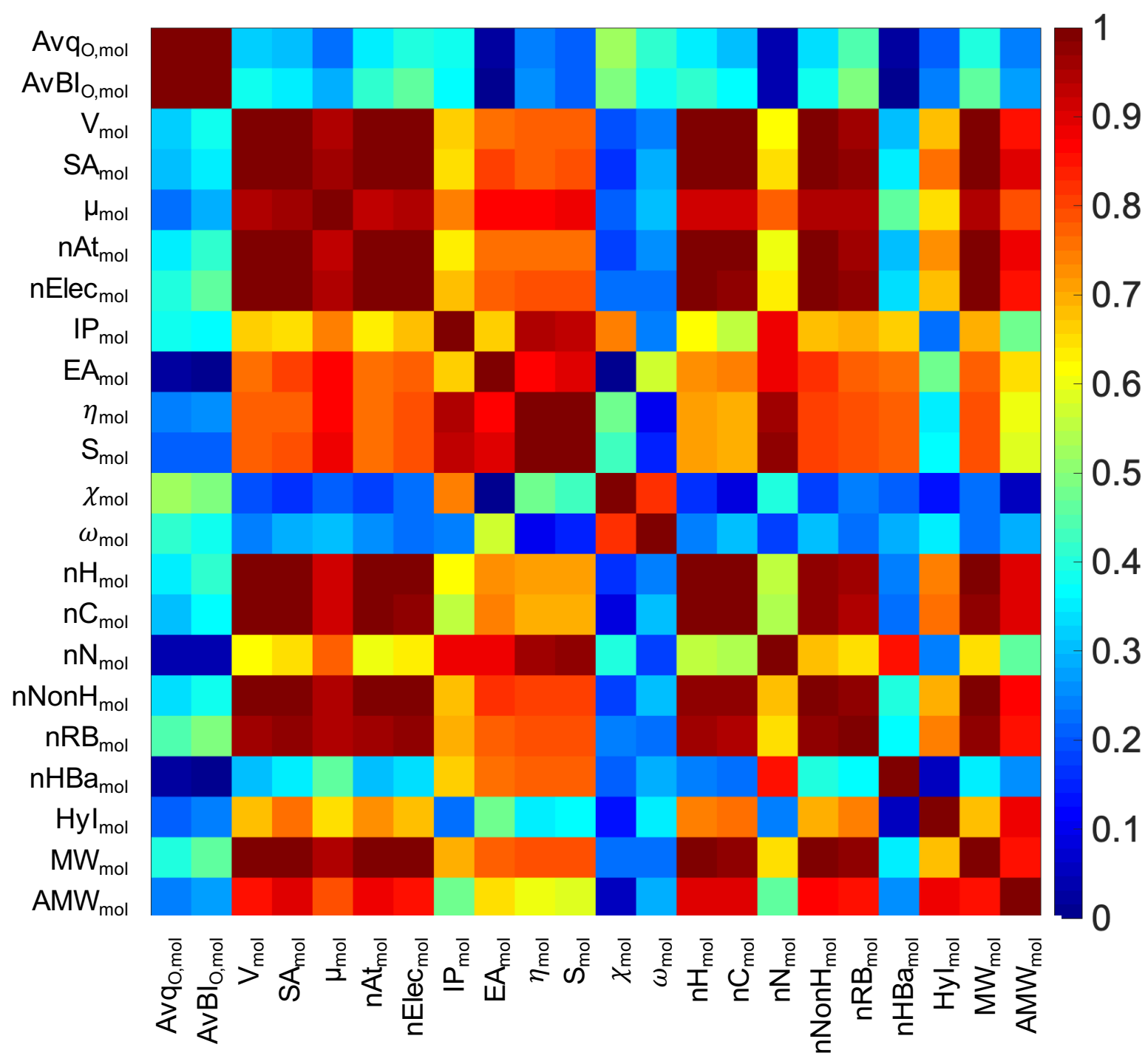

Figure S12. Heatmap of absolute value Pearson's correlation coefficients between molecular descriptors for the bidentate anions. 
Table S7. The unscaled values of the molecular descriptors for the neutral molecules used for QSAR modeling. For descriptor units, refer to Table S6.

\begin{tabular}{|c|c|c|c|c|c|c|c|c|c|c|}
\hline Molecule & qo/S,mol & BIo/S,mol & $V_{m o l}$ & $S A_{m o l}$ & $\mu_{m o l}$ & $n A t_{m o l}$ & nElec $_{\text {mol }}$ & $I P_{m o l}$ & $E A_{m o l}$ & $\eta_{m o l}$ \\
\hline $\mathrm{H}_{2} \mathrm{O}$ & -0.972 & 1.533 & 17.289 & 113.827 & 2.440 & 3 & 10 & 0.383 & -0.110 & 0.246 \\
\hline 1 & -1.098 & 1.492 & 125.453 & 342.293 & 7.046 & 21 & 86 & 0.313 & -0.019 & 0.166 \\
\hline 3 & -0.808 & 1.735 & 40.278 & 184.254 & 2.104 & 9 & 26 & 0.338 & -0.107 & 0.222 \\
\hline 6 & -1.127 & 1.459 & 102.993 & 291.424 & 3.721 & 18 & 74 & 0.372 & -0.094 & 0.233 \\
\hline 8 & -0.814 & 1.736 & 63.600 & 209.187 & 2.184 & 12 & 34 & 0.338 & -0.100 & 0.219 \\
\hline 11 & -1.125 & 1.460 & 145.404 & 347.746 & 3.749 & 27 & 98 & 0.366 & -0.086 & 0.226 \\
\hline 13 & -0.809 & 1.745 & 89.137 & 270.280 & 2.269 & 21 & 58 & 0.330 & -0.094 & 0.212 \\
\hline 14 & -1.127 & 1.460 & 205.526 & 483.313 & 2.713 & 42 & 146 & 0.261 & -0.058 & 0.160 \\
\hline 17 & -0.148 & 2.041 & 144.837 & 353.589 & 1.704 & 29 & 90 & 0.262 & -0.079 & 0.170 \\
\hline 18 & -1.149 & 1.424 & 152.558 & 377.772 & 7.723 & 28 & 104 & 0.292 & -0.030 & 0.161 \\
\hline 20 & -1.137 & 1.445 & 155.593 & 388.342 & 6.867 & 29 & 112 & 0.298 & -0.027 & 0.162 \\
\hline 22 & -1.136 & 1.441 & 91.313 & 277.482 & 5.697 & 16 & 66 & 0.370 & -0.096 & 0.233 \\
\hline 24 & -0.793 & 1.745 & 28.765 & 146.818 & 2.123 & 6 & 18 & 0.343 & -0.112 & 0.227 \\
\hline
\end{tabular}

\begin{tabular}{|c|c|c|c|c|c|c|c|c|c|c|}
\hline Molecule & $\boldsymbol{S}_{\boldsymbol{m o l}}$ & $\boldsymbol{\chi}_{\boldsymbol{m o l}}$ & $\boldsymbol{\omega}_{\boldsymbol{m o l}}$ & $\boldsymbol{n} \boldsymbol{H}_{\boldsymbol{m o l}}$ & $\boldsymbol{n} \boldsymbol{C}_{\boldsymbol{m o l}}$ & $\boldsymbol{n N}_{\boldsymbol{m o l}}$ & $\boldsymbol{n O _ { m o l }}$ & $\boldsymbol{n N o n H _ { m o l }}$ & $\boldsymbol{n R B _ { m o l }}$ & $\boldsymbol{n H B d _ { m o l }}$ \\
\hline $\mathbf{H}_{\mathbf{2}} \mathbf{O}$ & 4.057 & 0.136 & 0.038 & 2 & 0 & 0 & 1 & 1 & 0 & 2 \\
\hline $\mathbf{1}$ & 6.031 & 0.147 & 0.065 & 11 & 5 & 2 & 2 & 10 & 4 & 0 \\
\hline $\mathbf{3}$ & 4.498 & 0.115 & 0.030 & 6 & 2 & 0 & 1 & 3 & 0 & 1 \\
\hline $\mathbf{6}$ & 4.291 & 0.139 & 0.041 & 10 & 4 & 0 & 2 & 8 & 2 & 0 \\
\hline $\mathbf{8}$ & 4.566 & 0.119 & 0.033 & 8 & 3 & 0 & 1 & 4 & 0 & 1 \\
\hline $\mathbf{1 1}$ & 4.429 & 0.140 & 0.043 & 16 & 7 & 0 & 2 & 11 & 3 & 0 \\
\hline $\mathbf{1 3}$ & 4.723 & 0.118 & 0.033 & 14 & 6 & 0 & 1 & 7 & 1 & 1 \\
\hline $\mathbf{1 4}$ & 6.259 & 0.101 & 0.032 & 26 & 11 & 1 & 2 & 16 & 8 & 0 \\
\hline $\mathbf{1 7}$ & 5.873 & 0.092 & 0.025 & 19 & 8 & 1 & 0 & 10 & 4 & 0 \\
\hline $\mathbf{1 8}$ & 6.208 & 0.131 & 0.053 & 16 & 7 & 2 & 1 & 12 & 4 & 0 \\
\hline $\mathbf{2 0}$ & 6.158 & 0.135 & 0.056 & 16 & 7 & 2 & 2 & 13 & 5 & 0 \\
\hline $\mathbf{2 2}$ & 4.286 & 0.137 & 0.040 & 9 & 3 & 0 & 3 & 7 & 2 & 0 \\
\hline $\mathbf{2 4}$ & 4.402 & 0.116 & 0.029 & 4 & 1 & 0 & 1 & 2 & 0 & 1 \\
\hline
\end{tabular}


Table S7 (continued).

\begin{tabular}{|c|c|c|c|c|c|}
\hline Molecule & $\boldsymbol{n H B a}_{\text {mol }}$ & $\boldsymbol{U I}_{\text {mol }}$ & $\boldsymbol{H y I}_{\text {mol }}$ & $\boldsymbol{M W}_{\text {mol }}$ & $\boldsymbol{A M}_{\text {mol }}$ \\
\hline $\mathbf{H}_{\mathbf{2}} \mathbf{O}$ & 1 & 0 & 6.169 & 18.015 & 6.005 \\
\hline $\mathbf{1}$ & 4 & 1.585 & -0.480 & 162.129 & 7.720 \\
\hline $\mathbf{3}$ & 1 & 0 & 0.638 & 46.069 & 5.119 \\
\hline $\mathbf{6}$ & 3 & 1 & -0.473 & 140.094 & 7.783 \\
\hline $\mathbf{8}$ & 1 & 0 & 0.323 & 60.096 & 5.008 \\
\hline $\mathbf{1 1}$ & 3 & 1 & -0.614 & 182.175 & 6.747 \\
\hline $\mathbf{1 3}$ & 1 & 0 & -0.088 & 102.177 & 4.866 \\
\hline $\mathbf{1 4}$ & 3 & 1 & -0.673 & 267.368 & 6.366 \\
\hline $\mathbf{1 7}$ & 1 & 0 & -0.161 & 161.307 & 5.562 \\
\hline $\mathbf{1 8}$ & 4 & 1.585 & -0.565 & 194.190 & 6.935 \\
\hline $\mathbf{2 0}$ & 5 & 1.585 & -0.523 & 210.189 & 7.248 \\
\hline $\mathbf{2 2}$ & 3 & 1 & -0.401 & 124.076 & 7.755 \\
\hline $\mathbf{2 4}$ & 1 & 0 & 1.262 & 32.042 & 5.340 \\
\hline
\end{tabular}

Table S8. The unscaled values of the molecular descriptors for the bidentate anions used for QSAR modeling. For descriptor units, refer to Table S6.

\begin{tabular}{|c|c|c|c|c|c|c|c|c|c|c|}
\hline Molecule & $\boldsymbol{A v q}_{\boldsymbol{O}, \boldsymbol{m o l}}$ & $\boldsymbol{A v B I}_{\boldsymbol{O}, \boldsymbol{m o l}}$ & $\boldsymbol{V}_{\boldsymbol{m o l}}$ & $\boldsymbol{S A}_{\boldsymbol{m o l}}$ & $\boldsymbol{\mu}_{\boldsymbol{m o l}}$ & $\boldsymbol{n A t}_{\boldsymbol{m o l}}$ & $\boldsymbol{n E l e c}_{\boldsymbol{m o l}}$ & $\boldsymbol{I P}_{\boldsymbol{m o l}}$ & $\boldsymbol{E A}_{\boldsymbol{m o l}}$ & $\boldsymbol{\eta}_{\boldsymbol{m o l}}$ \\
\hline $\mathbf{2}$ & -1.200 & 1.345 & 107.803 & 317.303 & 8.968 & 20 & 82 & 0.264 & -0.102 & 0.183 \\
\hline $\mathbf{4}$ & -1.204 & 1.339 & 78.980 & 253.376 & 7.104 & 14 & 66 & 0.271 & -0.105 & 0.188 \\
\hline $\mathbf{7}$ & -1.210 & 1.332 & 94.664 & 293.255 & 8.660 & 18 & 74 & 0.316 & -0.103 & 0.210 \\
\hline $\mathbf{9}$ & -1.217 & 1.321 & 63.697 & 220.756 & 4.911 & 9 & 50 & 0.318 & -0.115 & 0.217 \\
\hline $\mathbf{1 2}$ & -1.210 & 1.333 & 138.440 & 354.065 & 9.347 & 27 & 98 & 0.315 & -0.098 & 0.206 \\
\hline $\mathbf{1 5}$ & -1.210 & 1.332 & 89.112 & 279.764 & 7.724 & 15 & 66 & 0.318 & -0.113 & 0.215 \\
\hline $\mathbf{1 6}$ & -1.189 & 1.368 & 184.317 & 420.152 & 16.278 & 35 & 130 & 0.254 & -0.091 & 0.173 \\
\hline $\mathbf{1 9}$ & -1.226 & 1.308 & 152.276 & 386.074 & 15.554 & 28 & 104 & 0.258 & -0.070 & 0.164 \\
\hline $\mathbf{2 1}$ & -1.204 & 1.341 & 152.309 & 387.380 & 15.213 & 29 & 112 & 0.267 & -0.057 & 0.162 \\
\hline $\mathbf{2 3}$ & -1.209 & 1.332 & 66.722 & 249.963 & 6.559 & 12 & 58 & 0.318 & -0.114 & 0.216 \\
\hline
\end{tabular}


Table S8 (continued).

\begin{tabular}{|c|c|c|c|c|c|c|c|c|c|c|}
\hline Molecule & $\boldsymbol{S}_{\boldsymbol{m o l}}$ & $\boldsymbol{\chi}_{\boldsymbol{m o l}}$ & $\boldsymbol{\omega}_{\boldsymbol{m o l}}$ & $\boldsymbol{n H}_{\boldsymbol{m o l}}$ & $\boldsymbol{n \boldsymbol { C } _ { \boldsymbol { m o l } }}$ & $\boldsymbol{n N}_{\boldsymbol{m o l}}$ & $\boldsymbol{n N o n H _ { m o l }}$ & $\boldsymbol{n R B _ { m o l }}$ & $\boldsymbol{n H B a _ { m o l }}$ & $\boldsymbol{H y I}_{\boldsymbol{m o l}}$ \\
\hline $\mathbf{2}$ & 5.470 & 0.081 & 0.018 & 11 & 4 & 1 & 9 & 3 & 4 & -0.424 \\
\hline $\mathbf{4}$ & 5.324 & 0.083 & 0.018 & 7 & 2 & 1 & 7 & 1 & 4 & 0.447 \\
\hline $\mathbf{7}$ & 4.772 & 0.106 & 0.027 & 10 & 4 & 0 & 8 & 2 & 3 & -0.473 \\
\hline $\mathbf{9}$ & 4.611 & 0.101 & 0.024 & 4 & 1 & 0 & 5 & 0 & 3 & 0.671 \\
\hline $\mathbf{1 2}$ & 4.847 & 0.108 & 0.029 & 16 & 7 & 0 & 11 & 3 & 3 & -0.614 \\
\hline $\mathbf{1 5}$ & 4.643 & 0.103 & 0.024 & 8 & 3 & 0 & 7 & 2 & 3 & -0.401 \\
\hline $\mathbf{1 6}$ & 5.792 & 0.082 & 0.019 & 21 & 9 & 1 & 14 & 6 & 3 & -0.626 \\
\hline $\mathbf{1 9}$ & 6.082 & 0.094 & 0.027 & 16 & 7 & 2 & 12 & 4 & 4 & -0.565 \\
\hline $\mathbf{2 1}$ & 6.180 & 0.105 & 0.034 & 16 & 7 & 2 & 13 & 5 & 5 & -0.523 \\
\hline $\mathbf{2 3}$ & 4.632 & 0.102 & 0.024 & 6 & 2 & 0 & 6 & 1 & 3 & -0.307 \\
\hline
\end{tabular}

\begin{tabular}{|c|c|c|}
\hline Molecule & $\boldsymbol{M W}_{\text {mol }}$ & $\boldsymbol{A} \boldsymbol{M W}_{\text {mol }}$ \\
\hline $\mathbf{2}$ & 152.110 & 7.606 \\
\hline $\mathbf{4}$ & 124.056 & 8.861 \\
\hline $\mathbf{7}$ & 137.095 & 7.616 \\
\hline $\mathbf{9}$ & 95.014 & 10.557 \\
\hline $\mathbf{1 2}$ & 179.176 & 6.636 \\
\hline $\mathbf{1 5}$ & 123.068 & 8.205 \\
\hline $\mathbf{1 6}$ & 238.306 & 6.809 \\
\hline $\mathbf{1 9}$ & 191.191 & 6.828 \\
\hline $\mathbf{2 1}$ & 207.190 & 7.144 \\
\hline $\mathbf{2 3}$ & 109.041 & 9.087 \\
\hline
\end{tabular}

The formula used to scale descriptors was as follows:

$$
x_{i j}^{S}=\frac{x_{i j}-x_{j, \min }}{x_{j, \max }-x_{j, \min }}
$$

where $x_{i j}$ and $x_{i j}^{S}$ are the unscaled and scaled $j^{\text {th }}$ descriptor values for molecule $i$, respectively, and $x_{j, \min }$ and $x_{j, \max }$ are the minimum and maximum values for the $j^{\text {th }}$ descriptor. For all descriptors, $\min \left(x_{i j}^{s}\right)=0$ and $\max \left(x_{i j}^{s}\right)=1$. Scaling was performed separately for the neutral molecules descriptors and the bidentate anions descriptors. 
Table S9. The scaled values of the molecular descriptors for the neutral molecules used for QSAR modeling.

\begin{tabular}{|c|c|c|c|c|c|c|c|c|c|c|}
\hline Molecule & qo/S,mol & BIo/S,mol & $V_{m o l}$ & $S A_{m o l}$ & $\mu_{m o l}$ & $n A t_{m o l}$ & nElec $_{\text {mol }}$ & $I P_{m o l}$ & $E A_{m o l}$ & $\eta_{m o l}$ \\
\hline $\mathrm{H}_{2} \mathrm{O}$ & 0.177 & 0.176 & 0.000 & 0.000 & 0.122 & 0.000 & 0.000 & 1.000 & 0.013 & 1.000 \\
\hline 1 & 0.051 & 0.110 & 0.575 & 0.618 & 0.887 & 0.462 & 0.559 & 0.423 & 1.000 & 0.070 \\
\hline 3 & 0.341 & 0.504 & 0.122 & 0.191 & 0.066 & 0.154 & 0.118 & 0.631 & 0.050 & 0.722 \\
\hline 6 & 0.023 & 0.057 & 0.455 & 0.481 & 0.335 & 0.385 & 0.471 & 0.912 & 0.189 & 0.845 \\
\hline 8 & 0.335 & 0.505 & 0.246 & 0.258 & 0.080 & 0.231 & 0.176 & 0.635 & 0.128 & 0.683 \\
\hline 11 & 0.024 & 0.060 & 0.681 & 0.633 & 0.340 & 0.615 & 0.647 & 0.862 & 0.279 & 0.761 \\
\hline 13 & 0.340 & 0.521 & 0.382 & 0.423 & 0.094 & 0.462 & 0.353 & 0.563 & 0.191 & 0.599 \\
\hline 14 & 0.022 & 0.060 & 1.000 & 1.000 & 0.168 & 1.000 & 1.000 & 0.000 & 0.575 & 0.000 \\
\hline 17 & 1.000 & 1.000 & 0.678 & 0.649 & 0.000 & 0.667 & 0.588 & 0.005 & 0.355 & 0.121 \\
\hline 18 & 0.000 & 0.000 & 0.719 & 0.714 & 1.000 & 0.641 & 0.691 & 0.254 & 0.880 & 0.015 \\
\hline 20 & 0.012 & 0.034 & 0.735 & 0.743 & 0.858 & 0.667 & 0.750 & 0.301 & 0.914 & 0.030 \\
\hline 22 & 0.013 & 0.028 & 0.393 & 0.443 & 0.663 & 0.333 & 0.412 & 0.898 & 0.164 & 0.848 \\
\hline 24 & 0.355 & 0.520 & 0.061 & 0.089 & 0.070 & 0.077 & 0.059 & 0.672 & 0.000 & 0.778 \\
\hline
\end{tabular}

\begin{tabular}{|c|c|c|c|c|c|c|c|c|c|c|}
\hline Molecule & $\boldsymbol{S}_{\boldsymbol{m o l}}$ & $\boldsymbol{\chi}_{\text {mol }}$ & $\boldsymbol{\omega}_{\text {mol }}$ & $\boldsymbol{n} \boldsymbol{H}_{\text {mol }}$ & $\boldsymbol{n} \boldsymbol{C}_{\text {mol }}$ & $\boldsymbol{n N}_{\boldsymbol{m o l}}$ & $\boldsymbol{n \boldsymbol { O } _ { \text { mol } }}$ & $\boldsymbol{n N o n H _ { \text { mol } }}$ & $\boldsymbol{n R B _ { \text { mol } }}$ & $\boldsymbol{n H B d _ { \text { mol } }}$ \\
\hline $\mathbf{H}_{\mathbf{2}} \mathbf{O}$ & 0.000 & 0.807 & 0.322 & 0.000 & 0.000 & 0.000 & 0.333 & 0.000 & 0.000 & 1.000 \\
\hline $\mathbf{1}$ & 0.896 & 1.000 & 1.000 & 0.375 & 0.455 & 1.000 & 0.667 & 0.600 & 0.500 & 0.000 \\
\hline $\mathbf{3}$ & 0.200 & 0.433 & 0.133 & 0.167 & 0.182 & 0.000 & 0.333 & 0.133 & 0.000 & 0.500 \\
\hline $\mathbf{6}$ & 0.106 & 0.858 & 0.416 & 0.333 & 0.364 & 0.000 & 0.667 & 0.467 & 0.250 & 0.000 \\
\hline $\mathbf{8}$ & 0.231 & 0.503 & 0.195 & 0.250 & 0.273 & 0.000 & 0.333 & 0.200 & 0.000 & 0.500 \\
\hline $\mathbf{1 1}$ & 0.169 & 0.879 & 0.467 & 0.583 & 0.636 & 0.000 & 0.667 & 0.667 & 0.375 & 0.000 \\
\hline $\mathbf{1 3}$ & 0.302 & 0.476 & 0.203 & 0.500 & 0.545 & 0.000 & 0.333 & 0.400 & 0.125 & 0.500 \\
\hline $\mathbf{1 4}$ & 1.000 & 0.180 & 0.189 & 1.000 & 1.000 & 0.500 & 0.667 & 1.000 & 1.000 & 0.000 \\
\hline $\mathbf{1 7}$ & 0.825 & 0.000 & 0.000 & 0.708 & 0.727 & 0.500 & 0.000 & 0.600 & 0.500 & 0.000 \\
\hline $\mathbf{1 8}$ & 0.977 & 0.714 & 0.709 & 0.583 & 0.636 & 1.000 & 0.333 & 0.733 & 0.500 & 0.000 \\
\hline $\mathbf{2 0}$ & 0.954 & 0.794 & 0.788 & 0.583 & 0.636 & 1.000 & 0.667 & 0.800 & 0.625 & 0.000 \\
\hline $\mathbf{2 2}$ & 0.104 & 0.821 & 0.385 & 0.292 & 0.273 & 0.000 & 1.000 & 0.400 & 0.250 & 0.000 \\
\hline $\mathbf{2 4}$ & 0.156 & 0.436 & 0.119 & 0.083 & 0.091 & 0.000 & 0.333 & 0.067 & 0.000 & 0.500 \\
\hline
\end{tabular}


Table S9 (continued).

\begin{tabular}{|c|c|c|c|c|c|}
\hline Molecule & $\boldsymbol{n H B a}_{\text {mol }}$ & $\boldsymbol{U I}_{\text {mol }}$ & $\boldsymbol{H y I}_{\text {mol }}$ & $\boldsymbol{M W}_{\text {mol }}$ & $\boldsymbol{A M}_{\text {mol }}$ \\
\hline $\mathbf{H}_{\mathbf{2}} \mathbf{O}$ & 0.000 & 0.000 & 1.000 & 0.000 & 0.391 \\
\hline $\mathbf{1}$ & 0.750 & 1.000 & 0.028 & 0.578 & 0.979 \\
\hline $\mathbf{3}$ & 0.000 & 0.000 & 0.192 & 0.113 & 0.087 \\
\hline $\mathbf{6}$ & 0.500 & 0.631 & 0.029 & 0.490 & 1.000 \\
\hline $\mathbf{8}$ & 0.000 & 0.000 & 0.146 & 0.169 & 0.049 \\
\hline $\mathbf{1 1}$ & 0.500 & 0.631 & 0.009 & 0.658 & 0.645 \\
\hline $\mathbf{1 3}$ & 0.000 & 0.000 & 0.085 & 0.338 & 0.000 \\
\hline $\mathbf{1 4}$ & 0.500 & 0.631 & 0.000 & 1.000 & 0.514 \\
\hline $\mathbf{1 7}$ & 0.000 & 0.000 & 0.075 & 0.575 & 0.239 \\
\hline $\mathbf{1 8}$ & 0.750 & 1.000 & 0.016 & 0.707 & 0.709 \\
\hline $\mathbf{2 0}$ & 1.000 & 1.000 & 0.022 & 0.771 & 0.817 \\
\hline $\mathbf{2 2}$ & 0.500 & 0.631 & 0.040 & 0.425 & 0.990 \\
\hline $\mathbf{2 4}$ & 0.000 & 0.000 & 0.283 & 0.056 & 0.163 \\
\hline
\end{tabular}

Table S10. The scaled values of the molecular descriptors for the bidentate anions used for QSAR modeling.

\begin{tabular}{|c|c|c|c|c|c|c|c|c|c|c|}
\hline Molecule & $\boldsymbol{A v q}_{\boldsymbol{o}, \boldsymbol{m o l}}$ & $\boldsymbol{A v B I}_{\boldsymbol{o} \text {,mol }}$ & $\boldsymbol{V}_{\boldsymbol{m o l}}$ & $\boldsymbol{S A}_{\text {mol }}$ & $\boldsymbol{\mu}_{\text {mol }}$ & $\boldsymbol{n A t}_{\text {mol }}$ & $\boldsymbol{n E l e c}_{\boldsymbol{m o l}}$ & $\boldsymbol{I P}_{\text {mol }}$ & $\boldsymbol{E A}_{\text {mol }}$ & $\boldsymbol{\eta}_{\text {mol }}$ \\
\hline $\mathbf{2}$ & 0.702 & 0.618 & 0.366 & 0.484 & 0.357 & 0.423 & 0.400 & 0.143 & 0.227 & 0.381 \\
\hline $\mathbf{4}$ & 0.577 & 0.513 & 0.127 & 0.164 & 0.193 & 0.192 & 0.200 & 0.252 & 0.176 & 0.472 \\
\hline $\mathbf{7}$ & 0.418 & 0.392 & 0.257 & 0.364 & 0.330 & 0.346 & 0.300 & 0.959 & 0.204 & 0.867 \\
\hline $\mathbf{9}$ & 0.228 & 0.209 & 0.000 & 0.000 & 0.000 & 0.000 & 0.000 & 1.000 & 0.000 & 1.000 \\
\hline $\mathbf{1 2}$ & 0.409 & 0.405 & 0.620 & 0.669 & 0.390 & 0.692 & 0.600 & 0.944 & 0.299 & 0.808 \\
\hline $\mathbf{1 5}$ & 0.433 & 0.397 & 0.211 & 0.296 & 0.247 & 0.231 & 0.200 & 0.995 & 0.046 & 0.973 \\
\hline $\mathbf{1 6}$ & 1.000 & 1.000 & 1.000 & 1.000 & 1.000 & 1.000 & 1.000 & 0.000 & 0.418 & 0.197 \\
\hline $\mathbf{1 9}$ & 0.000 & 0.000 & 0.734 & 0.829 & 0.936 & 0.731 & 0.675 & 0.061 & 0.765 & 0.047 \\
\hline $\mathbf{2 1}$ & 0.584 & 0.550 & 0.735 & 0.836 & 0.906 & 0.769 & 0.775 & 0.196 & 1.000 & 0.000 \\
\hline $\mathbf{2 3}$ & 0.441 & 0.404 & 0.025 & 0.146 & 0.145 & 0.115 & 0.100 & 0.993 & 0.026 & 0.982 \\
\hline
\end{tabular}


Table S10 (continued).

\begin{tabular}{|c|c|c|c|c|c|c|c|c|c|c|}
\hline Molecule & $\boldsymbol{S}_{\text {mol }}$ & $\boldsymbol{\chi}_{\text {mol }}$ & $\boldsymbol{\omega}_{\text {mol }}$ & $\boldsymbol{n H}_{\text {mol }}$ & $\boldsymbol{n C}_{\text {mol }}$ & $\boldsymbol{n N}_{\text {mol }}$ & $\boldsymbol{n N o n H _ { \text { mol } }}$ & $\boldsymbol{n R B _ { \text { mol } }}$ & $\boldsymbol{n H B a _ { \text { mol } }}$ & $\boldsymbol{H y I}_{\text {mol }}$ \\
\hline $\mathbf{2}$ & 0.548 & 0.000 & 0.000 & 0.412 & 0.375 & 0.500 & 0.444 & 0.500 & 0.500 & 0.156 \\
\hline $\mathbf{4}$ & 0.455 & 0.072 & 0.024 & 0.176 & 0.125 & 0.500 & 0.222 & 0.167 & 0.500 & 0.827 \\
\hline $\mathbf{7}$ & 0.103 & 0.916 & 0.554 & 0.353 & 0.375 & 0.000 & 0.333 & 0.333 & 0.000 & 0.118 \\
\hline $\mathbf{9}$ & 0.000 & 0.747 & 0.361 & 0.000 & 0.000 & 0.000 & 0.000 & 0.000 & 0.000 & 1.000 \\
\hline $\mathbf{1 2}$ & 0.151 & 1.000 & 0.654 & 0.706 & 0.750 & 0.000 & 0.667 & 0.500 & 0.000 & 0.010 \\
\hline $\mathbf{1 5}$ & 0.020 & 0.790 & 0.406 & 0.235 & 0.250 & 0.000 & 0.222 & 0.333 & 0.000 & 0.174 \\
\hline $\mathbf{1 6}$ & 0.753 & 0.038 & 0.095 & 1.000 & 1.000 & 0.500 & 1.000 & 1.000 & 0.000 & 0.000 \\
\hline $\mathbf{1 9}$ & 0.938 & 0.476 & 0.552 & 0.706 & 0.750 & 1.000 & 0.778 & 0.667 & 0.500 & 0.047 \\
\hline $\mathbf{2 1}$ & 1.000 & 0.880 & 1.000 & 0.706 & 0.750 & 1.000 & 0.889 & 0.833 & 1.000 & 0.079 \\
\hline $\mathbf{2 3}$ & 0.013 & 0.766 & 0.383 & 0.118 & 0.125 & 0.000 & 0.111 & 0.167 & 0.000 & 0.246 \\
\hline
\end{tabular}

\begin{tabular}{|c|c|c|}
\hline Molecule & $\boldsymbol{M W}_{\text {mol }}$ & $\boldsymbol{A M} \boldsymbol{W}_{\text {mol }}$ \\
\hline $\mathbf{2}$ & 0.398 & 0.247 \\
\hline $\mathbf{4}$ & 0.203 & 0.567 \\
\hline $\mathbf{7}$ & 0.294 & 0.250 \\
\hline $\mathbf{9}$ & 0.000 & 1.000 \\
\hline $\mathbf{1 2}$ & 0.587 & 0.000 \\
\hline $\mathbf{1 5}$ & 0.196 & 0.400 \\
\hline $\mathbf{1 6}$ & 1.000 & 0.044 \\
\hline $\mathbf{1 9}$ & 0.671 & 0.049 \\
\hline $\mathbf{2 1}$ & 0.783 & 0.130 \\
\hline $\mathbf{2 3}$ & 0.098 & 0.625 \\
\hline
\end{tabular}




\section{QSAR Modeling: Node Descriptors}

Table S11. List of node descriptors used in the QSAR models for the prediction of binding free energies.

\begin{tabular}{|c|c|c|c|}
\hline Descriptor & Notation & Unit & Range $^{a}$ \\
\hline distance between 2 binding site metal atoms & $d_{M-M, \text { node }}$ & $\AA$ & $3.584-3.997,3.559-4.017$ \\
\hline NBO partial atomic charge on binding site $\mathrm{M}$ atom $^{b}$ & $q_{M, \text { node }}$ & a.u. & $1.921-2.341$ \\
\hline average NBO partial atomic charge on binding site $\mathrm{M}_{\text {atoms }}{ }^{c}$ & AvqM,node & a.u. & $1.990-2.317$ \\
\hline Wiberg bond index of binding site $\mathrm{M}$ atom ${ }^{b}$ & $B I_{\text {M,node }}$ & & $3.212-4.092$ \\
\hline average Wiberg bond index of binding site $\mathrm{M}$ atoms ${ }^{c}$ & $A v B I_{M, \text { node }}$ & & $3.235-3.903$ \\
\hline NBO population of valence orbitals of binding site $\mathrm{M}$ atom $^{b}$ & ValPop $_{\text {M,node }}$ & & $1.590-2.072$ \\
\hline average NBO population of valence orbitals of binding site $\mathrm{M}$ atoms ${ }^{c}$ & AvValPop ${ }_{M, n o d e}$ & & $1.622-2.000$ \\
\hline experimental ionization potential of $\mathrm{M}^{4+}$ atom & $I P_{M 4+, \text { node }}$ & $\mathrm{eV}$ & $58.000-80.348,58.000-80.348$ \\
\hline experimental electron affinity of $\mathrm{M}^{4+}$ atom & $E A_{M 4+, \text { node }}$ & $\mathrm{eV}$ & $28.648-36.906,28.648-36.906$ \\
\hline experimental hardness of $\mathrm{M}^{4+}$ atom & $\eta_{M 4+, \text { node }}$ & $\mathrm{eV}$ & $14.322-22.965,14.322-22.965$ \\
\hline experimental softness of $\mathrm{M}^{4+}$ atom & $S_{M 4+, \text { node }}$ & $\mathrm{eV}^{-1}$ & $0.044-0.070,0.044-0.070$ \\
\hline experimental electronegativity of $\mathrm{M}^{4+}$ atom & $\chi_{M 4+, \text { node }}$ & $\mathrm{eV}$ & $43.324-57.383,43.324-57.383$ \\
\hline experimental electrophilicity of $\mathrm{M}^{4+}$ atom & $\omega_{M 4+, \text { node }}$ & $\mathrm{eV}$ & $63.947-91.618,63.947-91.618$ \\
\hline atomic number of binding site $\mathrm{M}$ atom & $Z_{M, \text { node }}$ & & $40-90,40-90$ \\
\hline atomic weight of binding site $\mathrm{M}$ atom & AtWt $t_{M, \text { node }}$ & $\mathrm{g} / \mathrm{mol}$ & $91.224-232.040,91.224-232.040$ \\
\hline period in the periodic table of binding site $\mathrm{M}$ atom & $P d_{M, \text { node }}$ & & $5-7,5-7$ \\
\hline covalent radius of binding site $\mathrm{M}$ atom & Cov $R_{M, \text { node }}$ & $\AA$ & $1.75-2.06,1.75-2.06$ \\
\hline total number of linkers per node & $n$ Link $k_{\text {node }}$ & & $6-11,6-11$ \\
\hline linker bite angle & Bite $_{\text {node }}$ & degree & $138.194-157.337,138.127-156.311$ \\
\hline number of electrons in node & nElec $_{\text {node }}$ & & $790-1266,780-1256$ \\
\hline NBO partial atomic charge on binding site $\mu_{3} \mathrm{O}(\mathrm{H})$ atom & $q_{\mu 3 O(H), \text { node }}$ & a.u. & $(-0.974)-(-0.867),(-0.985)-(-0.888)$ \\
\hline NBO partial atomic charge on binding site $\mu_{3}(\mathrm{O}) \mathrm{H}$ atom & $q_{\mu 3(O) H, \text { node }}$ & a.u. & $0.514-0.529,0.509-0.520$ \\
\hline NBO partial atomic charge on binding site $\mu_{3} \mathrm{O}$ atom & $q_{\mu 30, \text { node }}$ & a.u. & $(-1.137)-(-0.916),(-1.163)-(-0.930)$ \\
\hline NBO partial atomic charge on binding site terminal $\mathrm{O}(\mathrm{H})$ atom $^{b}$ & $q_{t o(H), n o d e}$ & a.u. & $(-1.081)-(-0.976)$ \\
\hline NBO partial atomic charge on binding site terminal $(\mathrm{O}) \mathrm{H}$ atom ${ }^{b}$ & $q_{t(O) H, n o d e}$ & a.u. & $0.500-0.508$ \\
\hline bond length of binding site terminal $\mathrm{M}-\mathrm{OH}$ bond ${ }^{b}$ & $B L_{t M-O H, \text { node }}$ & $\AA$ & $2.030-2.210$ \\
\hline Wiberg bond order of binding site terminal $\mathrm{M}-\mathrm{OH}$ bond $^{b}$ & $B O_{t M-O H, \text { node }}$ & & $0.700-0.923$ \\
\hline
\end{tabular}

${ }^{a}$ The values in red correspond to node binding sites with the terminal $-\mathrm{OH}_{2}$ group removed (node-noOH$)_{2}$ ) and values in green correspond to node binding sites with the terminal $-\mathrm{OH}_{2}$ and $-\mathrm{OH}$ groups removed (node-noOH, $\left.\mathrm{OH}\right) .{ }^{b}$ These descriptors were only computed for node-noOH 2 structures. ${ }^{c}$ These descriptors were only computed for nodenoOH $\mathrm{OH}_{2} \mathrm{OH}$ structures. 


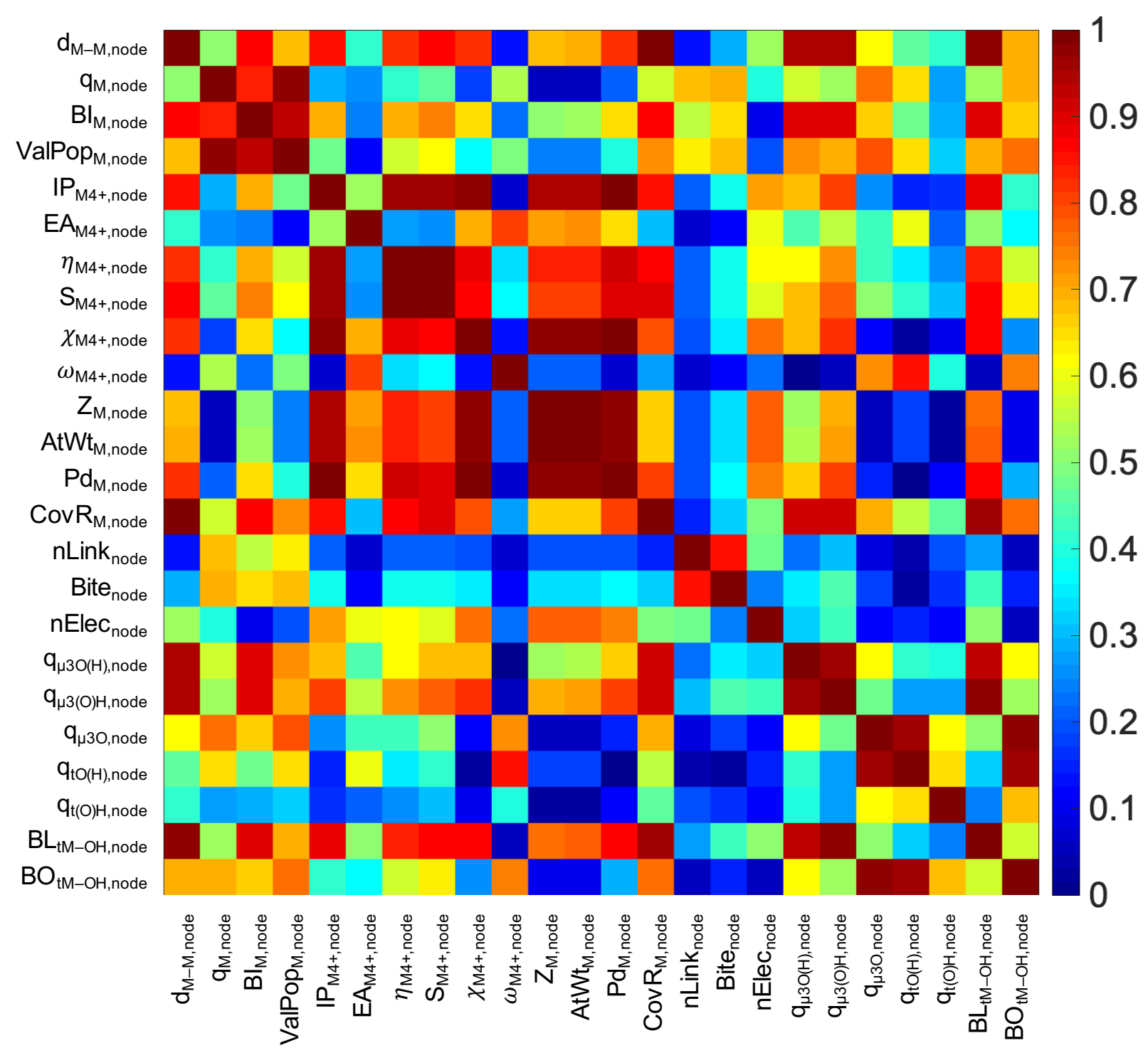

Figure S13. Heatmap of absolute value Pearson's correlation coefficients between node descriptors for the node- $\mathrm{noOH}_{2}$ binding sites. 


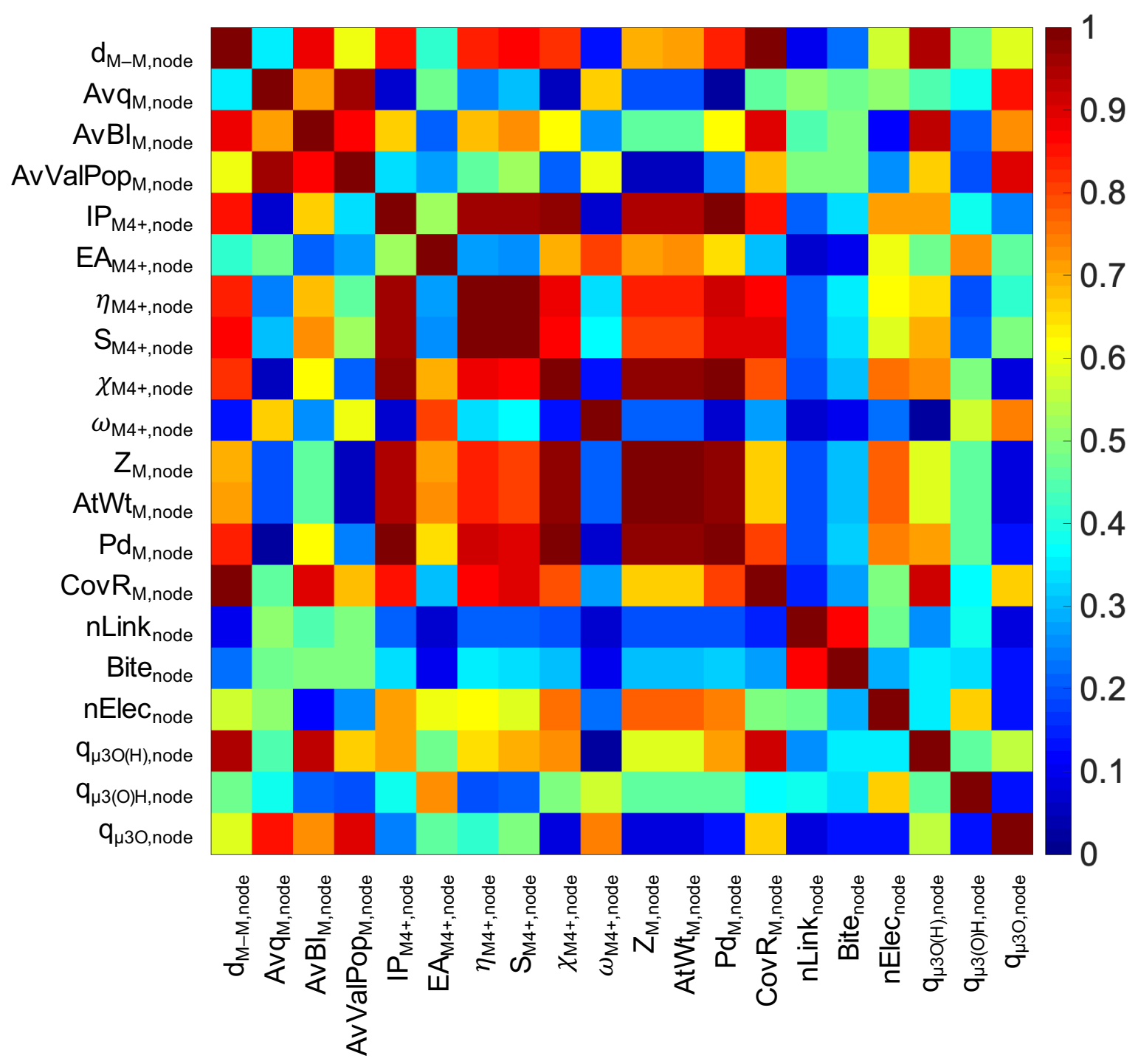

Figure S14. Heatmap of absolute value Pearson's correlation coefficients between node descriptors for the node- $n o \mathrm{OH}_{2}, \mathrm{OH}$ binding sites. 
Table S12. The unscaled values of the node descriptors for the node-noOH $\mathrm{H}_{2}$ binding sites used for QSAR modeling. For descriptor units, refer to Table S11.

\begin{tabular}{|c|c|c|c|c|c|c|c|c|c|}
\hline node-noOH ${ }_{2}$ Site & $\boldsymbol{d}_{M-M, n o d e}$ & $q_{M, n o d e}$ & $B I_{M, \text { node }}$ & ValPop $_{M, \text { node }}$ & IP M4+, node & EAM4+,node & $\eta_{M 4+, \text { node }}$ & $S_{M 4+, \text { node }}$ & $\chi_{M 4+, \text { node }}$ \\
\hline Zr-MOF-808 & 3.600 & 2.056 & 3.667 & 1.876 & 80.348 & 34.418 & 22.965 & 0.044 & 57.383 \\
\hline $\begin{array}{c}\text { Zr-NU-1000 } \\
\text { (large pore) }\end{array}$ & 3.611 & 2.192 & 3.432 & 1.735 & 80.348 & 34.418 & 22.965 & 0.044 & 57.383 \\
\hline $\begin{array}{c}\text { Zr-bi(trans)- } \\
\text { defect UiO-66 }\end{array}$ & 3.617 & 2.233 & 3.378 & 1.700 & 80.348 & 34.418 & 22.965 & 0.044 & 57.383 \\
\hline $\begin{array}{c}\text { Zr-mono- } \\
\text { defect UiO-66 }\end{array}$ & 3.606 & 2.244 & 3.360 & 1.689 & 80.348 & 34.418 & 22.965 & 0.044 & 57.383 \\
\hline $\begin{array}{c}\text { Zr-bi(cis)- } \\
\text { defect UiO-66 }\end{array}$ & 3.623 & 2.203 & 3.414 & 1.725 & 80.348 & 34.418 & 22.965 & 0.044 & 57.383 \\
\hline $\begin{array}{l}\text { Zr-NU-1000 } \\
\text { (c pore) }\end{array}$ & 3.610 & 2.196 & 3.426 & 1.731 & 80.348 & 34.418 & 22.965 & 0.044 & 57.383 \\
\hline $\begin{array}{c}\text { Hf-bi(trans)- } \\
\text { defect UiO-66 }\end{array}$ & 3.595 & 2.341 & 3.212 & 1.590 & 68.370 & 33.370 & 17.500 & 0.057 & 50.870 \\
\hline $\begin{array}{c}\text { Ce-bi(trans)- } \\
\text { defect UiO-66 }\end{array}$ & 3.898 & 2.068 & 3.768 & 1.918 & 65.550 & 36.906 & 14.322 & 0.070 & 51.228 \\
\hline $\begin{array}{c}\text { Th-bi(trans)- } \\
\text { defect UiO-66 }\end{array}$ & 3.990 & 2.224 & 3.781 & 1.781 & 58.000 & 28.648 & 14.676 & 0.068 & 43.324 \\
\hline Hf-MOF-808 & 3.584 & 2.158 & 3.517 & 1.777 & 68.370 & 33.370 & 17.500 & 0.057 & 50.870 \\
\hline Ce-MOF-808 & 3.896 & 1.921 & 4.033 & 2.072 & 65.550 & 36.906 & 14.322 & 0.070 & 51.228 \\
\hline Th-MOF-808 & 3.997 & 2.065 & 4.092 & 1.949 & 58.000 & 28.648 & 14.676 & 0.068 & 43.324 \\
\hline
\end{tabular}


Table S12 (continued).

\begin{tabular}{|c|c|c|c|c|c|c|c|c|c|}
\hline node-noOH ${ }_{2}$ Site & $\omega_{M 4+, \text { node }}$ & $Z_{M, n o d e}$ & $A t W t_{M, n o d e}$ & $P d_{M, \text { node }}$ & Cov $R_{M, n o d e}$ & nLink $_{\text {node }}$ & Bite $_{\text {node }}$ & nElec $_{\text {node }}$ & $q_{\mu 3 O}(H)$, node \\
\hline Zr-MOF-808 & 71.693 & 40 & 91.224 & 5 & 1.75 & 6 & 155.759 & 790 & -0.933 \\
\hline $\begin{array}{c}\text { Zr-NU-1000 } \\
\text { (large pore) }\end{array}$ & 71.693 & 40 & 91.224 & 5 & 1.75 & 8 & 149.637 & 878 & -0.942 \\
\hline $\begin{array}{c}\text { Zr-bi(trans)- } \\
\text { defect UiO-66 }\end{array}$ & 71.693 & 40 & 91.224 & 5 & 1.75 & 10 & 143.714 & 966 & -0.944 \\
\hline $\begin{array}{c}\text { Zr-mono- } \\
\text { defect UiO-66 }\end{array}$ & 71.693 & 40 & 91.224 & 5 & 1.75 & 11 & 142.567 & 1010 & -0.944 \\
\hline $\begin{array}{c}\text { Zr-bi(cis)- } \\
\text { defect UiO-66 }\end{array}$ & 71.693 & 40 & 91.224 & 5 & 1.75 & 10 & 142.463 & 966 & -0.942 \\
\hline $\begin{array}{l}\text { Zr-NU-1000 } \\
\text { (c pore) }\end{array}$ & 71.693 & 40 & 91.224 & 5 & 1.75 & 8 & 138.194 & 878 & -0.943 \\
\hline $\begin{array}{l}\text { Hf-bi(trans)- } \\
\text { defect UiO-66 }\end{array}$ & 73.936 & 72 & 178.490 & 6 & 1.75 & 10 & 143.415 & 1158 & -0.974 \\
\hline $\begin{array}{c}\text { Ce-bi(trans)- } \\
\text { defect UiO-66 }\end{array}$ & 91.618 & 58 & 140.120 & 6 & 2.04 & 10 & 144.622 & 1074 & -0.905 \\
\hline $\begin{array}{c}\text { Th-bi(trans)- } \\
\text { defect UiO-66 }\end{array}$ & 63.947 & 90 & 232.040 & 7 & 2.06 & 10 & 144.456 & 1266 & -0.882 \\
\hline Hf-MOF-808 & 73.936 & 72 & 178.490 & 6 & 1.75 & 6 & 155.763 & 982 & -0.961 \\
\hline Ce-MOF-808 & 91.618 & 58 & 140.120 & 6 & 2.04 & 6 & 156.912 & 898 & -0.902 \\
\hline Th-MOF-808 & 63.947 & 90 & 232.040 & 7 & 2.06 & 6 & 157.337 & 1090 & -0.867 \\
\hline
\end{tabular}


Table S12 (continued).

\begin{tabular}{|c|c|c|c|c|c|c|}
\hline node-noOH ${ }_{2}$ Site & $q_{\mu 3(O) H, n o d e}$ & $q_{\mu 30, \text { node }}$ & $q_{t} O(H)$, node & $q_{t(O) H, \text { node }}$ & $B L_{t M-O H, n o d e}$ & $\mathrm{BO}_{\text {tM-OH,node }}$ \\
\hline Zr-MOF-808 & 0.525 & -1.053 & -1.055 & 0.503 & 2.050 & 0.743 \\
\hline $\begin{array}{c}\text { Zr-NU-1000 } \\
\text { (large pore) }\end{array}$ & 0.526 & -1.064 & -1.049 & 0.504 & 2.030 & 0.754 \\
\hline $\begin{array}{c}\text { Zr-bi(trans)- } \\
\text { defect UiO-66 }\end{array}$ & 0.527 & -1.061 & -1.052 & 0.502 & 2.038 & 0.743 \\
\hline $\begin{array}{c}\text { Zr-mono- } \\
\text { defect UiO-66 }\end{array}$ & 0.528 & -1.062 & -1.052 & 0.501 & 2.043 & 0.737 \\
\hline $\begin{array}{c}\text { Zr-bi(cis)- } \\
\text { defect UiO-66 }\end{array}$ & 0.527 & -1.063 & -1.051 & 0.501 & 2.044 & 0.739 \\
\hline $\begin{array}{l}\text { Zr-NU-1000 } \\
\text { (c pore) }\end{array}$ & 0.527 & -1.062 & -1.049 & 0.503 & 2.035 & 0.751 \\
\hline $\begin{array}{l}\text { Hf-bi(trans)- } \\
\text { defect UiO-66 }\end{array}$ & 0.529 & -1.137 & -1.077 & 0.500 & 2.042 & 0.700 \\
\hline $\begin{array}{l}\text { Ce-bi(trans)- } \\
\text { defect UiO-66 }\end{array}$ & 0.523 & -0.916 & -0.976 & 0.508 & 2.123 & 0.923 \\
\hline $\begin{array}{l}\text { Th-bi(trans)- } \\
\text { defect UiO-66 }\end{array}$ & 0.515 & -1.052 & -1.058 & 0.506 & 2.170 & 0.785 \\
\hline Hf-MOF-808 & 0.527 & -1.129 & -1.081 & 0.501 & 2.053 & 0.701 \\
\hline Ce-MOF-808 & 0.519 & -0.917 & -0.991 & 0.503 & 2.148 & 0.891 \\
\hline Th-MOF-808 & 0.514 & -1.040 & -1.061 & 0.500 & 2.210 & 0.775 \\
\hline
\end{tabular}


Table S13. The unscaled values of the node descriptors for the node-noOH $\mathrm{OH}_{2} \mathrm{Obinding}$ sites used for QSAR modeling. For descriptor units, refer to Table S11.

\begin{tabular}{|c|c|c|c|c|c|c|c|c|c|}
\hline$\underset{\text { Site }}{\text { node-noOH}}$ & $\boldsymbol{d}_{M-M, \text { node }}$ & Avq $q_{M, n o d e}$ & $A v B I_{M, n o d e}$ & AvValPop $_{M, n o d e}$ & IP $_{M 4+, \text { node }}$ & EAM4+, node & $\eta_{M 4+, \text { node }}$ & $S_{M 4+, \text { node }}$ & $\chi_{M 4+, \text { node }}$ \\
\hline Zr-MOF-808 & 3.578 & 2.117 & 3.550 & 1.823 & 80.348 & 34.418 & 22.965 & 0.044 & 57.383 \\
\hline $\begin{array}{c}\mathrm{Zr-NU}-1000 \\
\text { (large pore) }\end{array}$ & 3.567 & 2.193 & 3.418 & 1.744 & 80.348 & 34.418 & 22.965 & 0.044 & 57.383 \\
\hline $\begin{array}{c}\text { Zr-bi(trans)- } \\
\text { defect UiO-66 }\end{array}$ & 3.597 & 2.214 & 3.392 & 1.725 & 80.348 & 34.418 & 22.965 & 0.044 & 57.383 \\
\hline $\begin{array}{c}\text { Zr-mono- } \\
\text { defect UiO-66 }\end{array}$ & 3.588 & 2.211 & 3.396 & 1.729 & 80.348 & 34.418 & 22.965 & 0.044 & 57.383 \\
\hline $\begin{array}{c}\text { Zr-bi(cis)- } \\
\text { defect UiO-66 }\end{array}$ & 3.591 & 2.182 & 3.437 & 1.755 & 80.348 & 34.418 & 22.965 & 0.044 & 57.383 \\
\hline $\begin{array}{l}\text { Zr-NU-1000 } \\
\text { (c pore) }\end{array}$ & 3.563 & 2.181 & 3.437 & 1.756 & 80.348 & 34.418 & 22.965 & 0.044 & 57.383 \\
\hline $\begin{array}{l}\text { Hf-bi(trans)- } \\
\text { defect UiO-66 }\end{array}$ & 3.575 & 2.317 & 3.235 & 1.622 & 68.370 & 33.370 & 17.500 & 0.057 & 50.870 \\
\hline $\begin{array}{l}\text { Ce-bi(trans)- } \\
\text { defect UiO-66 }\end{array}$ & 3.911 & 2.097 & 3.704 & 1.889 & 65.550 & 36.906 & 14.322 & 0.070 & 51.228 \\
\hline $\begin{array}{l}\text { Th-bi(trans)- } \\
\text { defect UiO-66 }\end{array}$ & 4.017 & 2.254 & 3.705 & 1.755 & 58.000 & 28.648 & 14.676 & 0.068 & 43.324 \\
\hline Hf-MOF-808 & 3.559 & 2.218 & 3.400 & 1.723 & 68.370 & 33.370 & 17.500 & 0.057 & 50.870 \\
\hline Ce-MOF-808 & 3.884 & 1.990 & 3.899 & 2.000 & 65.550 & 36.906 & 14.322 & 0.070 & 51.228 \\
\hline Th-MOF-808 & 3.990 & 2.152 & 3.903 & 1.854 & 58.000 & 28.648 & 14.676 & 0.068 & 43.324 \\
\hline
\end{tabular}


Table S13 (continued).

\begin{tabular}{|c|c|c|c|c|c|c|c|c|c|}
\hline $\begin{array}{c}\text { node-noOH } \\
\text { Site }\end{array}$ & $\omega_{M 4+, \text { node }}$ & $Z_{M, n o d e}$ & AtWt $t_{M}$,node & $P d_{M, n o d e}$ & CovR $R_{M, n o d e}$ & nLink $_{\text {node }}$ & Bite $_{\text {node }}$ & nElec $_{\text {node }}$ & $\boldsymbol{q}_{\mu 3} \boldsymbol{O}(\mathrm{H})$, node \\
\hline Zr-MOF-808 & 71.693 & 40 & 91.224 & 5 & 1.75 & 6 & 155.406 & 780 & -0.947 \\
\hline $\begin{array}{c}\text { Zr-NU-1000 } \\
\text { (large pore) }\end{array}$ & 71.693 & 40 & 91.224 & 5 & 1.75 & 8 & 149.531 & 868 & -0.965 \\
\hline $\begin{array}{c}\text { Zr-bi(trans)- } \\
\text { defect UiO-66 }\end{array}$ & 71.693 & 40 & 91.224 & 5 & 1.75 & 10 & 143.341 & 956 & -0.962 \\
\hline $\begin{array}{c}\text { Zr-mono- } \\
\text { defect UiO-66 }\end{array}$ & 71.693 & 40 & 91.224 & 5 & 1.75 & 11 & 142.088 & 1000 & -0.961 \\
\hline $\begin{array}{c}\text { Zr-bi(cis)- } \\
\text { defect UiO-66 }\end{array}$ & 71.693 & 40 & 91.224 & 5 & 1.75 & 10 & 142.144 & 956 & -0.959 \\
\hline $\begin{array}{l}\text { Zr-NU-1000 } \\
\text { (c pore) }\end{array}$ & 71.693 & 40 & 91.224 & 5 & 1.75 & 8 & 138.127 & 868 & -0.964 \\
\hline $\begin{array}{c}\text { Hf-bi(trans)- } \\
\text { defect UiO-66 }\end{array}$ & 73.936 & 72 & 178.490 & 6 & 1.75 & 10 & 143.053 & 1148 & -0.985 \\
\hline $\begin{array}{c}\text { Ce-bi(trans)- } \\
\text { defect UiO-66 }\end{array}$ & 91.618 & 58 & 140.120 & 6 & 2.04 & 10 & 143.581 & 1064 & -0.927 \\
\hline $\begin{array}{l}\text { Th-bi(trans)- } \\
\text { defect UiO-66 }\end{array}$ & 63.947 & 90 & 232.040 & 7 & 2.06 & 10 & 143.308 & 1256 & -0.902 \\
\hline Hf-MOF-808 & 73.936 & 72 & 178.490 & 6 & 1.75 & 6 & 155.416 & 972 & -0.973 \\
\hline Ce-MOF-808 & 91.618 & 58 & 140.120 & 6 & 2.04 & 6 & 155.935 & 888 & -0.922 \\
\hline Th-MOF-808 & 63.947 & 90 & 232.040 & 7 & 2.06 & 6 & 156.311 & 1080 & -0.888 \\
\hline
\end{tabular}


Table S13 (continued).

\begin{tabular}{|c|c|c|}
\hline $\begin{array}{c}\text { node-noOH} \\
\text { Site }\end{array}$ & $q_{\mu 3(0) H, \text { node }}$ & $q_{\mu 30, \text { node }}$ \\
\hline Zr-MOF-808 & 0.520 & -1.076 \\
\hline $\begin{array}{l}\text { Zr-NU-1000 } \\
\text { (large pore) }\end{array}$ & 0.516 & -1.088 \\
\hline $\begin{array}{c}\text { Zr-bi(trans)- } \\
\text { defect UiO-66 }\end{array}$ & 0.514 & -1.084 \\
\hline $\begin{array}{c}\text { Zr-mono- } \\
\text { defect UiO-66 }\end{array}$ & 0.515 & -1.084 \\
\hline $\begin{array}{c}\text { Zr-bi(cis)- } \\
\text { defect UiO-66 }\end{array}$ & 0.515 & -1.085 \\
\hline $\begin{array}{l}\mathrm{Zr}-\mathrm{NU}-1000 \\
\text { (c pore) }\end{array}$ & 0.515 & -1.087 \\
\hline $\begin{array}{c}\text { Hf-bi(trans)- } \\
\text { defect UiO-66 }\end{array}$ & 0.514 & -1.163 \\
\hline $\begin{array}{c}\text { Ce-bi(trans)- } \\
\text { defect UiO-66 }\end{array}$ & 0.518 & -0.935 \\
\hline $\begin{array}{l}\text { Th-bi(trans)- } \\
\text { defect UiO-66 }\end{array}$ & 0.509 & -1.079 \\
\hline Hf-MOF-808 & 0.520 & -1.155 \\
\hline Ce-MOF-808 & 0.517 & -0.930 \\
\hline Th-MOF-808 & 0.511 & -1.070 \\
\hline
\end{tabular}


Table S14. The scaled values of the node descriptors for the node-noOH $\mathrm{H}_{2}$ binding sites used for QSAR modeling.

\begin{tabular}{|c|c|c|c|c|c|c|c|c|c|}
\hline node-noOH ${ }_{2}$ Site & $\boldsymbol{d}_{M-M, n o d e}$ & qM,node & BIM,node & ValPop $_{M, \text { node }}$ & IPM4+, node & EAM4+,node & $\eta_{M 4+, \text { node }}$ & $S_{M 4+, \text { node }}$ & $\chi_{M 4+, \text { node }}$ \\
\hline Zr-MOF-808 & 0.039 & 0.320 & 0.517 & 0.594 & 1.000 & 0.699 & 1.000 & 0.000 & 1.000 \\
\hline $\begin{array}{c}\mathrm{Zr}-\mathrm{NU}-1000 \\
\text { (large pore) }\end{array}$ & 0.064 & 0.646 & 0.250 & 0.301 & 1.000 & 0.699 & 1.000 & 0.000 & 1.000 \\
\hline $\begin{array}{c}\text { Zr-bi(trans)- } \\
\text { defect UiO-66 }\end{array}$ & 0.079 & 0.742 & 0.189 & 0.228 & 1.000 & 0.699 & 1.000 & 0.000 & 1.000 \\
\hline $\begin{array}{c}\text { Zr-mono- } \\
\text { defect UiO-66 }\end{array}$ & 0.052 & 0.768 & 0.168 & 0.206 & 1.000 & 0.699 & 1.000 & 0.000 & 1.000 \\
\hline $\begin{array}{c}\text { Zr-bi(cis)- } \\
\text { defect UiO-66 }\end{array}$ & 0.094 & 0.672 & 0.230 & 0.280 & 1.000 & 0.699 & 1.000 & 0.000 & 1.000 \\
\hline $\begin{array}{l}\text { Zr-NU-1000 } \\
\text { (c pore) }\end{array}$ & 0.062 & 0.655 & 0.243 & 0.293 & 1.000 & 0.699 & 1.000 & 0.000 & 1.000 \\
\hline $\begin{array}{l}\text { Hf-bi(trans)- } \\
\text { defect UiO-66 }\end{array}$ & 0.025 & 1.000 & 0.000 & 0.000 & 0.464 & 0.572 & 0.368 & 0.517 & 0.537 \\
\hline $\begin{array}{l}\text { Ce-bi(trans)- } \\
\text { defect UiO-66 }\end{array}$ & 0.758 & 0.350 & 0.632 & 0.681 & 0.338 & 1.000 & 0.000 & 1.000 & 0.562 \\
\hline $\begin{array}{l}\text { Th-bi(trans)- } \\
\text { defect UiO-66 }\end{array}$ & 0.983 & 0.723 & 0.647 & 0.397 & 0.000 & 0.000 & 0.041 & 0.936 & 0.000 \\
\hline Hf-MOF-808 & 0.000 & 0.564 & 0.346 & 0.389 & 0.464 & 0.572 & 0.368 & 0.517 & 0.537 \\
\hline Ce-MOF-808 & 0.755 & 0.000 & 0.934 & 1.000 & 0.338 & 1.000 & 0.000 & 1.000 & 0.562 \\
\hline Th-MOF-808 & 1.000 & 0.344 & 1.000 & 0.746 & 0.000 & 0.000 & 0.041 & 0.936 & 0.000 \\
\hline
\end{tabular}


Table S14 (continued).

\begin{tabular}{|c|c|c|c|c|c|c|c|c|c|}
\hline node-noOH ${ }_{2}$ Site & $\omega_{M 4+, \text { node }}$ & $Z_{M, n o d e}$ & AtWt & $P d_{M, n o d e}$ & CovR $R_{M, n o d e}$ & nLink $_{\text {node }}$ & Bite $_{\text {node }}$ & nElec $_{\text {node }}$ & $\boldsymbol{q}_{\mu 3} \boldsymbol{O}(\mathrm{H})$, node \\
\hline Zr-MOF-808 & 0.280 & 0.000 & 0.000 & 0.000 & 0.000 & 0.000 & 0.918 & 0.000 & 0.390 \\
\hline $\begin{array}{c}\text { Zr-NU-1000 } \\
\text { (large pore) }\end{array}$ & 0.280 & 0.000 & 0.000 & 0.000 & 0.000 & 0.400 & 0.598 & 0.185 & 0.305 \\
\hline $\begin{array}{c}\text { Zr-bi(trans)- } \\
\text { defect UiO-66 }\end{array}$ & 0.280 & 0.000 & 0.000 & 0.000 & 0.000 & 0.800 & 0.288 & 0.370 & 0.284 \\
\hline $\begin{array}{c}\text { Zr-mono- } \\
\text { defect UiO-66 }\end{array}$ & 0.280 & 0.000 & 0.000 & 0.000 & 0.000 & 1.000 & 0.228 & 0.462 & 0.281 \\
\hline $\begin{array}{c}\text { Zr-bi(cis)- } \\
\text { defect UiO-66 }\end{array}$ & 0.280 & 0.000 & 0.000 & 0.000 & 0.000 & 0.800 & 0.223 & 0.370 & 0.304 \\
\hline $\begin{array}{l}\text { Zr-NU-1000 } \\
\text { (c pore) }\end{array}$ & 0.280 & 0.000 & 0.000 & 0.000 & 0.000 & 0.400 & 0.000 & 0.185 & 0.297 \\
\hline $\begin{array}{l}\text { Hf-bi(trans)- } \\
\text { defect UiO-66 }\end{array}$ & 0.361 & 0.640 & 0.620 & 0.500 & 0.000 & 0.800 & 0.273 & 0.773 & 0.000 \\
\hline $\begin{array}{c}\text { Ce-bi(trans)- } \\
\text { defect UiO-66 }\end{array}$ & 1.000 & 0.360 & 0.347 & 0.500 & 0.935 & 0.800 & 0.336 & 0.597 & 0.644 \\
\hline $\begin{array}{c}\text { Th-bi(trans)- } \\
\text { defect UiO-66 }\end{array}$ & 0.000 & 1.000 & 1.000 & 1.000 & 1.000 & 0.800 & 0.327 & 1.000 & 0.868 \\
\hline Hf-MOF-808 & 0.361 & 0.640 & 0.620 & 0.500 & 0.000 & 0.000 & 0.918 & 0.403 & 0.123 \\
\hline Ce-MOF-808 & 1.000 & 0.360 & 0.347 & 0.500 & 0.935 & 0.000 & 0.978 & 0.227 & 0.678 \\
\hline Th-MOF-808 & 0.000 & 1.000 & 1.000 & 1.000 & 1.000 & 0.000 & 1.000 & 0.630 & 1.000 \\
\hline
\end{tabular}


Table S14 (continued).

\begin{tabular}{|c|c|c|c|c|c|c|}
\hline node-noOH ${ }_{2}$ Site & $q_{\mu 3(O) H, n o d e}$ & $q_{\mu 30, \text { node }}$ & $q_{t} O(H)$, node & $q_{t(O) H, \text { node }}$ & $B L_{t M-O H, n o d e}$ & $\mathrm{BO}_{\text {tM-OH,node }}$ \\
\hline Zr-MOF-808 & 0.766 & 0.381 & 0.245 & 0.429 & 0.115 & 0.195 \\
\hline $\begin{array}{c}\text { Zr-NU-1000 } \\
\text { (large pore) }\end{array}$ & 0.834 & 0.330 & 0.296 & 0.487 & 0.000 & 0.245 \\
\hline $\begin{array}{c}\text { Zr-bi(trans)- } \\
\text { defect UiO-66 }\end{array}$ & 0.886 & 0.343 & 0.275 & 0.342 & 0.046 & 0.194 \\
\hline $\begin{array}{c}\text { Zr-mono- } \\
\text { defect UiO-66 }\end{array}$ & 0.959 & 0.339 & 0.275 & 0.202 & 0.076 & 0.169 \\
\hline $\begin{array}{c}\text { Zr-bi(cis)- } \\
\text { defect UiO-66 }\end{array}$ & 0.868 & 0.333 & 0.285 & 0.169 & 0.078 & 0.175 \\
\hline $\begin{array}{l}\text { Zr-NU-1000 } \\
\text { (c pore) }\end{array}$ & 0.897 & 0.336 & 0.305 & 0.365 & 0.032 & 0.230 \\
\hline $\begin{array}{l}\text { Hf-bi(trans)- } \\
\text { defect UiO-66 }\end{array}$ & 1.000 & 0.000 & 0.033 & 0.000 & 0.070 & 0.000 \\
\hline $\begin{array}{l}\text { Ce-bi(trans)- } \\
\text { defect UiO-66 }\end{array}$ & 0.587 & 1.000 & 1.000 & 1.000 & 0.517 & 1.000 \\
\hline $\begin{array}{l}\text { Th-bi(trans)- } \\
\text { defect UiO-66 }\end{array}$ & 0.065 & 0.384 & 0.213 & 0.766 & 0.777 & 0.380 \\
\hline Hf-MOF-808 & 0.899 & 0.036 & 0.000 & 0.184 & 0.128 & 0.008 \\
\hline Ce-MOF-808 & 0.305 & 0.994 & 0.857 & 0.377 & 0.655 & 0.857 \\
\hline Th-MOF-808 & 0.000 & 0.438 & 0.185 & 0.046 & 1.000 & 0.339 \\
\hline
\end{tabular}


Table S15. The scaled values of the node descriptors for the node-noOH $\mathrm{H}_{2} \mathrm{OH}$ binding sites used for QSAR modeling.

\begin{tabular}{|c|c|c|c|c|c|c|c|c|c|}
\hline $\begin{array}{c}\text { node-noOH} \\
\text { Site }\end{array}$ & $\boldsymbol{d}_{M-M, \text { node }}$ & Avqu,node & AvBI $I_{M, n o d e}$ & $A_{\text {ValPop }}$ M,node & IP $_{M 4+, \text { node }}$ & EAM4+, node & $\eta_{M 4+, \text { node }}$ & $S_{M 4+, \text { node }}$ & $\chi_{M 4+, \text { node }}$ \\
\hline Zr-MOF-808 & 0.041 & 0.389 & 0.472 & 0.531 & 1.000 & 0.699 & 1.000 & 0.000 & 1.000 \\
\hline $\begin{array}{c}\mathrm{Zr-NU}-1000 \\
\text { (large pore) }\end{array}$ & 0.017 & 0.620 & 0.274 & 0.321 & 1.000 & 0.699 & 1.000 & 0.000 & 1.000 \\
\hline $\begin{array}{c}\text { Zr-bi(trans)- } \\
\text { defect UiO-66 }\end{array}$ & 0.082 & 0.686 & 0.235 & 0.272 & 1.000 & 0.699 & 1.000 & 0.000 & 1.000 \\
\hline $\begin{array}{c}\text { Zr-mono- } \\
\text { defect UiO-66 }\end{array}$ & 0.064 & 0.676 & 0.241 & 0.282 & 1.000 & 0.699 & 1.000 & 0.000 & 1.000 \\
\hline $\begin{array}{c}\text { Zr-bi(cis)- } \\
\text { defect UiO-66 }\end{array}$ & 0.069 & 0.588 & 0.302 & 0.351 & 1.000 & 0.699 & 1.000 & 0.000 & 1.000 \\
\hline $\begin{array}{l}\text { Zr-NU-1000 } \\
\text { (c pore) }\end{array}$ & 0.007 & 0.584 & 0.302 & 0.353 & 1.000 & 0.699 & 1.000 & 0.000 & 1.000 \\
\hline $\begin{array}{l}\text { Hf-bi(trans)- } \\
\text { defect UiO-66 }\end{array}$ & 0.034 & 1.000 & 0.000 & 0.000 & 0.464 & 0.572 & 0.368 & 0.517 & 0.537 \\
\hline $\begin{array}{l}\text { Ce-bi(trans)- } \\
\text { defect UiO-66 }\end{array}$ & 0.768 & 0.328 & 0.701 & 0.704 & 0.338 & 1.000 & 0.000 & 1.000 & 0.562 \\
\hline $\begin{array}{l}\text { Th-bi(trans)- } \\
\text { defect UiO-66 }\end{array}$ & 1.000 & 0.808 & 0.703 & 0.350 & 0.000 & 0.000 & 0.041 & 0.936 & 0.000 \\
\hline Hf-MOF-808 & 0.000 & 0.698 & 0.247 & 0.267 & 0.464 & 0.572 & 0.368 & 0.517 & 0.537 \\
\hline Ce-MOF-808 & 0.710 & 0.000 & 0.993 & 1.000 & 0.338 & 1.000 & 0.000 & 1.000 & 0.562 \\
\hline Th-MOF-808 & 0.941 & 0.495 & 1.000 & 0.612 & 0.000 & 0.000 & 0.041 & 0.936 & 0.000 \\
\hline
\end{tabular}


Table S15 (continued).

\begin{tabular}{|c|c|c|c|c|c|c|c|c|c|}
\hline $\begin{array}{c}\text { node-noOH } \\
\text { Site }\end{array}$ & $\omega_{M 4+, \text { node }}$ & $Z_{M, n o d e}$ & AtWt $t_{M}$,node & $P d_{M, n o d e}$ & CovR $R_{M, n o d e}$ & nLink $_{\text {node }}$ & Bite $_{\text {node }}$ & nElec $_{\text {node }}$ & $\boldsymbol{q}_{\mu 3} \boldsymbol{O}(\mathrm{H})$, node \\
\hline Zr-MOF-808 & 0.280 & 0.000 & 0.000 & 0.000 & 0.000 & 0.000 & 0.950 & 0.000 & 0.398 \\
\hline $\begin{array}{c}\text { Zr-NU-1000 } \\
\text { (large pore) }\end{array}$ & 0.280 & 0.000 & 0.000 & 0.000 & 0.000 & 0.400 & 0.627 & 0.185 & 0.212 \\
\hline $\begin{array}{c}\text { Zr-bi(trans)- } \\
\text { defect UiO-66 }\end{array}$ & 0.280 & 0.000 & 0.000 & 0.000 & 0.000 & 0.800 & 0.287 & 0.370 & 0.240 \\
\hline $\begin{array}{c}\text { Zr-mono- } \\
\text { defect UiO-66 }\end{array}$ & 0.280 & 0.000 & 0.000 & 0.000 & 0.000 & 1.000 & 0.218 & 0.462 & 0.247 \\
\hline $\begin{array}{c}\text { Zr-bi(cis)- } \\
\text { defect UiO-66 }\end{array}$ & 0.280 & 0.000 & 0.000 & 0.000 & 0.000 & 0.800 & 0.221 & 0.370 & 0.271 \\
\hline $\begin{array}{c}\text { Zr-NU-1000 } \\
\text { (c pore) }\end{array}$ & 0.280 & 0.000 & 0.000 & 0.000 & 0.000 & 0.400 & 0.000 & 0.185 & 0.220 \\
\hline $\begin{array}{l}\text { Hf-bi(trans)- } \\
\text { defect UiO-66 }\end{array}$ & 0.361 & 0.640 & 0.620 & 0.500 & 0.000 & 0.800 & 0.271 & 0.773 & 0.000 \\
\hline $\begin{array}{c}\text { Ce-bi(trans)- } \\
\text { defect UiO-66 }\end{array}$ & 1.000 & 0.360 & 0.347 & 0.500 & 0.935 & 0.800 & 0.300 & 0.597 & 0.602 \\
\hline $\begin{array}{c}\text { Th-bi(trans)- } \\
\text { defect UiO-66 }\end{array}$ & 0.000 & 1.000 & 1.000 & 1.000 & 1.000 & 0.800 & 0.285 & 1.000 & 0.856 \\
\hline Hf-MOF-808 & 0.361 & 0.640 & 0.620 & 0.500 & 0.000 & 0.000 & 0.951 & 0.403 & 0.128 \\
\hline Ce-MOF-808 & 1.000 & 0.360 & 0.347 & 0.500 & 0.935 & 0.000 & 0.979 & 0.227 & 0.648 \\
\hline Th-MOF-808 & 0.000 & 1.000 & 1.000 & 1.000 & 1.000 & 0.000 & 1.000 & 0.630 & 1.000 \\
\hline
\end{tabular}


Table S15 (continued).

\begin{tabular}{|c|c|c|}
\hline $\begin{array}{c}\text { node-noOH} \\
\text { Site }\end{array}$ & $q_{\mu 3(O) H, n o d e}$ & $q_{\mu 30, \text { node }}$ \\
\hline Zr-MOF-808 & 0.930 & 0.376 \\
\hline $\begin{array}{l}\text { Zr-NU-1000 } \\
\text { (large pore) }\end{array}$ & 0.615 & 0.323 \\
\hline $\begin{array}{c}\text { Zr-bi(trans)- } \\
\text { defect UiO-66 }\end{array}$ & 0.432 & 0.339 \\
\hline $\begin{array}{c}\text { Zr-mono- } \\
\text { defect UiO-66 }\end{array}$ & 0.509 & 0.338 \\
\hline $\begin{array}{c}\text { Zr-bi(cis)- } \\
\text { defect UiO-66 }\end{array}$ & 0.489 & 0.337 \\
\hline $\begin{array}{l}\mathrm{Zr}-\mathrm{NU}-1000 \\
\text { (c pore) }\end{array}$ & 0.557 & 0.328 \\
\hline $\begin{array}{c}\text { Hf-bi(trans)- } \\
\text { defect UiO-66 }\end{array}$ & 0.461 & 0.000 \\
\hline $\begin{array}{c}\text { Ce-bi(trans)- } \\
\text { defect UiO-66 }\end{array}$ & 0.821 & 0.978 \\
\hline $\begin{array}{l}\text { Th-bi(trans)- } \\
\text { defect UiO-66 }\end{array}$ & 0.000 & 0.361 \\
\hline Hf-MOF-808 & 1.000 & 0.036 \\
\hline Ce-MOF-808 & 0.688 & 1.000 \\
\hline Th-MOF-808 & 0.174 & 0.400 \\
\hline
\end{tabular}


QSAR Modeling: Distributions of Binding Free Energies
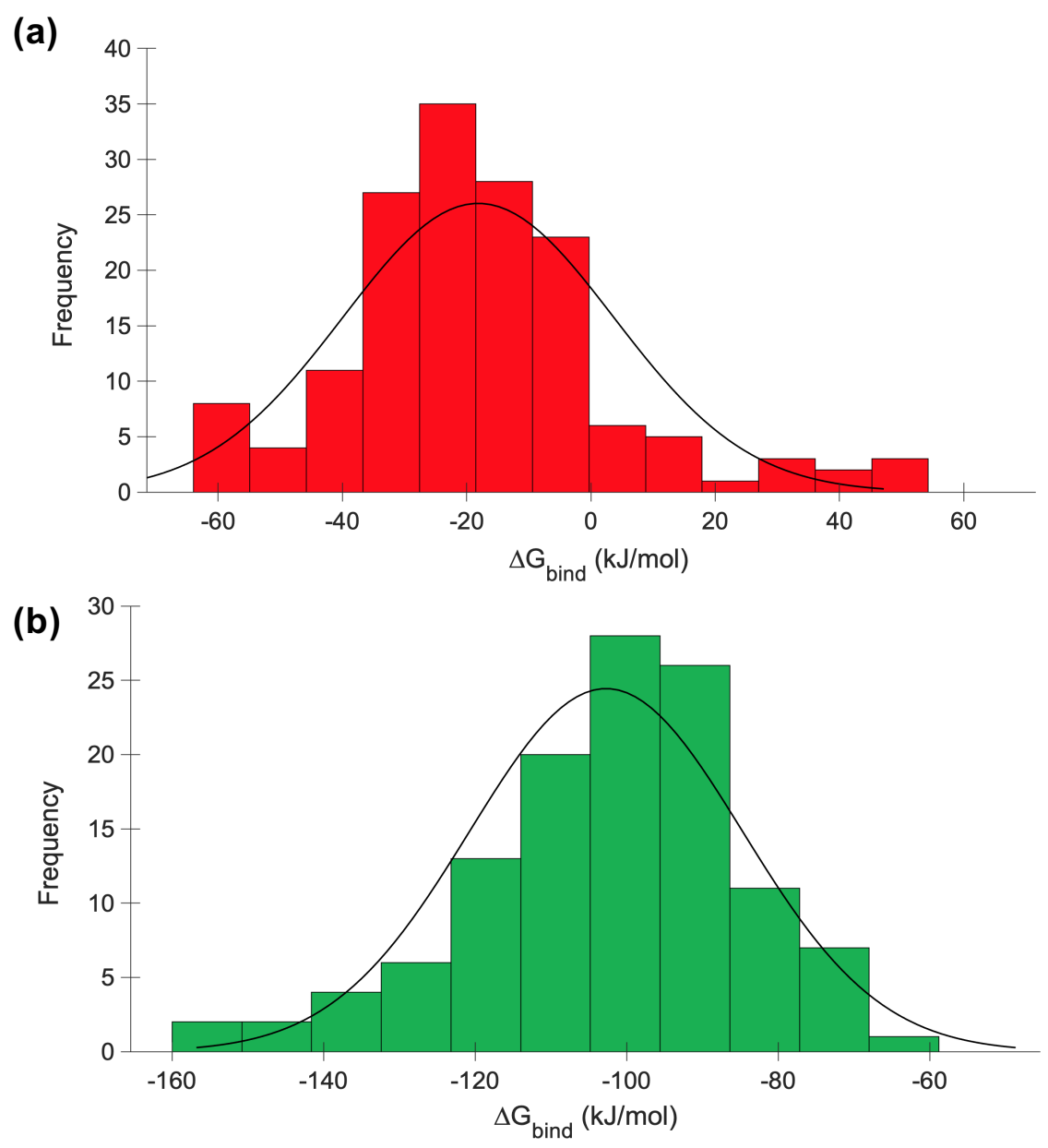

Figure S15. Distribution of binding free energies for (a) the neutral molecules bound to node-noOH $\mathrm{H}_{2}$ sites and (b) the bidentate anions bound to node-noOH${ }_{2}, \mathrm{OH}$ sites. 


\section{QSAR Modeling: Most Important Individual Descriptors}

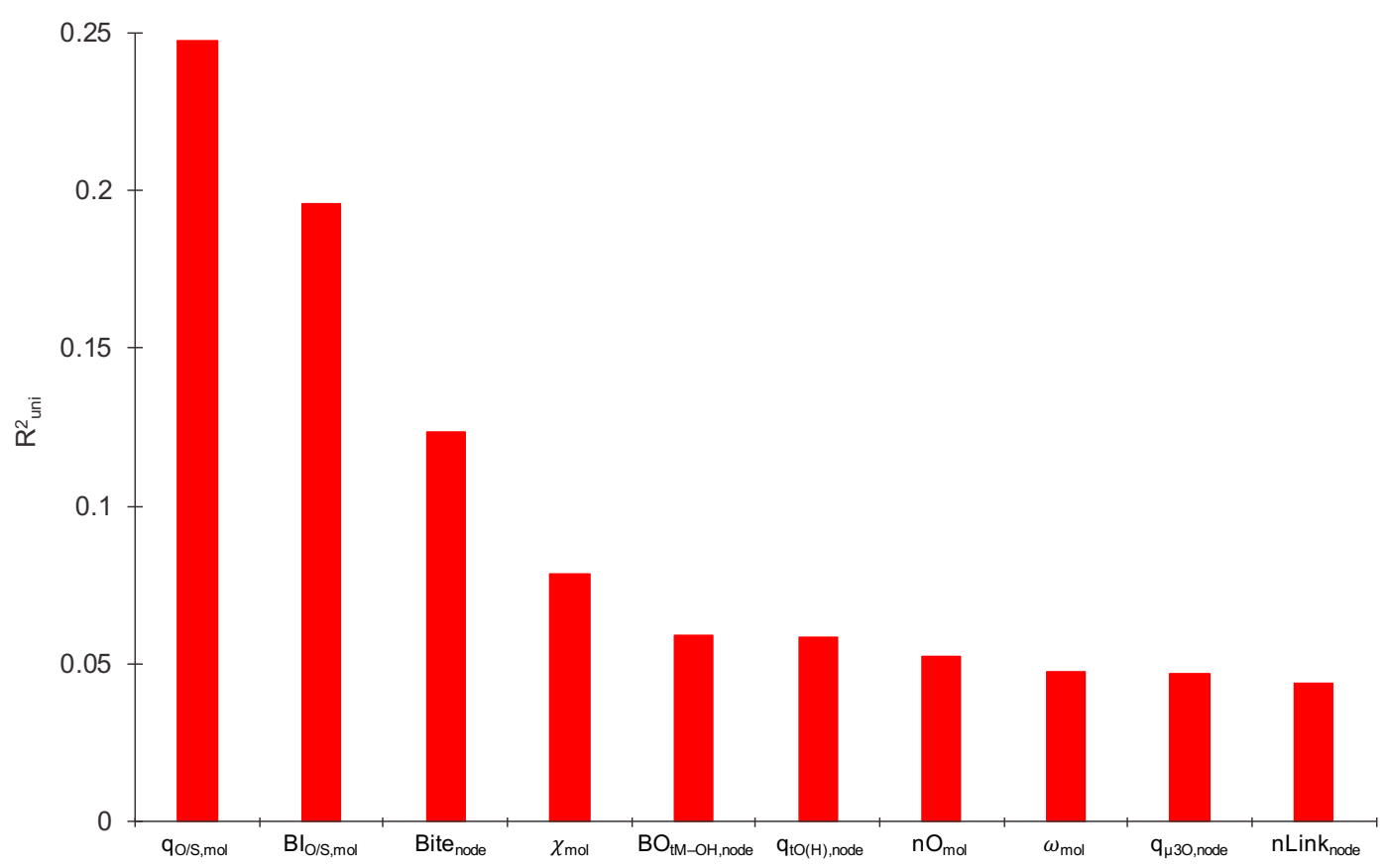

Figure S16. The 10 most important descriptors for describing the binding free energies of neutral molecules to $n o d e-n o \mathrm{OH}_{2}$ sites, as identified by uniparametric coefficients of determination $\left(R_{\text {uni }}^{2}\right)$ for simple linear regression using individual descriptors.

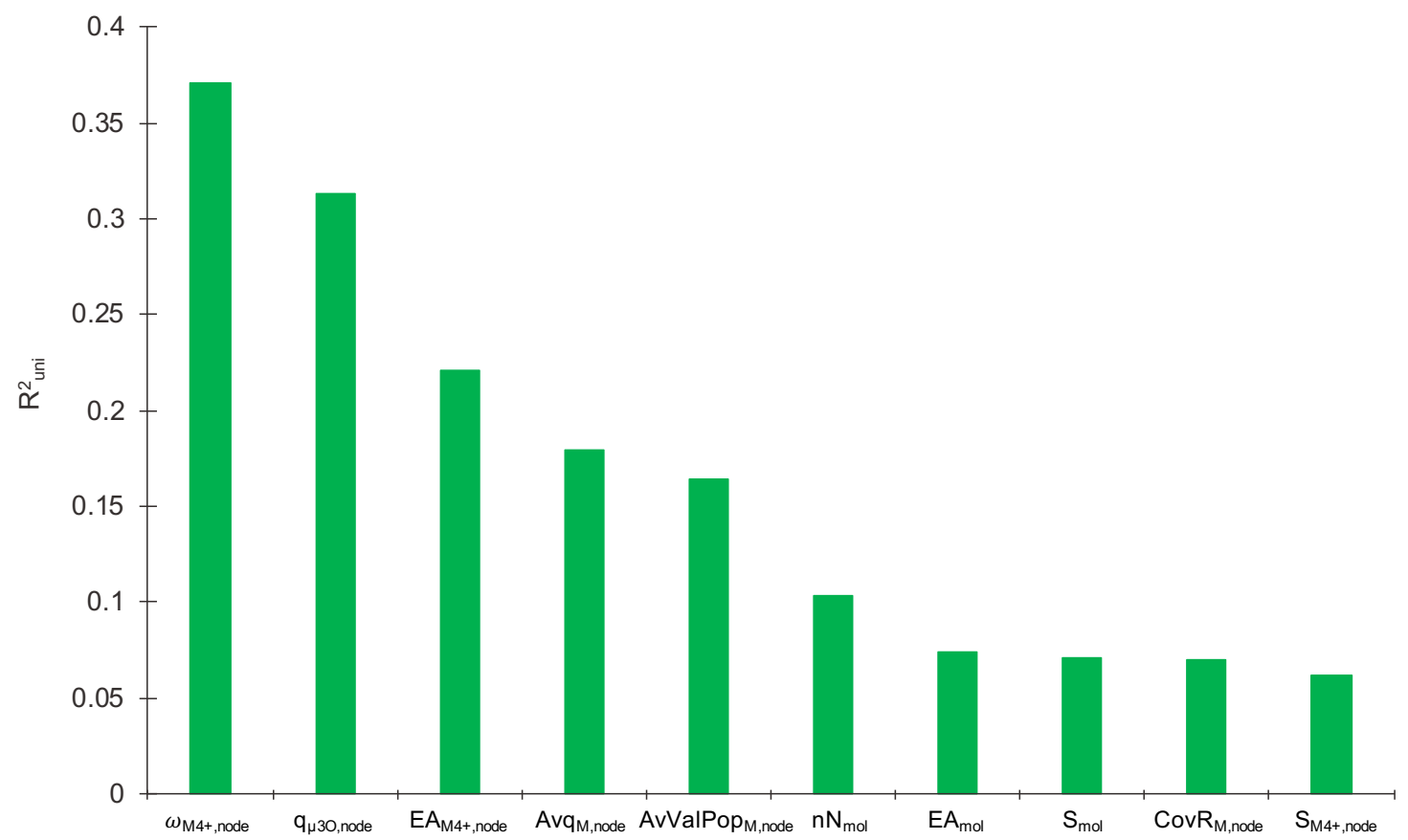

Figure S17. The 10 most important descriptors for describing the binding free energies of bidentate anions to node-noOH, $\mathrm{OH}$ sites, as identified by uniparametric coefficients of determination $\left(R^{2}\right.$ uni $)$ for simple linear regression using individual descriptors. 


\section{QSAR Modeling: Description of Stepwise MLR Algorithm}

As stated in the main text, we used stepwise forward-backward based feature selection combined with MLR using a 95\% confidence interval for development of the multi-parametric QSAR models (for both the neutrals and bidentates datasets). In MATLAB, we utilized the built-in stepwiselm function. This algorithm performs forward selection of descriptors if their corresponding $p$-values are less than 0.05 , then uses backward elimination of the descriptor with the largest $p$-value if any descriptors in the model at the current step have $p$-values higher than 0.10 . Here, the $p$-values are for an F-test of the change in the sum of squared error resulting from the addition or removal of a descriptor. Finally, the algorithm terminates whenever a single step cannot improve the model statistics.

\section{QSAR Modeling: Tests for Statistical Significance}

\section{Training set:}

$$
R_{a d j}^{2}=1-\left(\frac{N_{\text {train }}-1}{N_{\text {train }}-p}\right) \frac{\sum\left(y_{o b s(\text { train })}-y_{\text {pred }(\text { train })}\right)^{2}}{\sum\left(y_{\text {obs }(\text { train })}-\bar{y}_{(\text {train })}\right)^{2}}
$$

In eq $\mathrm{S} 8, y_{\text {obs(train) }}$ are the observed $\Delta G_{\text {bind }}$ responses computed by DFT for the training set molecule-node combinations, $y_{\text {pred(train) }}$ are the responses predicted by the regression equation (developed using the entire training set), $\bar{y}_{(\text {train })}$ is the average response for the training set, and summations are over all training set molecule-node combinations. Further, $N_{\text {train }}$ is the number of training set observations (i.e., the number of molecule-node combinations used to train the QSAR model) and $p$ is the number of regression coefficients (which includes the intercept).

$$
Q_{\text {LOO }}^{2}=1-\frac{\sum\left(y_{\text {obs }(\text { train })}-y_{\text {pred }(\text { train })}\right)^{2}}{\sum\left(y_{\text {obs }(\text { train })}-\bar{y}_{(\text {train })}\right)^{2}}
$$

In eq S9, $y_{\text {obs(train) }}$ are the observed $\Delta G_{\text {bind }}$ responses computed by DFT, $y_{\text {pred(train) }}$ are the responses predicted by the leave-one-out cross-validation (LOO-CV) procedure, $\bar{y}_{(\text {train })}$ is the average response, and summations are over all training set molecule-node combinations. In the LOO-CV procedure, every molecule-node combination is eliminated from the dataset once and then its response variable is predicted using the regression equation derived from the remaining set.

$$
{ }^{C} R_{P}^{2}=R_{n r} \times \sqrt{R_{n r}^{2}-R_{r}^{2}}
$$

The possibility of chance correlation or structural redundancy in the developed QSAR models was measured by the $y$-randomization method. This involved randomizing the training set response values, while leaving the training set descriptor matrix unchanged and repeating the entire process of statistical validation. Using the coefficients of determination for the randomized model $\left(R_{r}^{2}\right)$ and non-random model $\left(R_{n r}^{2}\right)$, the ${ }^{C} R_{P}^{2}$ parameter was calculated using eq S10. If ${ }^{C} R_{P}^{2}>0.5$, then that QSAR model is not generated purely by chance. ${ }^{19}$ 


\section{$\underline{\text { Test set: }}$}

$$
R M S E_{\text {test }}=\sqrt{\frac{\sum\left(y_{\text {obs }(t e s t)}-y_{\text {pred }(\text { test })}\right)^{2}}{N_{\text {test }}}}
$$

In eq S11, $y_{\text {obs (test) }}$ are the observed $\Delta G_{\text {bind }}$ responses computed by DFT for the test set moleculenode combinations, $y_{\text {pred(test) }}$ are the test set responses predicted by the regression equation (developed using the training set), $N_{\text {test }}$ is the number of test set observations, and summations are over all test set molecule-node combinations.

$$
R_{\text {test }}^{2}=1-\frac{\sum\left(y_{o b s(t e s t)}-y_{\text {pred }(t e s t)}\right)^{2}}{\sum\left(y_{\text {obs }(t e s t)}-\bar{y}_{(t e s t)}\right)^{2}}
$$

In eq S12, $\bar{y}_{(t e s t)}$ is the average response for the test set.

$$
\rho_{\text {test }}\left(Y_{\text {obs }(\text { test })}, Y_{\text {pred }(t e s t)}\right)=1-\frac{6 \sum d^{2}}{\mathrm{n}\left(n^{2}-1\right)}
$$

To compute the Spearman's rank correlation coefficient for the test set $\left(\rho_{\text {test }}\right)$, the observed responses and predicted responses are ranked in order of value and then the two columns ( $Y_{o b s(t e s t)}$ and $Y_{\text {pred(test) }}$ ) are compared. In eq S13, $d$ is the difference between the ranks of the two columns and $n$ is the length of each column (i.e., the number of test set observations). 


\section{QSAR Modeling: Variability in Predictive QSAR Model Statistics}
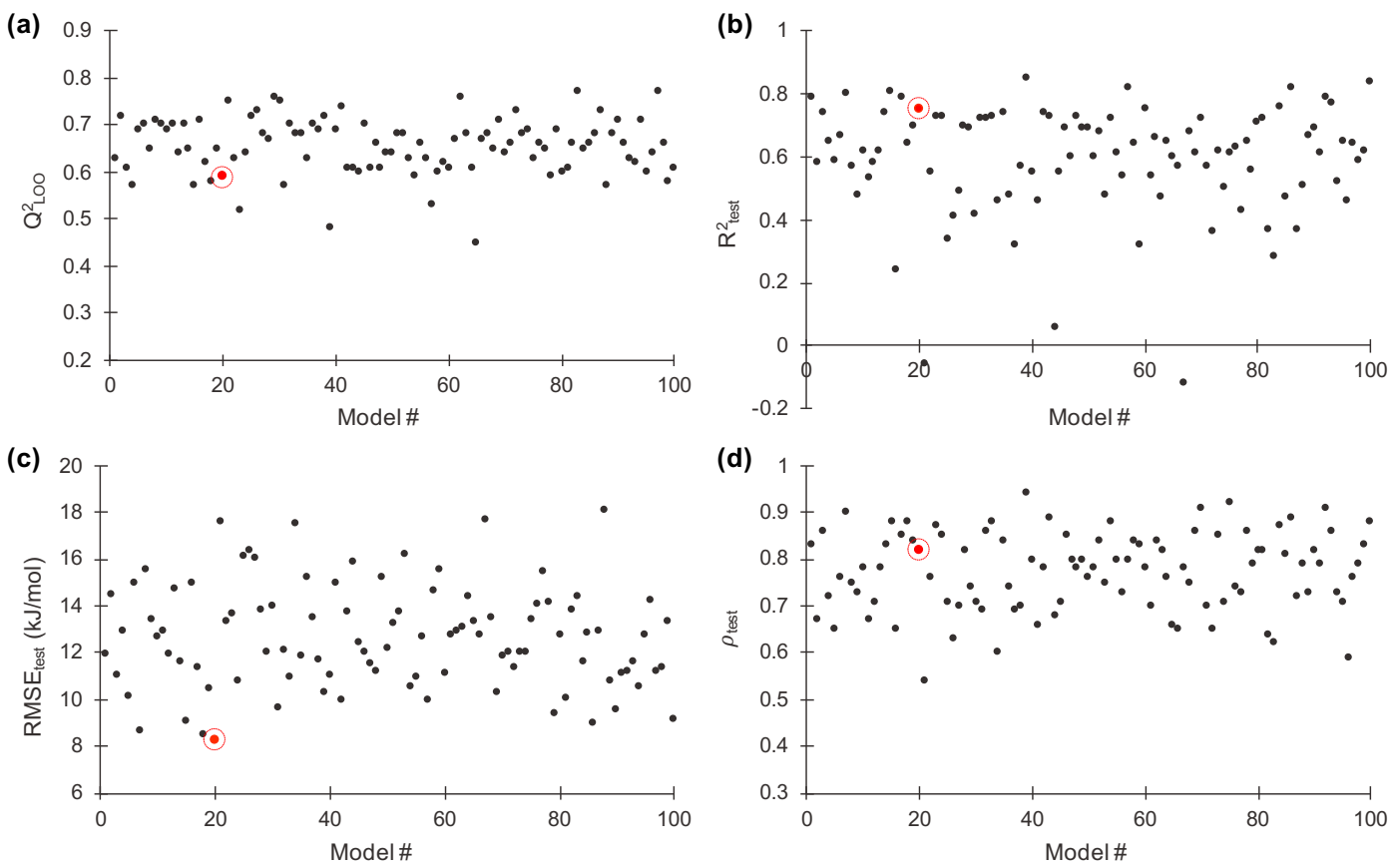

Figure S18. (a) Training set $Q^{2}$ LOO, (b) test set coefficients of determination, (c) test set root mean square errors, and (d) test set Spearman's rank correlation coefficients for the 100 predictive QSAR models generated for the neutrals dataset. Red points indicate the optimal model (i.e., the model with the lowest $\mathrm{RMSE}_{\text {test, }}$ which also satisfies the minimum QSAR criteria of $Q^{2}{ }_{\mathrm{LOO}}>0.5,{ }^{\mathrm{C}} R_{\mathrm{p}}^{2}>0.5$, and $R_{\text {test }}^{2}>0.6$ ).

(a)
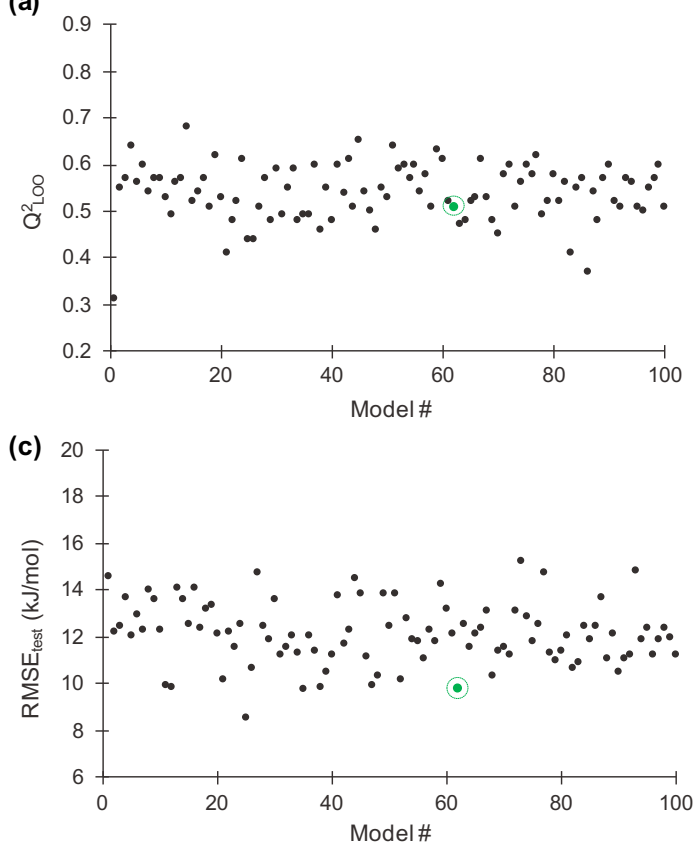

(b)

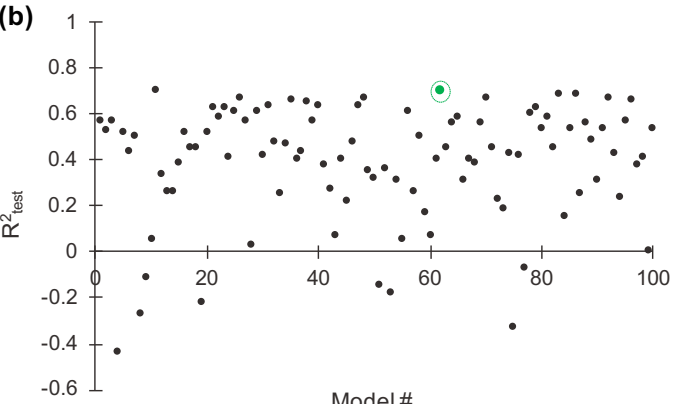

(d)

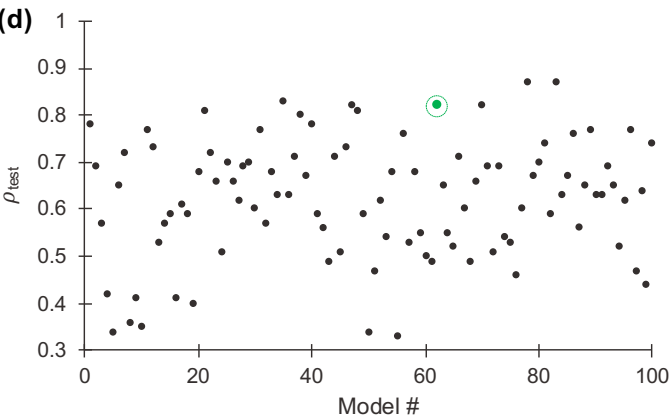

Figure S19. (a) Training set $Q^{2}$ LOO, (b) test set coefficients of determination, (c) test set root mean square errors, and (d) test set Spearman's rank correlation coefficients for the 100 predictive QSAR models generated for the bidentates dataset. Green points indicate the optimal model (i.e., the model with the lowest $\mathrm{RMSE}_{\text {test, }}$ which also satisfies the minimum QSAR criteria of $Q^{2}{ }_{\mathrm{LOO}}>0.5,{ }^{\mathrm{C}} R_{\mathrm{p}}^{2}>0.5$, and $R_{\text {test }}^{2}>0.6$ ). 


\section{QSAR Modeling: Leverages and Standardized Residuals}

As stated in the main text, leverage $(h)$ is a measure of the influence of a molecule-node combination's properties on a regression model. Leverages for individual molecule-node combinations within the training and test sets are calculated as the diagonals of the hat matrices $(H)$, defined as:

$$
\begin{aligned}
& H_{\text {train }}=X_{\text {train }}\left(X_{\text {train }}^{T} X_{\text {train }}\right)^{-1} X_{\text {train }}^{T} \\
& H_{\text {test }}=X_{\text {test }}\left(X_{\text {train }}^{T} X_{\text {train }}\right)^{-1} X_{\text {test }}^{T}
\end{aligned}
$$

where $X_{\text {train }}$ and $X_{\text {test }}$ are the design matrices containing the molecular/node descriptors for the training and test sets, respectively. More specifically, each row in the design matrices contains the 49 (42) scaled descriptor values for an individual molecule-node combination in the neutrals (bidentates) dataset.

The standardized residuals for response $i\left(s t_{i}\right)$ are computed as raw residuals $\left(r_{i}\right)$ divided by their estimated standard deviation:

$$
s t_{i}=\frac{r_{i}}{\sqrt{M S E\left(1-h_{i i}\right)}}=\frac{y_{o b s, i}-y_{\text {pred }, i}}{\sqrt{M S E\left(1-h_{i i}\right)}}
$$

where $M S E$ is the mean squared error and $h_{i i}$ is the leverage value for $\Delta G_{b i n d}$ response $i$. 


\section{QSAR Modeling: Optimal Predictive QSAR Model for Neutrals Dataset}

Table S16. Predictions, residuals, and leverages for training and test set molecule-node combinations using the optimal predictive QSAR model for the neutrals dataset (eq 9 in the main text).

\begin{tabular}{|c|c|c|c|c|c|c|}
\hline Node/Molecule & Set & $\begin{array}{c}\text { Observation } \\
(\mathbf{k J} / \mathbf{m o l})\end{array}$ & $\begin{array}{c}\text { Prediction } \\
(\mathrm{kJ} / \mathrm{mol})\end{array}$ & $\begin{array}{c}\text { Raw Residual } \\
(\mathbf{k J} / \mathbf{m o l})\end{array}$ & $\begin{array}{c}\text { Standardized } \\
\text { Residual }\end{array}$ & Leverage \\
\hline Zr-NU-1000 (c pore)/18 & Training & -62.23 & -57.57 & -4.66 & -0.52 & 0.104 \\
\hline Zr-bi(trans)-defect UiO-66/17 & Training & 53.55 & 47.49 & 6.06 & 0.70 & 0.157 \\
\hline Zr-mono-defect UiO-66/24 & Training & -22.86 & -21.82 & -1.04 & -0.11 & 0.055 \\
\hline Zr-MOF-808/3 & Training & 10.75 & 1.24 & 9.51 & 1.04 & 0.060 \\
\hline Ce-bi(trans)-defect UiO-66/3 & Training & -30.60 & -32.02 & 1.42 & 0.16 & 0.084 \\
\hline Zr-mono-defect UiO-66/17 & Training & 31.15 & 47.45 & -16.30 & -2.08 & 0.305 \\
\hline Hf-bi(trans)-defect UiO-66/22 & Training & -10.52 & -10.34 & -0.18 & -0.02 & 0.144 \\
\hline Zr-NU-1000 (c pore)/20 & Training & -61.17 & -61.84 & 0.67 & 0.08 & 0.147 \\
\hline Th-bi(trans)-defect UiO-66/14 & Training & -45.92 & -42.56 & -3.36 & -0.40 & 0.193 \\
\hline Hf-MOF-808/14 & Training & 14.21 & 3.47 & 10.74 & 1.27 & 0.191 \\
\hline Zr-mono-defect UiO-66/13 & Training & -24.21 & -13.70 & -10.51 & -1.18 & 0.100 \\
\hline Hf-bi(trans)-defect UiO-66/ $\mathrm{H}_{2} \mathrm{O}$ & Training & -35.37 & -44.01 & 8.64 & 1.08 & 0.271 \\
\hline Ce-bi(trans)-defect UiO-66/20 & Training & -48.02 & -49.29 & 1.27 & 0.15 & 0.170 \\
\hline Zr-NU-1000 (c pore)/13 & Training & -15.07 & -38.95 & 23.88 & 2.69 & 0.105 \\
\hline Zr-MOF-808/11 & Training & -2.17 & -5.79 & 3.62 & 0.40 & 0.058 \\
\hline Zr-NU-1000 (large pore)/18 & Training & -18.65 & -20.35 & 1.70 & 0.18 & 0.042 \\
\hline Hf-MOF-808/17 & Training & 37.89 & 37.30 & 0.59 & 0.08 & 0.298 \\
\hline Ce-bi(trans)-defect UiO-66/24 & Training & -28.88 & -30.25 & 1.37 & 0.15 & 0.092 \\
\hline Zr-NU-1000 (large pore)/8 & Training & -2.77 & -4.76 & 1.99 & 0.22 & 0.034 \\
\hline Zr-bi(cis)-defect UiO-66/14 & Training & -19.44 & -36.46 & 17.02 & 1.95 & 0.138 \\
\hline Th-MOF-808/18 & Training & -22.03 & -13.76 & -8.28 & -0.94 & 0.129 \\
\hline Zr-bi(trans)-defect UiO-66/24 & Training & -22.74 & -19.85 & -2.89 & -0.31 & 0.036 \\
\hline Zr-MOF-808/13 & Training & 15.91 & 8.56 & 7.35 & 0.81 & 0.074 \\
\hline Hf-MOF- $808 / 20$ & Training & -20.04 & -19.72 & -0.32 & -0.04 & 0.136 \\
\hline Hf-bi(trans)-defect UiO-66/20 & Training & -41.13 & -31.53 & -9.60 & -1.12 & 0.172 \\
\hline Ce-bi(trans)-defect UiO-66/13 & Training & -30.32 & -31.57 & 1.25 & 0.14 & 0.125 \\
\hline Ce-MOF-808/11 & Training & -20.18 & -23.18 & 3.00 & 0.33 & 0.073 \\
\hline Zr-NU-1000 (c pore) $/ 8$ & Training & -27.65 & -43.81 & 16.16 & 1.82 & 0.105 \\
\hline Hf-MOF-808/3 & Training & 17.40 & -5.31 & 22.71 & 2.50 & 0.068 \\
\hline Zr-NU-1000 (large pore)/17 & Training & 52.11 & 51.55 & 0.56 & 0.07 & 0.213 \\
\hline Hf-bi(trans)-defect UiO-66/24 & Training & -29.28 & -28.60 & -0.68 & -0.07 & 0.075 \\
\hline Th-bi(trans)-defect UiO-66/18 & Training & -28.85 & -33.37 & 4.52 & 0.50 & 0.082 \\
\hline Ce-MOF-808/22 & Training & -18.32 & -23.96 & 5.64 & 0.62 & 0.064 \\
\hline
\end{tabular}




\begin{tabular}{|c|c|c|c|c|c|c|}
\hline Zr-bi(trans)-defect UiO-66/20 & Training & -34.95 & -30.29 & -4.66 & -0.52 & 0.093 \\
\hline Hf-bi(trans)-defect UiO-66/13 & Training & -33.49 & -19.33 & -14.16 & -1.58 & 0.089 \\
\hline Ce-MOF-808/17 & Training & -10.26 & -22.97 & 12.71 & 1.58 & 0.265 \\
\hline Zr-NU-1000 (large pore)/24 & Training & 10.52 & -6.76 & 17.28 & 1.88 & 0.041 \\
\hline Zr-NU-1000 (large pore)/13 & Training & -17.51 & 0.01 & -17.52 & -1.91 & 0.046 \\
\hline Zr-NU-1000 (large pore)/20 & Training & -26.13 & -24.56 & -1.57 & -0.18 & 0.095 \\
\hline Ce-MOF-808/14 & Training & -26.85 & -21.86 & -4.99 & -0.60 & 0.226 \\
\hline Ce-MOF-808/13 & Training & -23.03 & -16.49 & -6.54 & -0.73 & 0.092 \\
\hline Zr-bi(cis)-defect UiO-66/13 & Training & -31.11 & -19.47 & -11.64 & -1.29 & 0.073 \\
\hline Hf-bi(trans)-defect UiO-66/17 & Training & 42.31 & 42.88 & -0.57 & -0.07 & 0.321 \\
\hline Th-bi(trans)-defect UiO-66/ $\mathrm{H}_{2} \mathrm{O}$ & Training & -38.22 & -46.35 & 8.13 & 0.94 & 0.157 \\
\hline Th-bi(trans)-defect UiO-66/6 & Training & -12.66 & -15.31 & 2.65 & 0.30 & 0.112 \\
\hline Zr-NU-1000 (c pore)/6 & Training & -53.29 & -41.87 & -11.42 & -1.30 & 0.128 \\
\hline Ce-bi(trans)-defect UiO-66/14 & Training & -59.86 & -61.84 & 1.98 & 0.27 & 0.407 \\
\hline Zr-bi(cis)-defect UiO-66/17 & Training & 46.79 & 32.95 & 13.84 & 1.63 & 0.188 \\
\hline Hf-bi(trans)-defect UiO-66/18 & Training & -33.49 & -28.99 & -4.50 & -0.50 & 0.095 \\
\hline Hf-bi(trans)-defect UiO-66/11 & Training & -13.15 & -12.71 & -0.44 & -0.05 & 0.082 \\
\hline Th-MOF-808/3 & Training & -20.22 & -21.20 & 0.98 & 0.11 & 0.091 \\
\hline Ce-bi(trans)-defect UiO-66/ $\mathrm{H}_{2} \mathrm{O}$ & Training & -31.97 & -38.33 & 6.36 & 0.72 & 0.124 \\
\hline Zr-bi(cis)-defect UiO-66/20 & Training & -22.08 & -35.62 & 13.54 & 1.52 & 0.099 \\
\hline $\mathrm{Zr}$-bi(trans)-defect UiO-66/ $\mathrm{H}_{2} \mathrm{O}$ & Training & -29.93 & -34.33 & 4.40 & 0.51 & 0.142 \\
\hline Zr-bi(trans)-defect UiO-66/22 & Training & -4.41 & -11.46 & 7.05 & 0.78 & 0.066 \\
\hline Ce-MOF-808/18 & Training & -32.30 & -28.09 & -4.21 & -0.47 & 0.105 \\
\hline Hf-bi(trans)-defect UiO-66/1 & Training & -4.56 & -9.36 & 4.80 & 0.54 & 0.114 \\
\hline Th-bi(trans)-defect UiO-66/24 & Training & -60.15 & -34.47 & -25.68 & -2.87 & 0.095 \\
\hline Hf-MOF-808/8 & Training & -5.76 & -3.48 & -2.28 & -0.25 & 0.062 \\
\hline Zr-bi(cis)-defect UiO-66/24 & Training & -36.40 & -27.68 & -8.72 & -0.95 & 0.044 \\
\hline Ce-MOF-808/3 & Training & -21.76 & -17.54 & -4.22 & -0.46 & 0.067 \\
\hline Zr-NU-1000 (c pore) $/ \mathrm{H}_{2} \mathrm{O}$ & Training & -61.08 & -58.70 & -2.38 & -0.29 & 0.213 \\
\hline Zr-mono-defect UiO-66/18 & Training & -22.41 & -26.05 & 3.64 & 0.40 & 0.069 \\
\hline Zr-bi(trans)-defect UiO-66/14 & Training & -39.10 & -31.65 & -7.45 & -0.85 & 0.123 \\
\hline Zr-MOF-808/22 & Training & -5.32 & -8.56 & 3.24 & 0.36 & 0.064 \\
\hline Ce-bi(trans)-defect UiO-66/11 & Training & -30.19 & -33.80 & 3.61 & 0.42 & 0.148 \\
\hline Zr-mono-defect UiO-66/20 & Training & -42.32 & -27.76 & -14.56 & -1.65 & 0.120 \\
\hline Zr-bi(cis)-defect UiO-66/8 & Training & -16.97 & -24.31 & 7.34 & 0.80 & 0.050 \\
\hline Zr-NU-1000 (c pore)/22 & Training & -45.37 & -44.48 & -0.89 & -0.10 & 0.134 \\
\hline Hf-MOF-808/22 & Training & -5.23 & -6.82 & 1.59 & 0.18 & 0.118 \\
\hline Hf-MOF-808/18 & Training & -11.96 & -13.86 & 1.90 & 0.21 & 0.084 \\
\hline Zr-bi(cis)-defect UiO-66/3 & Training & -13.59 & -27.92 & 14.33 & 1.56 & 0.046 \\
\hline
\end{tabular}




\begin{tabular}{|c|c|c|c|c|c|c|}
\hline Zr-NU-1000 (c pore) $/ 3$ & Training & -57.65 & -46.09 & -11.56 & -1.31 & 0.116 \\
\hline $\mathrm{Zr}-\mathrm{MOF}-808 / \mathrm{H}_{2} \mathrm{O}$ & Training & -7.91 & -8.47 & 0.56 & 0.06 & 0.123 \\
\hline Zr-NU-1000 (large pore)/6 & Training & -2.67 & -4.58 & 1.91 & 0.21 & 0.077 \\
\hline Zr-mono-defect UiO-66/11 & Training & -10.84 & -8.52 & -2.32 & -0.27 & 0.146 \\
\hline Zr-bi(trans)-defect UiO-66/1 & Training & 4.59 & -9.67 & 14.26 & 1.58 & 0.075 \\
\hline Ce-bi(trans)-defect UiO-66/6 & Training & -31.15 & -29.18 & -1.97 & -0.22 & 0.083 \\
\hline Th-bi(trans)-defect UiO-66/20 & Training & -32.48 & -33.30 & 0.82 & 0.09 & 0.109 \\
\hline Th-bi(trans)-defect UiO-66/1 & Training & -18.98 & -14.85 & -4.13 & -0.47 & 0.113 \\
\hline Zr-NU-1000 (large pore)/22 & Training & -5.00 & -7.21 & 2.21 & 0.24 & 0.076 \\
\hline Ce-bi(trans)-defect UiO-66/17 & Training & 2.90 & 7.58 & -4.68 & -0.68 & 0.466 \\
\hline Zr-MOF-808/17 & Training & 32.39 & 37.92 & -5.53 & -0.68 & 0.251 \\
\hline Ce-MOF-808/8 & Training & -24.64 & -17.22 & -7.42 & -0.82 & 0.063 \\
\hline Zr-bi(cis)-defect UiO-66/22 & Training & -24.18 & -17.96 & -6.22 & -0.68 & 0.056 \\
\hline Zr-NU-1000 (large pore) $/ \mathrm{H}_{2} \mathrm{O}$ & Training & -28.06 & -20.24 & -7.82 & -0.89 & 0.132 \\
\hline Hf-bi(trans)-defect UiO-66/8 & Training & -17.57 & -25.26 & 7.69 & 0.85 & 0.067 \\
\hline Th-MOF-808/20 & Training & -24.76 & -17.13 & -7.63 & -0.93 & 0.238 \\
\hline Zr-bi(trans)-defect UiO-66/6 & Training & -17.01 & -7.88 & -9.13 & -1.01 & 0.072 \\
\hline $\mathrm{Zr}$-mono-defect $\mathrm{UiO}-66 / \mathrm{H}_{2} \mathrm{O}$ & Training & -40.93 & -36.06 & -4.87 & -0.58 & 0.198 \\
\hline Zr-bi(cis)-defect UiO-66/6 & Training & -20.29 & -14.49 & -5.80 & -0.64 & 0.061 \\
\hline Zr-mono-defect UiO-66/8 & Training & -4.21 & -18.34 & 14.13 & 1.56 & 0.071 \\
\hline Zr-MOF-808/18 & Training & -17.53 & -13.45 & -4.08 & -0.45 & 0.076 \\
\hline Th-MOF- $808 / \mathrm{H}_{2} \mathrm{O}$ & Training & -21.56 & -23.25 & 1.69 & 0.20 & 0.167 \\
\hline Th-MOF-808/24 & Training & -17.00 & -22.41 & 5.41 & 0.61 & 0.101 \\
\hline Th-bi(trans)-defect UiO-66/8 & Training & -34.53 & -32.26 & -2.27 & -0.25 & 0.091 \\
\hline Th-MOF-808/22 & Training & -14.84 & -10.80 & -4.04 & -0.45 & 0.099 \\
\hline Zr-NU-1000 (large pore) $/ 3$ & Training & -10.80 & -6.98 & -3.82 & -0.41 & 0.035 \\
\hline Zr-bi(trans)-defect UiO-66/13 & Training & -12.81 & -12.27 & -0.54 & -0.06 & 0.063 \\
\hline Th-MOF-808/8 & Training & -24.14 & -19.80 & -4.34 & -0.48 & 0.086 \\
\hline Hf-MOF-808/13 & Training & 18.72 & 3.52 & 15.20 & 1.68 & 0.076 \\
\hline Ce-MOF- $808 / \mathrm{H}_{2} \mathrm{O}$ & Training & -28.77 & -16.30 & -12.47 & -1.39 & 0.084 \\
\hline Zr-bi(trans)-defect UiO-66/3 & Training & -21.40 & -20.40 & -1.00 & -0.11 & 0.037 \\
\hline Zr-bi(cis)-defect UiO-66/18 & Training & -45.77 & -32.93 & -12.84 & -1.41 & 0.056 \\
\hline Zr-mono-defect UiO-66/3 & Training & -13.37 & -22.43 & 9.06 & 0.99 & 0.061 \\
\hline Zr-NU-1000 (large pore)/14 & Training & -30.54 & -15.14 & -15.40 & -1.73 & 0.104 \\
\hline Hf-MOF-808/1 & Training & -0.40 & -12.03 & 11.63 & 1.32 & 0.117 \\
\hline Zr-NU-1000 (c pore)/11 & Training & -40.63 & -45.35 & 4.72 & 0.53 & 0.085 \\
\hline Zr-NU-1000 (c pore)/17 & Training & -1.96 & 9.83 & -11.79 & -1.40 & 0.196 \\
\hline Zr-MOF-808/8 & Training & -6.35 & 2.78 & -9.13 & -1.00 & 0.058 \\
\hline Th-bi(trans)-defect UiO-66/17 & Training & 33.93 & 21.64 & 12.29 & 1.60 & 0.335 \\
\hline
\end{tabular}




\begin{tabular}{|c|c|c|c|c|c|c|}
\hline Th-MOF-808/11 & Training & -5.02 & -10.14 & 5.12 & 0.57 & 0.078 \\
\hline Zr-NU-1000 (large pore)/1 & Training & 1.87 & -9.06 & 10.93 & 1.20 & 0.066 \\
\hline Ce-MOF-808/6 & Training & -18.51 & -23.94 & 5.43 & 0.60 & 0.061 \\
\hline Zr-mono-defect UiO-66/22 & Training & -1.06 & -8.47 & 7.41 & 0.82 & 0.083 \\
\hline Th-bi(trans)-defect UiO-66/11 & Training & -19.34 & -20.56 & 1.22 & 0.14 & 0.107 \\
\hline Ce-bi(trans)-defect UiO-66/8 & Training & -27.51 & -30.26 & 2.75 & 0.31 & 0.081 \\
\hline Hf-MOF-808/24 & Training & -16.35 & -6.41 & -9.94 & -1.10 & 0.081 \\
\hline Zr-MOF-808/24 & Training & -23.57 & 0.22 & -23.79 & -2.62 & 0.067 \\
\hline Ce-MOF-808/20 & Training & -29.83 & -33.40 & 3.57 & 0.42 & 0.190 \\
\hline Hf-bi(trans)-defect UiO-66/3 & Training & -29.49 & -29.02 & -0.47 & -0.05 & 0.071 \\
\hline Zr-NU-1000 (large pore)/11 & Training & -2.78 & -8.05 & 5.27 & 0.57 & 0.036 \\
\hline Th-MOF-808/17 & Training & -22.68 & -14.72 & -7.96 & -1.26 & 0.548 \\
\hline Th-bi(trans)-defect UiO-66/22 & Training & -17.42 & -18.53 & 1.11 & 0.12 & 0.110 \\
\hline 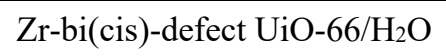 & Training & -54.46 & -40.17 & -14.29 & -1.62 & 0.120 \\
\hline Th-MOF-808/14 & Test & -12.52 & -3.94 & -8.58 & -1.13 & 0.152 \\
\hline Zr-mono-defect UiO-66/6 & Test & -12.54 & -4.46 & -8.08 & -1.03 & 0.095 \\
\hline Zr-NU-1000 (c pore)/1 & Test & -30.26 & -46.51 & 16.25 & 2.08 & 0.102 \\
\hline Ce-MOF-808/1 & Test & -17.88 & -29.29 & 11.41 & 1.47 & 0.117 \\
\hline Hf-MOF-808/11 & Test & 7.37 & -5.76 & 13.13 & 1.65 & 0.068 \\
\hline Zr-NU-1000 (c pore)/14 & Test & -57.52 & -52.29 & -5.23 & -0.70 & 0.192 \\
\hline Zr-bi(cis)-defect UiO-66/11 & Test & -7.50 & -17.39 & 9.89 & 1.26 & 0.097 \\
\hline Zr-NU-1000 (c pore)/24 & Test & -57.90 & -45.96 & -11.94 & -1.55 & 0.125 \\
\hline Zr-MOF-808/14 & Test & 8.60 & 2.33 & 6.27 & 0.83 & 0.156 \\
\hline Hf-bi(trans)-defect UiO-66/6 & Test & -25.52 & -6.37 & -19.15 & -2.51 & 0.144 \\
\hline Zr-bi(trans)-defect UiO-66/18 & Test & -28.45 & -27.79 & -0.66 & -0.08 & 0.045 \\
\hline Th-MOF-808/13 & Test & -10.58 & -15.39 & 4.81 & 0.61 & 0.091 \\
\hline Th-bi(trans)-defect UiO-66/3 & Test & -37.58 & -35.31 & -2.27 & -0.29 & 0.096 \\
\hline Hf-MOF- $808 / 6$ & Test & 1.91 & -4.94 & 6.85 & 0.88 & 0.117 \\
\hline Zr-MOF-808/20 & Test & -24.90 & -19.36 & -5.54 & -0.72 & 0.127 \\
\hline Hf-MOF-808/ $\mathrm{H}_{2} \mathrm{O}$ & Test & -14.76 & -16.55 & 1.79 & 0.25 & 0.266 \\
\hline Ce-bi(trans)-defect UiO-66/22 & Test & -26.55 & -31.32 & 4.77 & 0.60 & 0.077 \\
\hline Zr-mono-defect UiO-66/14 & Test & -24.55 & -34.42 & 9.87 & 1.29 & 0.135 \\
\hline Zr-bi(trans)-defect UiO-66/11 & Test & -4.23 & -11.94 & 7.71 & 0.98 & 0.082 \\
\hline Ce-bi(trans)-defect UiO-66/18 & Test & -42.44 & -47.55 & 5.11 & 0.66 & 0.126 \\
\hline Ce-bi(trans)-defect UiO-66/1 & Test & -27.20 & -29.46 & 2.26 & 0.30 & 0.144 \\
\hline Th-MOF-808/6 & Test & -0.70 & -9.89 & 9.19 & 1.17 & 0.097 \\
\hline Th-MOF-808/1 & Test & -7.41 & -14.46 & 7.05 & 0.93 & 0.150 \\
\hline Hf-bi(trans)-defect UiO-66/14 & Test & -40.74 & -31.28 & -9.46 & -1.30 & 0.228 \\
\hline Zr-mono-defect UiO-66/1 & Test & -9.99 & -4.94 & -5.05 & -0.65 & 0.125 \\
\hline
\end{tabular}




\begin{tabular}{|c|c|c|c|c|c|c|}
\hline Th-bi(trans)-defect UiO-66/13 & Test & -36.50 & -29.08 & -7.42 & -0.95 & 0.099 \\
\hline Zr-MOF-808/1 & Test & -8.47 & -13.17 & 4.70 & 0.59 & 0.081 \\
\hline Zr-MOF-808/6 & Test & -4.81 & -7.08 & 2.27 & 0.28 & 0.062 \\
\hline Zr-bi(trans)-defect UiO-66/8 & Test & -12.14 & -16.96 & 4.82 & 0.60 & 0.042 \\
\hline Zr-bi(cis)-defect UiO-66/1 & Test & -22.57 & -16.23 & -6.34 & -0.80 & 0.074 \\
\hline Ce-MOF-808/24 & Test & -22.22 & -17.96 & -4.26 & -0.54 & 0.078 \\
\hline
\end{tabular}
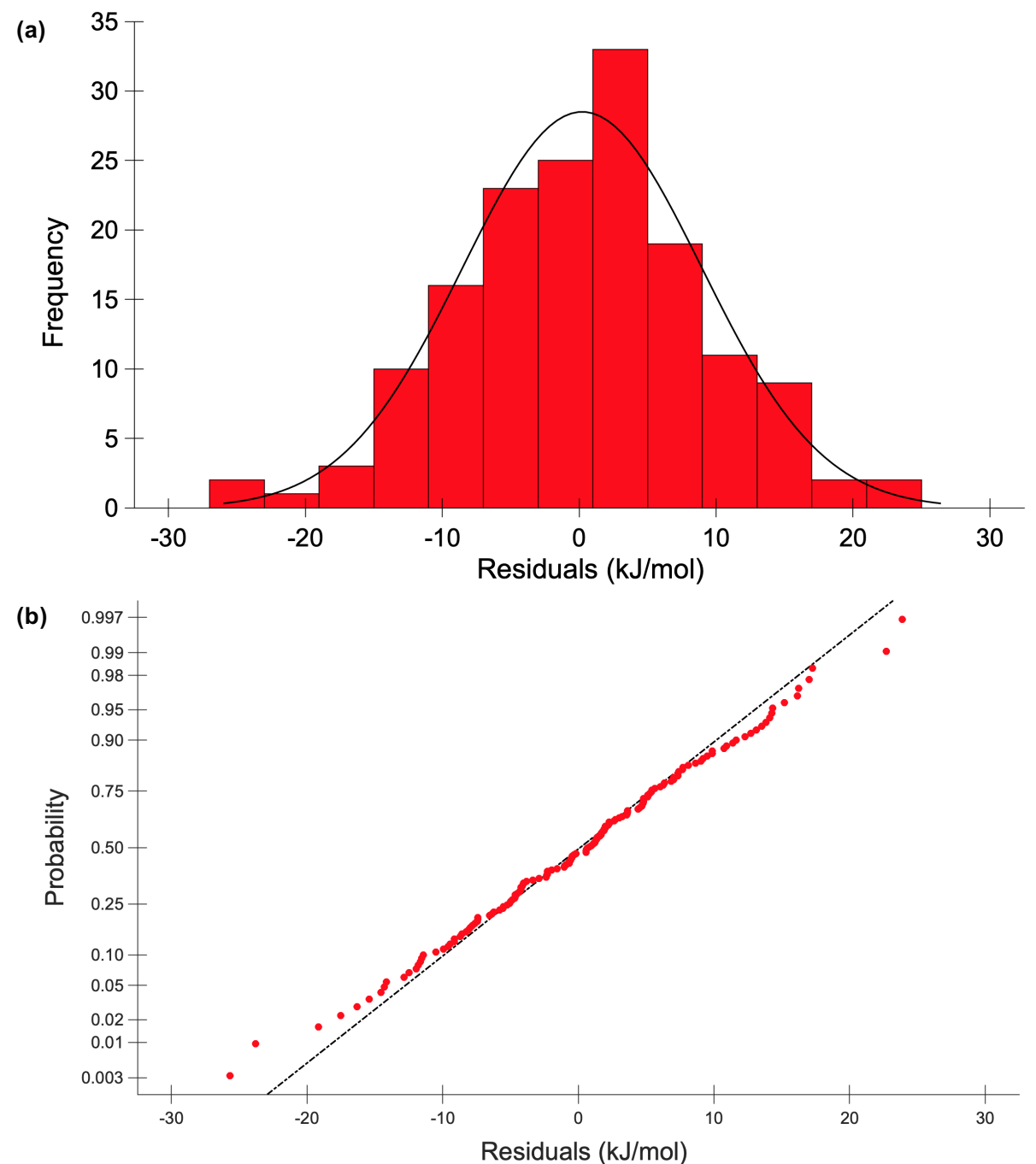

Figure S20. Distribution of residuals for the optimal predictive QSAR model developed for the neutrals dataset (eq 9 in the main text). The (a) histogram of raw residuals and (b) normal probability plot of raw residuals show that the residuals have an acceptable normal distribution, with no clear outliers. 


\section{QSAR Modeling: Optimal Predictive QSAR Model for Bidentates Dataset}

Table S17. Predictions, residuals, and leverages for training and test set molecule-node combinations using the optimal predictive QSAR model for the bidentates dataset (eq 10 in the main text).

\begin{tabular}{|c|c|c|c|c|c|c|}
\hline Node/Molecule & Set & $\begin{array}{c}\text { Observation } \\
(\mathbf{k J} / \mathbf{m o l})\end{array}$ & $\begin{array}{c}\text { Prediction } \\
(\mathrm{kJ} / \mathrm{mol})\end{array}$ & $\begin{array}{c}\text { Raw Residual } \\
(\mathrm{kJ} / \mathbf{m o l})\end{array}$ & $\begin{array}{c}\text { Standardized } \\
\text { Residual }\end{array}$ & Leverage \\
\hline Ce-bi(trans)-defect UiO-66/19 & Training & -157.38 & -146.68 & -10.70 & -1.03 & 0.149 \\
\hline Th-bi(trans)-defect UiO-66/12 & Training & -58.87 & -82.62 & 23.75 & 2.22 & 0.091 \\
\hline Zr-NU-1000 (large pore)/7 & Training & -92.74 & -90.77 & -1.97 & -0.18 & 0.028 \\
\hline Zr-bi(trans)-defect UiO-66/12 & Training & -70.69 & -88.84 & 18.15 & 1.67 & 0.067 \\
\hline Zr-NU-1000 (large pore)/15 & Training & -98.88 & -94.27 & -4.61 & -0.42 & 0.028 \\
\hline Zr-bi(trans)-defect UiO-66/7 & Training & -89.10 & -94.56 & 5.46 & 0.49 & 0.030 \\
\hline Hf-MOF-808/16 & Training & -94.97 & -82.54 & -12.43 & -1.18 & 0.125 \\
\hline Zr-NU-1000 (c pore)/9 & Training & -118.57 & -105.96 & -12.61 & -1.17 & 0.086 \\
\hline Zr-NU-1000 (c pore)/19 & Training & -138.38 & -120.43 & -17.95 & -1.69 & 0.106 \\
\hline Zr-NU-1000 (c pore)/16 & Training & -100.82 & -95.87 & -4.95 & -0.46 & 0.083 \\
\hline Th-bi(trans)-defect UiO-66/9 & Training & -92.55 & -97.01 & 4.46 & 0.42 & 0.096 \\
\hline Ce-MOF-808/7 & Training & -108.30 & -116.98 & 8.68 & 0.81 & 0.093 \\
\hline Hf-MOF-808/19 & Training & -118.91 & -107.10 & -11.81 & -1.15 & 0.158 \\
\hline Zr-NU-1000 (c pore)/12 & Training & -83.49 & -91.58 & 8.09 & 0.75 & 0.084 \\
\hline Ce-MOF-808/9 & Training & -120.02 & -125.64 & 5.62 & 0.54 & 0.125 \\
\hline Th-MOF-808/19 & Training & -119.16 & -104.86 & -14.30 & -1.37 & 0.141 \\
\hline Zr-mono-defect UiO-66/4 & Training & -85.40 & -99.60 & 14.20 & 1.32 & 0.077 \\
\hline Hf-bi(trans)-defect UiO-66/19 & Training & -109.98 & -113.41 & 3.43 & 0.33 & 0.136 \\
\hline Zr-NU-1000 (large pore)/12 & Training & -82.68 & -85.05 & 2.37 & 0.22 & 0.065 \\
\hline Zr-mono-defect UiO-66/23 & Training & -104.92 & -104.42 & -0.50 & -0.05 & 0.071 \\
\hline Hf-MOF-808/7 & Training & -90.80 & -83.98 & -6.82 & -0.64 & 0.091 \\
\hline Hf-bi(trans)-defect UiO-66/21 & Training & -95.16 & -107.02 & 11.86 & 1.11 & 0.088 \\
\hline Th-bi(trans)-defect UiO-66/16 & Training & -101.46 & -86.92 & -14.54 & -1.36 & 0.092 \\
\hline Zr-bi(trans)-defect UiO-66/21 & Training & -92.50 & -111.29 & 18.79 & 1.71 & 0.046 \\
\hline Th-MOF-808/7 & Training & -92.50 & -81.73 & -10.77 & -1.00 & 0.075 \\
\hline Zr-NU-1000 (c pore)/21 & Training & -119.30 & -114.03 & -5.27 & -0.48 & 0.062 \\
\hline Zr-NU-1000 (c pore)/23 & Training & -112.45 & -106.47 & -5.98 & -0.56 & 0.085 \\
\hline Th-bi(trans)-defect UiO-66/19 & Training & -87.40 & -111.47 & 24.07 & 2.28 & 0.116 \\
\hline Zr-bi(cis)-defect UiO-66/7 & Training & -96.01 & -95.21 & -0.80 & -0.07 & 0.033 \\
\hline Th-MOF-808/16 & Training & -95.33 & -80.30 & -15.03 & -1.42 & 0.110 \\
\hline Zr-MOF-808/9 & Training & -92.27 & -97.11 & 4.84 & 0.45 & 0.080 \\
\hline Zr-MOF-808/15 & Training & -101.41 & -91.94 & -9.47 & -0.86 & 0.045 \\
\hline Zr-bi(trans)-defect UiO-66/23 & Training & -101.57 & -103.73 & 2.16 & 0.20 & 0.068 \\
\hline
\end{tabular}




\begin{tabular}{|c|c|c|c|c|c|c|}
\hline Th-MOF-808/15 & Training & -99.97 & -85.23 & -14.74 & -1.37 & 0.077 \\
\hline Ce-MOF-808/21 & Training & -133.05 & -133.71 & 0.66 & 0.06 & 0.116 \\
\hline Ce-MOF-808/4 & Training & -112.07 & -121.32 & 9.25 & 0.88 & 0.128 \\
\hline Th-bi(trans)-defect UiO-66/4 & Training & -88.95 & -92.69 & 3.74 & 0.35 & 0.100 \\
\hline Zr-MOF-808/4 & Training & -77.99 & -92.79 & 14.80 & 1.38 & 0.086 \\
\hline Zr-mono-defect UiO-66/12 & Training & -100.97 & -89.53 & -11.44 & -1.06 & 0.070 \\
\hline Zr-mono-defect UiO-66/19 & Training & -108.18 & -118.38 & 10.20 & 0.95 & 0.093 \\
\hline Ce-bi(trans)-defect UiO-66/15 & Training & -130.05 & -127.05 & -3.00 & -0.28 & 0.089 \\
\hline Zr-MOF-808/23 & Training & -76.28 & -97.61 & 21.33 & 1.98 & 0.084 \\
\hline Ce-MOF-808/16 & Training & -107.23 & -115.55 & 8.32 & 0.79 & 0.129 \\
\hline Zr-mono-defect UiO-66/2 & Training & -97.92 & -101.42 & 3.50 & 0.32 & 0.029 \\
\hline Zr-MOF-808/2 & Training & -72.86 & -94.61 & 21.75 & 1.98 & 0.041 \\
\hline Zr-bi(cis)-defect UiO-66/21 & Training & -111.31 & -111.94 & 0.63 & 0.06 & 0.049 \\
\hline Zr-MOF-808/21 & Training & -98.84 & -105.18 & 6.34 & 0.58 & 0.064 \\
\hline Zr-bi(cis)-defect UiO-66/2 & Training & -115.40 & -101.37 & -14.03 & -1.27 & 0.029 \\
\hline Zr-NU-1000 (large pore)/9 & Training & -90.93 & -99.43 & 8.50 & 0.78 & 0.065 \\
\hline Zr-bi(trans)-defect UiO-66/19 & Training & -104.28 & -117.69 & 13.41 & 1.25 & 0.091 \\
\hline Th-bi(trans)-defect UiO-66/15 & Training & -101.63 & -91.85 & -9.78 & -0.90 & 0.057 \\
\hline Hf-bi(trans)-defect UiO-66/16 & Training & -96.51 & -88.85 & -7.66 & -0.72 & 0.108 \\
\hline Ce-bi(trans)-defect UiO-66/7 & Training & -131.37 & -123.55 & -7.82 & -0.73 & 0.086 \\
\hline Zr-mono-defect UiO-66/21 & Training & -109.14 & -111.98 & 2.84 & 0.26 & 0.049 \\
\hline Th-bi(trans)-defect UiO-66/21 & Training & -95.08 & -105.08 & 10.00 & 0.93 & 0.074 \\
\hline Th-bi(trans)-defect UiO-66/23 & Training & -83.96 & -97.52 & 13.56 & 1.27 & 0.097 \\
\hline Hf-MOF-808/9 & Training & -94.41 & -92.64 & -1.77 & -0.17 & 0.127 \\
\hline Zr-NU-1000 (large pore)/2 & Training & -102.57 & -96.94 & -5.63 & -0.51 & 0.025 \\
\hline Hf-MOF-808/2 & Training & -74.56 & -90.14 & 15.58 & 1.45 & 0.086 \\
\hline Zr-bi(cis)-defect UiO-66/12 & Training & -101.87 & -89.48 & -12.39 & -1.14 & 0.069 \\
\hline Ce-bi(trans)-defect UiO-66/12 & Training & -127.39 & -117.83 & -9.56 & -0.91 & 0.120 \\
\hline Zr-bi(cis)-defect UiO-66/15 & Training & -109.65 & -98.70 & -10.95 & -0.99 & 0.033 \\
\hline Hf-MOF-808/23 & Training & -78.99 & -93.15 & 14.16 & 1.35 & 0.124 \\
\hline Ce-bi(trans)-defect UiO-66/4 & Training & -129.87 & -127.89 & -1.98 & -0.19 & 0.124 \\
\hline Th-MOF-808/9 & Training & -106.03 & -90.39 & -15.64 & -1.48 & 0.113 \\
\hline Hf-bi(trans)-defect UiO-66/12 & Training & -74.95 & -84.56 & 9.61 & 0.91 & 0.116 \\
\hline Hf-bi(trans)-defect UiO-66/9 & Training & -110.57 & -98.95 & -11.62 & -1.10 & 0.112 \\
\hline Zr-mono-defect UiO-66/15 & Training & -89.83 & -98.75 & 8.92 & 0.81 & 0.033 \\
\hline Hf-bi(trans)-defect UiO-66/2 & Training & -103.62 & -96.46 & -7.16 & -0.66 & 0.068 \\
\hline Zr-NU-1000 (large pore)/4 & Training & -91.49 & -95.11 & 3.62 & 0.33 & 0.071 \\
\hline Zr-bi(trans)-defect UiO-66/2 & Training & -100.44 & -100.73 & 0.29 & 0.03 & 0.026 \\
\hline Zr-mono-defect UiO-66/9 & Training & -108.00 & -103.91 & -4.09 & -0.38 & 0.071 \\
\hline
\end{tabular}




\begin{tabular}{|c|c|c|c|c|c|c|}
\hline Ce-bi(trans)-defect UiO-66/9 & Training & -135.51 & -132.21 & -3.30 & -0.31 & 0.121 \\
\hline Th-MOF-808/12 & Training & -85.27 & -76.01 & -9.26 & -0.87 & 0.109 \\
\hline Ce-MOF-808/12 & Training & -114.96 & -111.25 & -3.71 & -0.35 & 0.126 \\
\hline Th-MOF-808/4 & Training & -95.47 & -86.08 & -9.39 & -0.89 & 0.117 \\
\hline Th-bi(trans)-defect UiO-66/7 & Training & -70.96 & -88.35 & 17.39 & 1.59 & 0.056 \\
\hline Zr-MOF-808/19 & Training & -119.41 & -111.57 & -7.84 & -0.74 & 0.110 \\
\hline Zr-MOF-808/16 & Training & -91.96 & -87.01 & -4.95 & -0.46 & 0.079 \\
\hline Zr-MOF-808/12 & Training & -83.00 & -82.72 & -0.28 & -0.03 & 0.080 \\
\hline Ce-bi(trans)-defect UiO-66/2 & Training & -135.32 & -129.72 & -5.60 & -0.52 & 0.084 \\
\hline Th-MOF-808/21 & Training & -113.47 & -98.46 & -15.01 & -1.41 & 0.096 \\
\hline Hf-bi(trans)-defect UiO-66/7 & Training & -92.40 & -90.29 & -2.11 & -0.20 & 0.075 \\
\hline Zr-NU-1000 (large pore)/21 & Training & -114.58 & -107.50 & -7.08 & -0.65 & 0.046 \\
\hline Ce-MOF-808/23 & Training & -105.88 & -126.15 & 20.27 & 1.94 & 0.138 \\
\hline Zr-mono-defect UiO-66/7 & Training & -82.91 & -95.25 & 12.34 & 1.12 & 0.033 \\
\hline Th-bi(trans)-defect UiO-66/2 & Training & -86.81 & -94.52 & 7.71 & 0.71 & 0.053 \\
\hline Zr-NU-1000 (c pore)/2 & Training & -111.63 & -103.47 & -8.16 & -0.74 & 0.043 \\
\hline Zr-mono-defect UiO-66/16 & Training & -75.76 & -93.82 & 18.06 & 1.67 & 0.068 \\
\hline Zr-bi(cis)-defect UiO-66/23 & Training & -121.62 & -104.37 & -17.25 & -1.59 & 0.071 \\
\hline Hf-bi(trans)-defect UiO-66/23 & Training & -105.05 & -99.46 & -5.59 & -0.53 & 0.106 \\
\hline Hf-bi(trans)-defect UiO-66/4 & Training & -99.71 & -94.63 & -5.08 & -0.48 & 0.122 \\
\hline Zr-NU-1000 (c pore)/4 & Training & -101.60 & -101.64 & 0.04 & 0.00 & 0.092 \\
\hline Zr-MOF-808/7 & Training & -87.76 & -88.45 & 0.69 & 0.06 & 0.044 \\
\hline Ce-bi(trans)-defect UiO-66/21 & Training & -154.70 & -140.28 & -14.42 & -1.36 & 0.106 \\
\hline Zr-bi(trans)-defect UiO-66/9 & Training & -107.89 & -103.22 & -4.67 & -0.43 & 0.068 \\
\hline Hf-MOF-808/21 & Test & -105.27 & -100.71 & -4.56 & -0.49 & 0.108 \\
\hline Zr-bi(trans)-defect UiO-66/4 & Test & -96.71 & -98.90 & 2.19 & 0.23 & 0.074 \\
\hline Zr-bi(cis)-defect UiO-66/16 & Test & -98.87 & -93.77 & -5.10 & -0.54 & 0.068 \\
\hline Ce-bi(trans)-defect UiO-66/16 & Test & -145.02 & -122.12 & -22.90 & -2.50 & 0.123 \\
\hline Zr-bi(cis)-defect UiO-66/19 & Test & -129.35 & -118.33 & -11.02 & -1.18 & 0.093 \\
\hline Zr-NU-1000 (large pore)/23 & Test & -90.24 & -99.94 & 9.70 & 1.03 & 0.067 \\
\hline Zr-bi(cis)-defect UiO-66/4 & Test & -100.00 & -99.55 & -0.45 & -0.05 & 0.077 \\
\hline Hf-MOF-808/4 & Test & -84.10 & -88.32 & 4.22 & 0.46 & 0.136 \\
\hline Ce-bi(trans)-defect UiO-66/23 & Test & -130.88 & -132.72 & 1.84 & 0.20 & 0.130 \\
\hline Zr-NU-1000 (large pore)/16 & Test & -102.89 & -89.34 & -13.55 & -1.43 & 0.063 \\
\hline Hf-MOF-808/15 & Test & -102.06 & -87.48 & -14.58 & -1.56 & 0.089 \\
\hline Zr-NU-1000 (large pore)/19 & Test & -122.33 & -113.90 & -8.43 & -0.91 & 0.092 \\
\hline Ce-MOF-808/15 & Test & -114.78 & -120.48 & 5.70 & 0.61 & 0.096 \\
\hline Zr-bi(trans)-defect UiO-66/16 & Test & -90.44 & -93.13 & 2.69 & 0.28 & 0.065 \\
\hline Hf-MOF-808/12 & Test & -80.79 & -78.25 & -2.54 & -0.28 & 0.132 \\
\hline
\end{tabular}




\begin{tabular}{|c|c|c|c|c|c|c|}
\hline Ce-MOF-808/19 & Test & -150.40 & -140.10 & -10.30 & -1.15 & 0.161 \\
\hline Hf-bi(trans)-defect UiO-66/15 & Test & -104.35 & -93.79 & -10.56 & -1.12 & 0.072 \\
\hline Zr-bi(trans)-defect UiO-66/15 & Test & -100.24 & -98.06 & -2.18 & -0.23 & 0.030 \\
\hline Zr-NU-1000 (c pore)/7 & Test & -102.89 & -97.30 & -5.59 & -0.59 & 0.047 \\
\hline Zr-bi(cis)-defect UiO-66/9 & Test & -119.69 & -103.87 & -15.82 & -1.68 & 0.071 \\
\hline Ce-MOF-808/2 & Test & -103.36 & -123.15 & 19.79 & 2.12 & 0.092 \\
\hline Th-MOF-808/23 & Test & -90.91 & -90.90 & -0.01 & 0.00 & 0.118 \\
\hline Zr-NU-1000 (c pore)/15 & Test & -109.16 & -100.80 & -8.36 & -0.88 & 0.047 \\
\hline Th-MOF-808/2 & Test & -87.97 & -87.90 & -0.07 & -0.01 & 0.073 \\
\hline
\end{tabular}
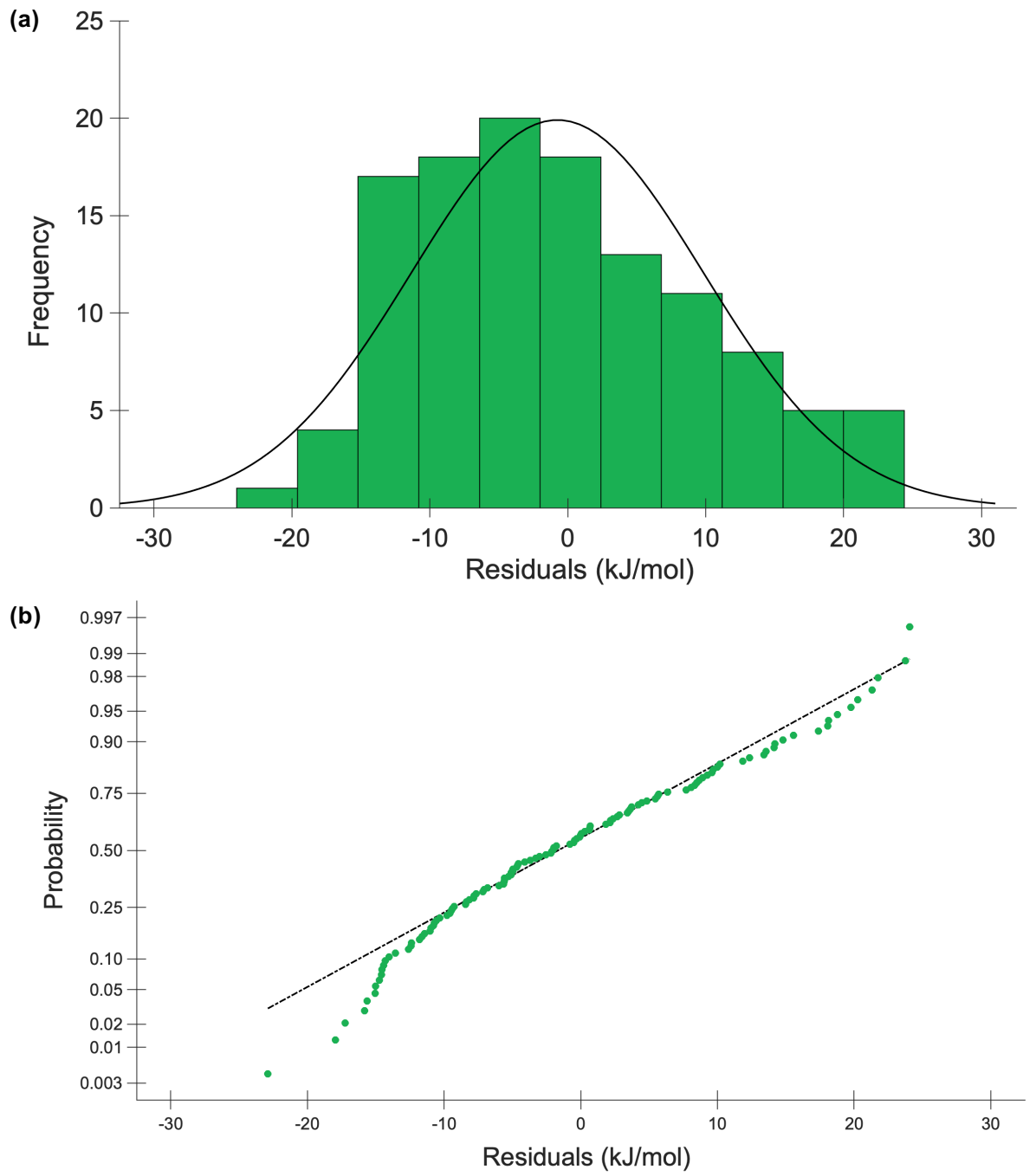

Figure S21. Distribution of residuals for the optimal predictive QSAR model developed for the bidentates dataset (eq 10 in the main text). The (a) histogram of raw residuals and (b) normal probability plot of raw residuals show that the residuals have an acceptable normal distribution, with no clear outliers. 


\section{Raw Energy Values for Individual Molecules}

In the tables below, the raw energy values are given for the optimized structures of all molecules explored as adsorbates in our study. These values correspond to the individual molecules (i.e., not including the nodes), where the free energies and enthalpies are computed (at $298.15 \mathrm{~K}$ and $1 \mathrm{~atm}$ ) by combining M06-2X(SMD)/def2-SVP single point energies with thermochemical contributions obtained at the M06-L(gas phase)/def2-SVP level of theory.

Table S18. The SCF energies, enthalpies, and free energies for the optimized structures of $\mathrm{HO}^{-}, \mathrm{H}_{2} \mathrm{O}$, and the 24 molecules shown in Figure 1a.

\begin{tabular}{|c|c|c|c|}
\hline Molecule & E (hartree) & H (hartree) & G (hartree) \\
\hline $\mathbf{H O}^{-}$ & -75.79112711 & -75.78782211 & -75.80739711 \\
\hline $\mathbf{H}_{\mathbf{2}}$ & -76.31370268 & -76.30992368 & -76.33135468 \\
\hline $\mathbf{1}$ & -797.652765 & -797.638929 & -797.692609 \\
\hline $\mathbf{2}$ & -780.2419274 & -780.2296934 & -780.2790564 \\
\hline $\mathbf{3}$ & -154.7658853 & -154.7607123 & -154.7911833 \\
\hline $\mathbf{4}$ & -701.7862968 & -701.7768758 & -701.8190038 \\
\hline $\mathbf{5}$ & -92.83313014 & -92.82982514 & -92.85218214 \\
\hline $\mathbf{6}$ & -749.435337 & -749.424162 & -749.471136 \\
\hline $\mathbf{7}$ & -724.9725816 & -724.9616666 & -725.0075376 \\
\hline $\mathbf{8}$ & -194.0071775 & -194.0008415 & -194.0344825 \\
\hline $\mathbf{9}$ & -607.27395 & -607.266904 & -607.30318 \\
\hline $\mathbf{1 0}$ & -99.8054447 & -99.8030837 & -99.8196027 \\
\hline $\mathbf{1 1}$ & -867.1442002 & -867.1290852 & -867.1845062 \\
\hline $\mathbf{1 2}$ & -842.6827081 & -842.6678621 & -842.7222021 \\
\hline $\mathbf{1 3}$ & -311.7154039 & -311.7051949 & -311.7479319 \\
\hline $\mathbf{1 4}$ & -1378.213728 & -1378.191399 & -1378.263557 \\
\hline $\mathbf{1 5}$ & -685.7305245 & -685.7207995 & -685.7644565 \\
\hline $\mathbf{1 6}$ & -1299.30352 & -1299.284602 & -1299.348555 \\
\hline $\mathbf{1 7}$ & -768.3469004 & -768.3327824 & -768.3858964 \\
\hline $\mathbf{1 8}$ & -901.3676632 & -901.3516812 & -901.4097862 \\
\hline $\mathbf{1 9}$ & -876.8884184 & -876.8723754 & -876.9306284 \\
\hline $\mathbf{2 0}$ & -976.5125825 & -976.4953785 & -976.5570135 \\
\hline $\mathbf{2 1}$ & -952.0425965 & -952.0256145 & -952.0865275 \\
\hline $\mathbf{2 2}$ & -686.1609106 & -686.1502166 & -686.1957616 \\
\hline $\mathbf{2 3}$ & -646.4909169 & -646.4823659 & -646.5227419 \\
\hline $\mathbf{2 4}$ & -115.5262468 & -115.5220108 & -115.5489478 \\
\hline & & & \\
\hline & & & \\
\hline
\end{tabular}




\section{Raw Energy Values for All Molecules Bound to Each M(IV)-MOF Node Site}

In the tables below, the raw energy values are given for the optimized structures of all molecules bound to each of the 12 different M(IV)-MOF node sites: Zr-mono-defect UiO-66, M-bi(trans)defect UiO-66 (M = Zr, Hf, Ce, Th), Zr-bi(cis)-defect UiO-66, Zr-NU-1000 (large pore), Zr-NU1000 (c pore), and M-MOF-808 (M = Zr, Hf, Ce, Th). The free energies and enthalpies are computed (at 298.15 K and $1 \mathrm{~atm}$ ) by combining M06-2X(SMD)/def2-SVP single point energies with thermochemical contributions obtained at the M06-L(gas phase)/def2-SVP level of theory. All systems labeled node correspond to the original, fully hydrated state of the node site. The nodenoOH $\mathrm{H}_{2}$ structures represent a node where the terminal $-\mathrm{OH}_{2}$ group has been removed from the active (binding) site, the node-noOH structures represent a node where the terminal $-\mathrm{OH}$ group has been removed from the binding site, and the node-noOH $\mathrm{OH}_{2} \mathrm{OH}$ structures represent a node where the terminal $-\mathrm{OH}_{2}$ and $-\mathrm{OH}$ groups have been removed from the binding site. The node- $\mathbf{N}$ structures represent molecule $\mathbf{N}$ (i.e., one of the 24 molecules shown in Figure 1a) bound to the node site. For each of the different node sites, the energies are only provided for the molecules bound to the node site in their most favorable orientations (identified in Tables S1 and S2). 
Table S19. The SCF energies, enthalpies, and free energies for the optimized structures of the bare node of Zr-mono-defect UiO-66 and different node-bound molecules.

\begin{tabular}{|c|c|c|c|}
\hline System & E (hartree) & H (hartree) & G (hartree) \\
\hline node & -5656.118631 & -5656.065287 & -5656.190836 \\
\hline node-noOH ${ }_{2}$ & -5579.775234 & -5579.723336 & -5579.846676 \\
\hline node-noOH & -5580.225261 & -5580.172491 & -5580.29798 \\
\hline node-noOH ${ }_{2}, \mathrm{OH}$ & -5503.906621 & -5503.856369 & -5503.976821 \\
\hline node-1 & -6377.452956 & -6377.386835 & -6377.540304 \\
\hline node-2 & -6284.210966 & -6284.149129 & -6284.293173 \\
\hline node- $\mathbf{3}$ & -5734.564846 & -5734.50881 & -5734.640166 \\
\hline node-4 & -6205.749112 & -6205.689872 & -6205.828351 \\
\hline node $-\mathbf{5}$ & -5673.088678 & -5673.033906 & -5673.162976 \\
\hline node-6 & -6329.23646 & -6329.173194 & -6329.319804 \\
\hline node -7 & -6228.935363 & -6228.874714 & -6229.015936 \\
\hline node $-\mathbf{8}$ & -5773.802745 & -5773.745156 & -5773.879975 \\
\hline node $-\mathbf{9}$ & -6111.245344 & -6111.188839 & -6111.321137 \\
\hline node $-\mathbf{1 0}$ & -5680.142627 & -5680.089557 & -5680.214704 \\
\hline node-11 & -6446.945639 & -6446.878543 & -6447.032526 \\
\hline node-12 & -6346.653711 & -6346.589343 & -6346.737479 \\
\hline node $-\mathbf{1 3}$ & -5891.52023 & -5891.458719 & -5891.601045 \\
\hline node-14 & -6958.020998 & -6957.946074 & -6958.116798 \\
\hline node-15 & -6189.696117 & -6189.636919 & -6189.775492 \\
\hline node-16 & -6803.265694 & -6803.196957 & -6803.354231 \\
\hline node $-\mathbf{1 7}$ & -6348.131284 & -6348.064939 & -6348.217924 \\
\hline node-18 & -6481.174902 & -6481.107348 & -6481.262211 \\
\hline node $-\mathbf{1 9}$ & -6380.863223 & -6380.797922 & -6380.948654 \\
\hline node-20 & -6556.327736 & -6556.258784 & -6556.417022 \\
\hline node-21 & -6456.017599 & -6455.95101 & -6456.104919 \\
\hline node-22 & -6265.956934 & -6265.894321 & -6266.040057 \\
\hline node-23 & -6150.461729 & -6150.40378 & -6150.539523 \\
\hline node-24 & -5695.327009 & -5695.271934 & -5695.401546 \\
\hline
\end{tabular}


Table S20. The SCF energies, enthalpies, and free energies for the optimized structures of the bare node of Zr-bi(trans)-defect UiO-66 and different node-bound molecules.

\begin{tabular}{|c|c|c|c|}
\hline System & E (hartree) & H (hartree) & G (hartree) \\
\hline node & -5388.477945 & -5388.423571 & -5388.551232 \\
\hline node-noOH ${ }_{2}$ & -5312.135774 & -5312.082714 & -5312.208478 \\
\hline node-noOH & -5312.590178 & -5312.536305 & -5312.664121 \\
\hline node-noOH ${ }_{2}, \mathrm{OH}$ & -5236.267579 & -5236.21616 & $\begin{array}{l}-5236.339106 \\
\end{array}$ \\
\hline node-1 & -6109.811587 & -6109.744704 & -6109.899338 \\
\hline node $-\mathbf{2}$ & -6016.574072 & -6016.511501 & -6016.656419 \\
\hline node $-\mathbf{3}$ & -5466.930252 & -5466.872712 & -5467.007813 \\
\hline node-4 & -5938.114637 & -5938.054144 & -5938.194943 \\
\hline node $-\mathbf{5}$ & -5405.450806 & -5405.394877 & -5405.52637 \\
\hline node-6 & -6061.601913 & -6061.537552 & -6061.686092 \\
\hline node -7 & -5961.299197 & -5961.237569 & -5961.380582 \\
\hline node $-\mathbf{8}$ & -5506.168977 & -5506.110081 & -5506.247585 \\
\hline node -9 & -5843.606173 & -5843.548411 & -5843.683381 \\
\hline node-10 & -5412.502704 & -5412.448552 & -5412.575955 \\
\hline node-11 & -6179.306729 & -6179.23838 & -6179.394597 \\
\hline node-12 & -6079.003847 & -6078.9385 & -6079.088232 \\
\hline node-13 & -5623.879154 & -5623.816423 & -5623.961288 \\
\hline node-14 & -6690.391266 & -6690.315691 & -6690.486928 \\
\hline node-15 & -5922.061104 & -5922.000753 & -5922.141744 \\
\hline node-16 & -6535.633382 & -6535.564067 & -6535.722108 \\
\hline node-17 & -6080.487522 & -6080.420724 & -6080.573978 \\
\hline node- $\mathbf{1 8}$ & -6213.539285 & -6213.470038 & -6213.629102 \\
\hline node-19 & -6113.222604 & -6113.156096 & -6113.309452 \\
\hline node-20 & -6288.688778 & -6288.618815 & -6288.778803 \\
\hline node-21 & -6188.373381 & -6188.305947 & -6188.460864 \\
\hline node-22 & -5998.322386 & -5998.25891 & -5998.40592 \\
\hline node-23 & -5882.821383 & -5882.762246 & -5882.900532 \\
\hline node-24 & -5427.690141 & -5427.633866 & -5427.766086 \\
\hline
\end{tabular}


Table S21. The SCF energies, enthalpies, and free energies for the optimized structures of the bare node of Hf-bi(trans)-defect UiO-66 and different node-bound molecules.

\begin{tabular}{|c|c|c|c|}
\hline System & E (hartree) & H (hartree) & G (hartree) \\
\hline node & -5394.398786 & -5394.343246 & -5394.475277 \\
\hline node-noOH & -5318.054584 & -5318.000429 & -5318.130452 \\
\hline node-noOH & -5318.511663 & -5318.456787 & -5318.588547 \\
\hline node-noOH ${ }_{2}, \mathrm{OH}$ & -5242.187842 & -5242.135473 & -5242.262347 \\
\hline node-1 & -6115.734507 & -6115.666768 & -6115.824797 \\
\hline node $-\mathbf{2}$ & -6022.495436 & -6022.431742 & -6022.580868 \\
\hline node $-\mathbf{3}$ & -5472.852264 & -5472.793702 & -5472.932866 \\
\hline node-4 & -5944.035951 & -5943.974392 & -5944.119328 \\
\hline node $-\mathbf{5}$ & -5411.37222 & -5411.315227 & -5411.450849 \\
\hline node-6 & -6067.524058 & -6067.458614 & -6067.611307 \\
\hline node -7 & -5967.220796 & -5967.158187 & -5967.305077 \\
\hline node $-\mathbf{8}$ & -5512.090142 & -5512.030313 & -5512.171628 \\
\hline node -9 & -5849.52749 & -5849.468718 & -5849.60764 \\
\hline node-10 & -5418.424251 & -5418.369012 & -5418.500611 \\
\hline node-11 & -6185.229139 & -6185.159829 & -6185.319968 \\
\hline node-12 & -6084.925741 & -6084.8594 & -6085.013097 \\
\hline node-13 & -5629.806284 & -5629.742637 & -5629.891141 \\
\hline node-14 & -6696.310871 & -6696.234331 & -6696.409526 \\
\hline node-15 & -5927.982672 & -5927.921155 & -5928.066548 \\
\hline node-16 & -6541.555926 & -6541.485556 & -6541.647661 \\
\hline node-17 & -6086.410767 & -6086.342957 & -6086.500234 \\
\hline node- $\mathbf{1 8}$ & -6219.460514 & -6219.390426 & -6219.552992 \\
\hline node-19 & -6119.145193 & -6119.077734 & -6119.234864 \\
\hline node-20 & -6294.610581 & -6294.539775 & -6294.703133 \\
\hline node-21 & -6194.294685 & -6194.226203 & -6194.38512 \\
\hline node-22 & -6004.243922 & -6004.179535 & -6004.330222 \\
\hline node-23 & -5888.742843 & -5888.682561 & -5888.825102 \\
\hline node-24 & -5433.611543 & -5433.554216 & -5433.690552 \\
\hline
\end{tabular}


Table S22. The SCF energies, enthalpies, and free energies for the optimized structures of the bare node of Ce-bi(trans)-defect UiO-66 and different node-bound molecules.

\begin{tabular}{|c|c|c|c|}
\hline System & E (hartree) & H (hartree) & G (hartree) \\
\hline node & -7955.73648 & -7955.672509 & -7955.824021 \\
\hline node-noOH & -7879.394036 & -7879.33191 & -7879.480488 \\
\hline node-noOH & -7879.847851 & -7879.784932 & -7879.935577 \\
\hline node-noOH ${ }_{2}, \mathrm{OH}$ & -7803.515815 & -7803.455904 & -7803.600018 \\
\hline node-1 & -8677.082259 & -8677.006307 & -8677.183458 \\
\hline node $-\mathbf{2}$ & -8583.834711 & -8583.763142 & -8583.930615 \\
\hline node $-\mathbf{3}$ & -8034.192059 & -8034.125284 & -8034.283327 \\
\hline node-4 & -8505.374251 & -8505.304634 & -8505.468488 \\
\hline node $-\mathbf{5}$ & -7972.70845 & -7972.643211 & -7972.79834 \\
\hline node-6 & -8628.865809 & -8628.792453 & -8628.963486 \\
\hline node -7 & -8528.562458 & -8528.491732 & -8528.657592 \\
\hline node $-\mathbf{8}$ & -8073.432926 & -8073.364807 & -8073.525449 \\
\hline node -9 & -8410.863842 & -8410.797 & -8410.95481 \\
\hline node-10 & -7979.763039 & -7979.699451 & -7979.850433 \\
\hline node-11 & -8746.575413 & -8746.498176 & -8746.676494 \\
\hline node-12 & -8646.272415 & -8646.197916 & -8646.370739 \\
\hline node-13 & -8191.143926 & -8191.071968 & -8191.239968 \\
\hline node-14 & -9257.6567 & -9257.571755 & -9257.766843 \\
\hline node-15 & -8489.319744 & -8489.250343 & -8489.41401 \\
\hline node-16 & -9102.901713 & -9102.823488 & -9103.003808 \\
\hline node-17 & -8647.764331 & -8647.687999 & -8647.86528 \\
\hline node- $\mathbf{1 8}$ & -8780.80301 & -8780.724646 & -8780.90644 \\
\hline node-19 & -8680.490528 & -8680.415141 & -8680.59059 \\
\hline node-20 & -8855.95238 & -8855.873531 & -8856.055791 \\
\hline node-21 & -8755.644481 & -8755.567936 & -8755.745466 \\
\hline node-22 & -8565.588904 & -8565.516324 & -8565.686362 \\
\hline node-23 & -8450.079651 & -8450.011404 & -8450.172608 \\
\hline node-24 & -7994.951199 & -7994.885888 & -7995.040436 \\
\hline
\end{tabular}


Table S23. The SCF energies, enthalpies, and free energies for the optimized structures of the bare node of Th-bi(trans)-defect UiO-66 and different node-bound molecules.

\begin{tabular}{|c|c|c|c|}
\hline System & E (hartree) & H (hartree) & G (hartree) \\
\hline node & -7552.466932 & -7552.401992 & -7552.558323 \\
\hline node-noOH ${ }_{2}$ & -7476.123089 & -7476.060376 & -7476.212413 \\
\hline node-noOH & -7476.588923 & -7476.525581 & -7476.679351 \\
\hline node-noOH ${ }_{2}, \mathrm{OH}$ & -7400.271212 & -7400.211109 & -7400.357696 \\
\hline node-1 & -8273.808234 & -8273.73182 & -8273.912252 \\
\hline node $-\mathbf{2}$ & -8180.570303 & -8180.497943 & -8180.669815 \\
\hline node $-\mathbf{3}$ & -7630.922902 & -7630.855235 & -7631.017911 \\
\hline node-4 & -8102.113165 & -8102.042872 & -8102.21058 \\
\hline node $-\mathbf{5}$ & -7569.446658 & -7569.380912 & -7569.539552 \\
\hline node-6 & -8225.586529 & -8225.512132 & -8225.688371 \\
\hline node -7 & -8125.294321 & -8125.223043 & -8125.39226 \\
\hline node $-\mathbf{8}$ & -7670.164226 & -7670.095393 & -7670.260049 \\
\hline node -9 & -8007.602309 & -8007.534932 & -8007.696125 \\
\hline node-10 & -7576.494968 & -7576.430531 & -7576.586216 \\
\hline node-11 & -8343.298559 & -8343.220079 & -8343.404285 \\
\hline node-12 & -8243.001264 & -8242.926244 & -8243.102321 \\
\hline node-13 & -7787.875339 & -7787.802743 & -7787.974246 \\
\hline node-14 & -8854.380397 & -8854.294962 & -8854.493458 \\
\hline node-15 & -8086.063346 & -8085.993251 & -8086.16086 \\
\hline node-16 & -8699.638717 & -8699.559546 & -8699.744896 \\
\hline node-17 & -8244.481631 & -8244.404753 & -8244.585385 \\
\hline node- $\mathbf{1 8}$ & -8377.526902 & -8377.448046 & -8377.633189 \\
\hline node-19 & -8277.217714 & -8277.14136 & -8277.321613 \\
\hline node-20 & -8452.674338 & -8452.594519 & -8452.781799 \\
\hline node-21 & -8352.376286 & -8352.299183 & -8352.480437 \\
\hline node-22 & -8162.31298 & -8162.239324 & -8162.414808 \\
\hline node-23 & -8046.816452 & -8046.747589 & -8046.912417 \\
\hline node-24 & -7591.690973 & -7591.624636 & -7591.784272 \\
\hline
\end{tabular}


Table S24. The SCF energies, enthalpies, and free energies for the optimized structures of the bare node of Zr-bi(cis)-defect UiO-66 and different node-bound molecules.

\begin{tabular}{|c|c|c|c|}
\hline System & E (hartree) & H (hartree) & G (hartree) \\
\hline node & -5388.483536 & -5388.429339 & -5388.556655 \\
\hline node-noOH ${ }_{2}$ & -5312.131629 & -5312.078447 & -5312.204557 \\
\hline node-noOH & -5312.587401 & -5312.533589 & -5312.661022 \\
\hline node-noOH ${ }_{2}, \mathrm{OH}$ & -5236.259624 & -5236.208195 & -5236.331194 \\
\hline node-1 & -6109.818544 & -6109.751812 & -6109.905763 \\
\hline node-2 & -6016.570295 & -6016.507123 & -6016.654205 \\
\hline node- $\mathbf{3}$ & -5466.92403 & -5466.866722 & -5467.000918 \\
\hline node-4 & -5938.108068 & -5938.047732 & -5938.188284 \\
\hline node $-\mathbf{5}$ & -5405.442714 & -5405.38685 & -5405.518333 \\
\hline node-6 & -6061.599084 & -6061.53489 & -6061.683421 \\
\hline node -7 & -5961.294202 & -5961.232725 & -5961.3753 \\
\hline node $-\mathbf{8}$ & -5506.167217 & -5506.108344 & -5506.245503 \\
\hline node $-\mathbf{9}$ & -5843.603224 & -5843.545865 & -5843.67996 \\
\hline node $-\mathbf{1 0}$ & -5412.509281 & -5412.455331 & -5412.582313 \\
\hline node-11 & -6179.303484 & -6179.235154 & -6179.39192 \\
\hline node-12 & -6079.008343 & -6078.943343 & -6079.092196 \\
\hline node $-\mathbf{1 3}$ & -5623.882704 & -5623.820215 & -5623.964337 \\
\hline node-14 & -6690.378224 & -6690.30223 & -6690.475518 \\
\hline node-15 & -5922.057501 & -5921.997203 & -5922.137413 \\
\hline node-16 & -6535.627161 & -6535.557096 & -6535.717405 \\
\hline node $-\mathbf{1 7}$ & -6080.485575 & -6080.418574 & -6080.572631 \\
\hline node-18 & -6213.542952 & -6213.47406 & -6213.631777 \\
\hline node $-\mathbf{1 9}$ & -6113.225023 & -6113.158652 & -6113.311088 \\
\hline node-20 & -6288.678405 & -6288.607942 & -6288.769981 \\
\hline node-21 & -6188.372727 & -6188.305828 & -6188.460118 \\
\hline node-22 & -5998.325746 & -5998.262194 & -5998.409529 \\
\hline node-23 & -5882.821044 & -5882.761866 & -5882.900256 \\
\hline node-24 & -5427.69175 & -5427.635597 & -5427.767369 \\
\hline
\end{tabular}


Table S25. The SCF energies, enthalpies, and free energies for the optimized structures of the bare node of Zr-NU-1000 (large pore) and different node-bound molecules.

\begin{tabular}{|c|c|c|c|}
\hline System & E (hartree) & H (hartree) & G (hartree) \\
\hline node & -4853.196352 & -4853.139433 & -4853.272827 \\
\hline node-noOH ${ }_{2}$ & -4776.854843 & -4776.799192 & -4776.930784 \\
\hline node-noOH & -4777.299605 & -4777.243209 & -4777.376568 \\
\hline node-noOH ${ }_{2}, \mathrm{OH}$ & -4700.982002 & -4700.92818 & -4701.05657 \\
\hline node-1 & -5574.531611 & -5574.462159 & -5574.622683 \\
\hline node-2 & -5481.289338 & -5481.224249 & -5481.374693 \\
\hline node $-\mathbf{3}$ & -4931.645395 & -4931.58533 & -4931.726082 \\
\hline node -4 & -5402.827427 & -5402.764641 & -5402.910421 \\
\hline node $-\mathbf{5}$ & -4870.167379 & -4870.10901 & -4870.246013 \\
\hline node-6 & -5526.315657 & -5526.249072 & -5526.402938 \\
\hline node -7 & -5426.014696 & -5425.950519 & -5426.099432 \\
\hline node $-\mathbf{8}$ & -4970.885211 & -4970.824136 & -4970.966321 \\
\hline node -9 & -5308.314622 & -5308.254743 & -5308.394383 \\
\hline node-10 & -4877.221318 & -4877.164591 & -4877.297807 \\
\hline node-11 & -5644.025169 & -5643.95437 & -5644.116349 \\
\hline node-12 & -5543.721891 & -5543.65377 & -5543.810264 \\
\hline node-13 & -5088.600362 & -5088.535219 & -5088.685387 \\
\hline node-14 & -6155.107526 & -6155.029711 & -6155.205972 \\
\hline node-15 & -5386.775737 & -5386.712971 & -5386.858688 \\
\hline node-16 & -6000.352909 & -6000.281306 & -6000.444313 \\
\hline node-17 & -5545.207209 & -5545.137873 & -5545.296833 \\
\hline node- $\mathbf{1 8}$ & -5678.25615 & -5678.184853 & -5678.347675 \\
\hline node-19 & -5577.944821 & -5577.876011 & -5578.033793 \\
\hline node-20 & -5753.404594 & -5753.332458 & -5753.497752 \\
\hline node-21 & -5653.096091 & -5653.026229 & -5653.18674 \\
\hline node-22 & -5463.040913 & -5462.974693 & -5463.128452 \\
\hline node-23 & -5347.531729 & -5347.470262 & -5347.613684 \\
\hline node-24 & -4892.396699 & -4892.338046 & -4892.475726 \\
\hline
\end{tabular}


Table S26. The SCF energies, enthalpies, and free energies for the optimized structures of the bare node of Zr-NU-1000 (c pore) and different node-bound molecules.

\begin{tabular}{|c|c|c|c|}
\hline System & E (hartree) & H (hartree) & G (hartree) \\
\hline node & -4853.196352 & -4853.139433 & -4853.272827 \\
\hline node-noOH ${ }_{2}$ & -4776.842322 & -4776.786737 & -4776.918207 \\
\hline node-noOH & -4777.306331 & -4777.249809 & -4777.383596 \\
\hline node-noOH ${ }_{2}, \mathrm{OH}$ & -4700.980849 & -4700.926952 & -4701.05561 \\
\hline node $-\mathbf{1}$ & -5574.531241 & -5574.461608 & -5574.62234 \\
\hline node-2 & -5481.29208 & -5481.227105 & -5481.377185 \\
\hline node $-\mathbf{3}$ & -4931.651625 & -4931.5919 & -4931.731349 \\
\hline node-4 & -5402.830526 & -5402.767827 & -5402.91331 \\
\hline node $-\mathbf{5}$ & -4870.169405 & -4870.111052 & -4870.248046 \\
\hline node-6 & -5526.322265 & -5526.25554 & -5526.409642 \\
\hline node -7 & -5426.018559 & -5425.954684 & -5426.102337 \\
\hline node $-\mathbf{8}$ & -4970.881854 & -4970.820717 & -4970.963222 \\
\hline node -9 & -5308.324475 & -5308.264793 & -5308.403951 \\
\hline node-10 & -4877.221576 & -4877.164893 & -4877.297998 \\
\hline node-11 & -5644.027928 & -5643.957471 & -5644.118187 \\
\hline node-12 & -5543.721856 & -5543.65394 & -5543.809614 \\
\hline node-13 & -5088.586975 & -5088.521956 & -5088.671879 \\
\hline node-14 & -6155.105182 & -6155.027333 & -6155.203672 \\
\hline node-15 & -5386.778775 & -5386.716078 & -5386.861644 \\
\hline node-16 & -6000.351285 & -6000.279666 & -6000.442567 \\
\hline node-17 & -5545.21498 & -5545.14547 & -5545.304848 \\
\hline node-18 & -5678.259782 & -5678.188626 & -5678.351696 \\
\hline node-19 & -5577.949155 & -5577.8802 & -5578.038945 \\
\hline node-20 & -5753.404617 & -5753.331962 & -5753.498518 \\
\hline node-21 & -5653.097009 & -5653.027133 & -5653.187577 \\
\hline node-22 & -5463.044204 & -5462.977966 & -5463.131248 \\
\hline node-23 & -5347.539225 & -5347.477694 & -5347.621182 \\
\hline node-24 & -4892.410409 & -4892.351654 & -4892.48921 \\
\hline
\end{tabular}


Table S27. The SCF energies, enthalpies, and free energies for the optimized structures of the bare node of Zr-MOF-808 and different node-bound molecules.

\begin{tabular}{|c|c|c|c|}
\hline System & E (hartree) & H (hartree) & G (hartree) \\
\hline node & -4317.898628 & -4317.839264 & -4317.977487 \\
\hline node-noOH ${ }_{2}$ & -4241.564524 & -4241.506279 & -4241.643119 \\
\hline node-noOH & -4242.008306 & -4241.949483 & -4242.087701 \\
\hline node-noOH ${ }_{2}, \mathrm{OH}$ & -4165.687525 & -4165.630919 & -4165.765186 \\
\hline node-1 & -5039.245446 & -5039.173586 & -5039.338956 \\
\hline node-2 & -4945.982887 & -4945.914774 & -4946.071994 \\
\hline node $-\mathbf{3}$ & -4396.347257 & -4396.284839 & -4396.430208 \\
\hline node-4 & -4867.527467 & -4867.461936 & -4867.613895 \\
\hline node $-\mathbf{5}$ & -4334.870354 & -4334.809538 & -4334.951568 \\
\hline node-6 & -4991.0261 & -4990.956995 & -4991.116088 \\
\hline node -7 & -4890.719235 & -4890.652455 & -4890.80615 \\
\hline node $-\mathbf{8}$ & -4435.59668 & -4435.533279 & -4435.680019 \\
\hline node -9 & -4773.021495 & -4772.959324 & -4773.103508 \\
\hline node-10 & -4341.920417 & -4341.861365 & -4341.9992 \\
\hline node-11 & -5108.734803 & -5108.66151 & -5108.828452 \\
\hline node-12 & -5008.428551 & -5008.357959 & -5008.519 \\
\hline node-13 & -4553.297442 & -4553.229859 & -4553.38499 \\
\hline node-14 & -5619.801587 & -5619.721228 & -5619.903402 \\
\hline node -15 & -4851.482635 & -4851.417332 & -4851.568266 \\
\hline node-16 & -5465.053326 & -5464.97864 & -5465.148767 \\
\hline node-17 & -5009.922307 & -5009.849487 & -5010.016679 \\
\hline node-18 & -5142.965416 & -5142.89186 & -5143.059581 \\
\hline node-19 & -5042.648976 & -5042.577338 & -5042.741294 \\
\hline node-20 & -5218.114099 & -5218.039485 & -5218.209617 \\
\hline node-21 & -5117.795292 & -5117.722781 & -5117.889358 \\
\hline node-22 & -4927.751816 & -4927.683451 & -4927.840909 \\
\hline node-23 & -4812.233157 & -4812.169336 & -4812.316982 \\
\hline node-24 & -4357.119734 & -4357.058649 & -4357.201043 \\
\hline
\end{tabular}


Table S28. The SCF energies, enthalpies, and free energies for the optimized structures of the bare node of Hf-MOF-808 and different node-bound molecules.

\begin{tabular}{|c|c|c|c|}
\hline System & E (hartree) & H (hartree) & G (hartree) \\
\hline node & -4323.829531 & -4323.769238 & -4323.911458 \\
\hline node-noOH ${ }_{2}$ & -4247.492686 & -4247.433458 & -4247.574481 \\
\hline node-noOH & -4247.93342 & -4247.873766 & -4248.015796 \\
\hline node-noOH ${ }_{2}, \mathrm{OH}$ & -4171.614109 & -4171.556501 & -4171.694822 \\
\hline node-1 & -5045.170632 & -5045.097767 & -5045.267242 \\
\hline node-2 & -4951.910006 & -4951.840823 & -4952.002278 \\
\hline node $-\mathbf{3}$ & -4402.272459 & -4402.208862 & -4402.359038 \\
\hline node-4 & -4873.457082 & -4873.391035 & -4873.545859 \\
\hline node $-\mathbf{5}$ & -4340.796968 & -4340.735214 & -4340.881166 \\
\hline node-6 & -4996.951962 & -4996.881878 & -4997.04489 \\
\hline node -7 & -4896.647094 & -4896.579343 & -4896.736943 \\
\hline node $-\mathbf{8}$ & -4441.524555 & -4441.460008 & -4441.611159 \\
\hline node -9 & -4778.948835 & -4778.885605 & -4779.03396 \\
\hline node-10 & -4347.853614 & -4347.793489 & -4347.935621 \\
\hline node-11 & -5114.659774 & -5114.585651 & -5114.75618 \\
\hline node-12 & -5014.354238 & -5014.282559 & -5014.447796 \\
\hline node-13 & -4559.224601 & -4559.155993 & -4559.315281 \\
\hline node-14 & -5625.727632 & -5625.646235 & -5625.832626 \\
\hline node -15 & -4857.409434 & -4857.343147 & -4857.49815 \\
\hline node-16 & -5470.980853 & -5470.904993 & -5471.079547 \\
\hline node-17 & -5015.849825 & -5015.776639 & -5015.945947 \\
\hline node-18 & -5148.891684 & -5148.817159 & -5148.988823 \\
\hline node-19 & -5048.575591 & -5048.50299 & -5048.670743 \\
\hline node-20 & -5224.040547 & -5223.964873 & -5224.139126 \\
\hline node-21 & -5123.724225 & -5123.650452 & -5123.821446 \\
\hline node-22 & -4933.679513 & -4933.609891 & -4933.772234 \\
\hline node-23 & -4818.160389 & -4818.095414 & -4818.247648 \\
\hline node-24 & -4363.045055 & -4362.982869 & -4363.129657 \\
\hline
\end{tabular}


Table S29. The SCF energies, enthalpies, and free energies for the optimized structures of the bare node of Ce-MOF-808 and different node-bound molecules.

\begin{tabular}{|c|c|c|c|}
\hline System & E (hartree) & H (hartree) & G (hartree) \\
\hline node & -6885.142553 & -6885.07163 & -6885.238959 \\
\hline node-noOH ${ }_{2}$ & -6808.801049 & -6808.731757 & -6808.896645 \\
\hline node-noOH & -6809.260801 & -6809.191149 & -6809.35698 \\
\hline node-noOH ${ }_{2}, \mathrm{OH}$ & -6732.927316 & -6732.860553 & -6733.02003 \\
\hline node $-\mathbf{1}$ & -7606.486092 & -7606.403271 & -7606.596062 \\
\hline node-2 & -7513.232902 & -7513.153992 & -7513.338456 \\
\hline node $-\mathbf{3}$ & -6963.596763 & -6963.523315 & -6963.696117 \\
\hline node-4 & $\begin{array}{l}-7434.77996 \\
\end{array}$ & -7434.704261 & -7434.88172 \\
\hline node $-\mathbf{5}$ & -6902.119335 & -6902.047772 & -6902.217022 \\
\hline node-6 & -7558.26861 & -7558.188621 & -7558.37483 \\
\hline node -7 & -7457.966702 & -7457.889837 & -7458.068819 \\
\hline node $-\mathbf{8}$ & -7002.840671 & -7002.766149 & -7002.940511 \\
\hline node $-\mathbf{9}$ & -7340.271429 & -7340.198934 & -7340.368924 \\
\hline node-10 & -6909.172028 & -6909.101872 & -6909.267672 \\
\hline node-11 & -7675.979377 & -7675.895588 & -7676.088838 \\
\hline node-12 & -7575.679474 & -7575.598287 & -7575.786018 \\
\hline node-13 & -7120.549363 & -7120.470797 & -7120.653348 \\
\hline node-14 & -8187.052875 & -8186.961729 & -8187.170427 \\
\hline node-15 & -7418.726543 & -7418.650604 & -7418.828206 \\
\hline node-16 & -8032.298178 & -8032.212931 & -8032.409428 \\
\hline node-17 & -7577.176992 & -7577.093913 & -7577.28645 \\
\hline node-18 & -7710.208675 & -7710.124382 & -7710.318731 \\
\hline node-19 & -7609.899091 & -7609.816621 & -7610.007944 \\
\hline node-20 & -7785.353257 & -7785.267726 & -7785.465022 \\
\hline node-21 & -7685.047479 & -7684.964275 & -7685.157235 \\
\hline node-22 & -7494.993463 & -7494.913945 & -7495.099382 \\
\hline node-23 & -7379.483113 & -7379.408588 & -7379.5831 \\
\hline node-24 & -6924.356828 & -6924.284835 & -6924.454055 \\
\hline
\end{tabular}


Table S30. The SCF energies, enthalpies, and free energies for the optimized structures of the bare node of Th-MOF-808 and different node-bound molecules.

\begin{tabular}{|c|c|c|c|}
\hline System & E (hartree) & H (hartree) & G (hartree) \\
\hline node & -6481.858265 & -6481.787327 & -6481.956213 \\
\hline node-noOH ${ }_{2}$ & -6405.519221 & -6405.449845 & -6405.616648 \\
\hline node-noOH & -6405.99 & -6405.920835 & -6406.08698 \\
\hline node-noOH ${ }_{2}, \mathrm{OH}$ & -6329.657515 & -6329.590782 & -6329.752304 \\
\hline node-1 & -7203.200037 & -7203.116937 & -7203.31208 \\
\hline node-2 & -7109.957571 & -7109.878482 & -7110.064865 \\
\hline node- $\mathbf{3}$ & -6560.313582 & -6560.239674 & -6560.415534 \\
\hline node-4 & -7031.502967 & -7031.426637 & -7031.60767 \\
\hline node $-\mathbf{5}$ & -6498.842545 & -6498.770655 & -6498.942767 \\
\hline node-6 & -7154.979318 & -7154.898892 & -7155.088051 \\
\hline node -7 & -7054.689352 & -7054.611664 & -7054.795072 \\
\hline node $-\mathbf{8}$ & -6599.558168 & -6599.483254 & -6599.660325 \\
\hline node $-\mathbf{9}$ & -6936.994914 & -6936.921461 & -6937.095868 \\
\hline node $-\mathbf{1 0}$ & -6505.890821 & -6505.820462 & -6505.988453 \\
\hline node-11 & -7272.691242 & -7272.607042 & -7272.803067 \\
\hline node-12 & -7172.397933 & -7172.316382 & -7172.506985 \\
\hline node $-\mathbf{1 3}$ & -6717.262733 & -6717.183947 & -6717.36861 \\
\hline node-14 & -7783.763905 & -7783.671944 & -7783.884975 \\
\hline node-15 & -7015.450289 & -7015.373827 & -7015.554835 \\
\hline node-16 & -7629.023583 & -7628.937927 & -7629.137167 \\
\hline node $-\mathbf{1 7}$ & -7173.900053 & -7173.81713 & -7174.011185 \\
\hline node-18 & -7306.921863 & -7306.836932 & -7307.034825 \\
\hline node $-\mathbf{1 9}$ & -7206.617486 & -7206.534871 & -7206.728317 \\
\hline node-20 & -7382.068494 & -7381.982391 & -7382.183094 \\
\hline node-21 & -7281.769597 & -7281.685918 & -7281.882051 \\
\hline node-22 & -7091.71055 & -7091.631146 & -7091.818063 \\
\hline node-23 & -6976.20666 & -6976.131446 & -6976.309673 \\
\hline node-24 & -6521.071933 & -6520.999369 & -6521.17207 \\
\hline
\end{tabular}




\section{Cartesian Coordinates}

The optimized cartesian coordinates (in $\AA$ ) for every system (molecules, nodes, and node-bound molecules) are provided in the supplementary file (Coordinates.zip).

\section{References}

1. Zhao, Y.; Truhlar, D. G. The M06 Suite of Density Functionals for Main Group Thermochemistry, Thermochemical Kinetics, Noncovalent Interactions, Excited States, and Transition Elements: Two New Functionals and Systematic Testing of Four M06-Class Functionals and 12 Other Functionals. Theor. Chem. Acc. 2008, 120, 215-241.

2. Zhao, Y.; Truhlar, D. G. Applications and validations of the Minnesota density functionals. Chem. Phys. Lett. 2011, 502, 1-13.

3. Mondloch, J. E.; Katz, M. J.; Isley III, W. C.; Ghosh, P.; Liao, P.; Bury, W.; Wagner, G. W.; Hall, M. G.; DeCoste, J. B.; Peterson, G. W.; Snurr, R. Q.; Cramer, C. J.; Hupp, J. T.; Farha, O. K. Destruction of Chemical Warfare Agents Using Metal-Organic Frameworks. Nat. Mater. 2015, 14, 512-516.

4. Islamoglu, T.; Ortuño, M. A.; Proussaloglou, E.; Howarth, A. J.; Vermeulen, N. A.; Atilgan, A.; Asiri, A. M.; Cramer, C. J.; Farha, O. K. Presence versus Proximity: The Role of Pendant Amines in the Catalytic Hydrolysis of a Nerve Agent Simulant. Angew. Chem., Int. Ed. 2018, 57, 1949-1953.

5. Momeni, M. R.; Cramer, C. J. Dual Role of Water in Heterogeneous Catalytic Hydrolysis of Sarin by Zirconium-Based Metal-Organic Frameworks. ACS Appl. Mater. Interfaces 2018, 10, $18435-18439$.

6. Momeni, M. R.; Cramer, C. J. Structural Characterization of Pristine and Defective $\left[\mathrm{Zr}_{12}\left(\mu_{3}-\right.\right.$ $\left.\mathrm{O})_{8}\left(\mu_{3}-\mathrm{OH}\right)_{8}\left(\mu_{2}-\mathrm{OH}\right)_{6}\right]^{18+}$ Double-Node Metal-Organic Framework and Predicted Applications for Single-Site Catalytic Hydrolysis of Sarin. Chem. Mater. 2018, 30, 4432-4439.

7. Becke, A. D. Density-Functional Thermochemistry. III. The Role of Exact Exchange. J. Chem. Phys. 1993, 98, 5648.

8. Lee, C.; Yang, W.; Parr, R. G. Development of the Colle-Salvetti Correlation-Energy Formula into a Functional of the Electron Density. Phys. Rev. B: Condens. Matter Mater. Phys. 1988, 37, 785-789.

9. Grimme, S.; Antony, J.; Ehrlich, S.; Krieg, H. A Consistent and Accurate Ab Initio Parametrization of Density Functional Dispersion Correction (DFT-D) for the 94 Elements HPu. J. Chem. Phys. 2010, 132, 154104.

10. Becke, A. D.; Johnson, E. R. A Density-Functional Model of the Dispersion Interaction. $J$. Chem. Phys. 2005, 123, 154101.

11. Grimme, S.; Ehrlich, S.; Goerigk, L. Effect of the Damping Function in Dispersion Corrected Density Functional Theory. J. Comput. Chem. 2011, 32, 1456-1465.

12. Chai, J.-D.; Head-Gordon, M. Long-range corrected hybrid density functionals with damped atom-atom dispersion corrections. Phys. Chem. Chem. Phys. 2008, 10, 6615-6620.

13. Weigend, F.; Ahlrichs, R. Balanced Basis Sets of Split Valence, Triple Zeta Valence and Quadruple Zeta Valence Quality for H to Rn: Design and Assessment of Accuracy. Phys. Chem. Chem. Phys. 2005, 7, 3297-3305. 
14. Witte, J.; Mardirossian, N.; Neaton, J. B.; Head-Gordon, M. Assessing DFT-D3 Damping Functions Across Widely Used Density Functionals: Can We Do Better? J. Chem. Theory Comput. 2017, 13, 2043-2052.

15. Koopmans, T. Über Die Zuordnung von Wellenfunktionen Und Eigenwerten Zu Den Einzelnen Elektronen Eines Atoms. Physica 1934, 1, 104-113.

16. Parr, R. G.; Chattaraj, P. K. Principle of Maximum Hardness. J. Am. Chem. Soc. 1991, 113, 1854-1855.

17. Talete. Dragon Version 6.0. 2010, http://www.talete.mi.it/

18. Todeschini, R.; Vighi, M.; Finizio, A.; Gramatica, P. 3D Modeling and Prediction by WHIM Descriptors. Part 8. Toxicity and Physico-Chemical Properties of Environmental Priority Chemicals by 2D-TI and 3D-WHIM Descriptors. SAR \& QSAR Environ. Res. 1997, 7, 173193.

19. Mitra, I.; Saha, A.; Roy, K. Exploring Quantitative Structure-Activity Relationship (QSAR) Studies of Antioxidant Phenolic Compounds Obtained from Traditional Chinese Medicinal Plants. Mol. Simul. 2010, 36, 1067-1079.

\section{Full Citation of Reference 39 in the Main Text}

Gaussian 09, Revision D.01, M. J. Frisch, G. W. Trucks, H. B. Schlegel, G. E. Scuseria, M. A. Robb, J. R. Cheeseman, G. Scalmani, V. Barone, B. Mennucci, G. A. Petersson, H. Nakatsuji, M. Caricato, X. Li, H. P. Hratchian, A. F. Izmaylov, J. Bloino, G. Zheng, J. L. Sonnenberg, M. Hada, M. Ehara, K. Toyota, R. Fukuda, J. Hasegawa, M. Ishida, T. Nakajima, Y. Honda, O. Kitao, H. Nakai, T. Vreven, J. A. Montgomery, Jr., J. E. Peralta, F. Ogliaro, M. Bearpark, J. J. Heyd, E. Brothers, K. N. Kudin, V. N. Staroverov, T. Keith, R. Kobayashi, J. Normand, K. Raghavachari, A. Rendell, J. C. Burant, S. S. Iyengar, J. Tomasi, M. Cossi, N. Rega, J. M. Millam, M. Klene, J. E. Knox, J. B. Cross, V. Bakken, C. Adamo, J. Jaramillo, R. Gomperts, R. E. Stratmann, O. Yazyev, A. J. Austin, R. Cammi, C. Pomelli, J. W. Ochterski, R. L. Martin, K. Morokuma, V. G. Zakrzewski, G. A. Voth, P. Salvador, J. J. Dannenberg, S. Dapprich, A. D. Daniels, O. Farkas, J. B. Foresman, J. V. Ortiz, J. Cioslowski, and D. J. Fox, Gaussian, Inc., Wallingford CT, 2013. 